\title{
Why Are Esters and Amides Weaker Carbon Acids than Ketones and Acid Fluroides? Contributions by Resonance and Inductive Effects
}

\author{
Alexandra Fersner, ${ }^{\dagger}$ Joel M. Karty, ${ }^{*}{ }^{\dagger}$ and Yirong Mo*, ${ }^{*}$
}

\section{Table of contents.}

1. Cartesian coordinates, absolute energies, zero-point energy corrections, absolute enthalpies, and number of imaginary frequencies for acetone (1), acetone enolate anion, and their $n=1-4$

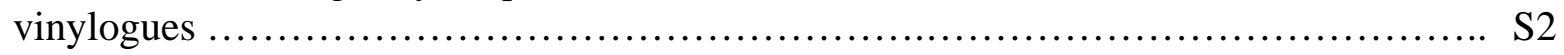

2. Cartesian coordinates, absolute energies, zero-point energy corrections, absolute enthalpies, and number of imaginary frequencies for acetamide (2), acetamide enolate anion, and their $n=1-4$ vinylogues

3. Cartesian coordinates, absolute energies, zero-point energy corrections, enthalpies, and number of imaginary frequencies for acetic acid (3), acetic acid enolate anion, and their $n=1-4$ vinylogues.

4. Cartesian coordinates, absolute energies, zero-point energy corrections, absolute enthalpies, and number of imaginary frequencies for acetyl fluoride (4), acetyl fluoride enolate anion, and their $n=1-4$ vinylogues

5. Cartesian coordinates, absolute energies, zero-point energy corrections, absolute enthalpies, and number of imaginary frequencies for methylpropane (5), the methylpropyl anion, and their $n=1-4$ vinylogues S55

6. Cartesian coordinates and absolute energies of the delocalized and localized structures of acetone (1), acetone enolate anion, acetamide (2), acetamide enolate anion, acetic acid (3), acetic acid enolate anion, acetyl fluoride (4), acetyl fluoride enolate anion, methylpropane (5), and the methylpropyl anion, derived from the BLW method.

S63 
1. Cartesian coordinates, absolute energies, zero-point energy corrections, absolute enthalpies, and number of imaginary frequencies for acetone (1), acetone enolate anion, and their $n=1-4$ vinylogues

Table S1. Acetone neutral $n=0$.

\begin{tabular}{|c|c|c|c|c|c|}
\hline \multirow{2}{*}{$\begin{array}{l}\text { Center } \\
\text { Number }\end{array}$} & \multirow{2}{*}{\multicolumn{2}{|c|}{$\begin{array}{l}\text { Atomic } \\
\text { Number }\end{array}$}} & Atomic & \multicolumn{2}{|c|}{ Coordinates (Angstroms) } \\
\hline & & & Type & $\begin{array}{ll}X & Y\end{array}$ & Z \\
\hline 1 & 6 & 0 & -0.000002 & 0.182370 & -0.000022 \\
\hline 2 & 6 & 0 & -1.293612 & -0.613917 & -0.001920 \\
\hline 3 & 1 & 0 & -1.294982 & -1.354555 & -0.811628 \\
\hline 4 & 1 & 0 & -1.389461 & -1.171378 & 0.939487 \\
\hline 5 & 1 & 0 & -2.148278 & 0.057373 & -0.111439 \\
\hline 6 & 6 & 0 & 1.293630 & -0.613929 & 0.001921 \\
\hline 7 & 1 & 0 & 1.295337 & -1.354005 & 0.812140 \\
\hline 8 & 1 & 0 & 1.389087 & -1.171995 & -0.939151 \\
\hline 9 & 1 & 0 & 2.148241 & 0.057562 & 0.110643 \\
\hline 10 & 8 & 0 & -0.000005 & 1.401232 & 0.000010 \\
\hline
\end{tabular}

$E=-193.16626074$ a.u.

ZPE correction $=0.083930$ a.u.

$\mathrm{H}=-193.07602$ a.u.

0 imaginary frequencies

Table S2. Acetone anion $\mathrm{n}=0$.

\begin{tabular}{|c|c|c|c|c|c|}
\hline \multirow{2}{*}{\multicolumn{2}{|c|}{$\begin{array}{l}\text { Center } \\
\text { Number }\end{array}$}} & \multirow{2}{*}{$\begin{array}{l}\text { Atomic } \\
\text { Number }\end{array}$} & \multirow{2}{*}{$\begin{array}{l}\text { Atomic } \\
\text { Type }\end{array}$} & \multicolumn{2}{|c|}{ Coordinates (Angstroms) } \\
\hline & & & & $\begin{array}{ll}X & Y\end{array}$ & Z \\
\hline 1 & 6 & 0 & 1.294970 & -0.483598 & -0.000007 \\
\hline 2 & 1 & 0 & 1.840686 & -0.121360 & 0.883571 \\
\hline 3 & 1 & 0 & 1.841273 & -0.119561 & -0.882525 \\
\hline 4 & 1 & 0 & 1.313065 & -1.583231 & -0.001143 \\
\hline 5 & 6 & 0 & -0.133941 & 0.110530 & -0.000007 \\
\hline 6 & 8 & 0 & -0.194570 & 1.387379 & 0.000016 \\
\hline 7 & 6 & 0 & -1.192488 & -0.789864 & 0.000013 \\
\hline 8 & 1 & 0 & -2.221041 & -0.430806 & -0.000193 \\
\hline 9 & 1 & 0 & -1.028668 & -1.866485 & 0.000166 \\
\hline
\end{tabular}

$E=-192.56791090$ a.u.

ZPE correction $=0.070404$ a.u.

$\mathrm{H}=-192.491898$ a.u.

0 imaginary frequencies 
Table S3. Acetone neutral $n=1$ parallel.

\begin{tabular}{|c|c|c|c|c|c|}
\hline \multirow{2}{*}{\multicolumn{2}{|c|}{$\begin{array}{l}\text { Center } \\
\text { Number }\end{array}$}} & \multirow{2}{*}{$\begin{array}{l}\text { Atomic } \\
\text { Number }\end{array}$} & Atomic & \multicolumn{2}{|c|}{ Coordinates (Angstroms } \\
\hline & & & r Type & $X \quad Y$ & Z \\
\hline 1 & 6 & 0 & 1.572429 & 1.254040 & 0.000016 \\
\hline 2 & 1 & 0 & 2.663248 & 1.303282 & -0.002535 \\
\hline 3 & 1 & 0 & 1.190218 & 1.775372 & 0.886263 \\
\hline 4 & 1 & 0 & 1.185746 & 1.777792 & -0.882792 \\
\hline 5 & 6 & 0 & 1.147267 & -0.206598 & -0.000126 \\
\hline 6 & 8 & 0 & 1.986420 & -1.101122 & 0.000029 \\
\hline 7 & 6 & 0 & -0.294132 & -0.556899 & -0.000072 \\
\hline 8 & 1 & 0 & -0.487859 & -1.628830 & 0.000127 \\
\hline 9 & 6 & 0 & -1.311308 & 0.321494 & -0.000125 \\
\hline 10 & 1 & 0 & -1.093445 & 1.389983 & -0.000353 \\
\hline 11 & 6 & 0 & -2.763979 & -0.047840 & 0.000094 \\
\hline 12 & 1 & 0 & -3.271656 & 0.369439 & 0.880536 \\
\hline 13 & 1 & 0 & -3.271883 & 0.369743 & -0.880115 \\
\hline 14 & 1 & 0 & -2.907386 & -1.132987 & -0.000083 \\
\hline
\end{tabular}

$E=-270.56979485$ a.u.

ZPE correction $=0.117998 \mathrm{a} . \mathrm{u}$.

$\mathrm{H}=-270.443554$ a.u.

0 imaginary frequencies

Table S4. Acetone anion $n=1$ parallel.

\begin{tabular}{|c|c|c|c|c|c|}
\hline \multirow{2}{*}{$\begin{array}{l}\text { Center } \\
\text { Number }\end{array}$} & \multirow{2}{*}{\multicolumn{2}{|c|}{$\begin{array}{l}\text { Atomic } \\
\text { Number }\end{array}$}} & \multirow{2}{*}{$\begin{array}{l}\text { Atomic } \\
\text { Type }\end{array}$} & \multicolumn{2}{|c|}{ Coordinates (Angstroms) } \\
\hline & & & & $X \quad Y$ & Z \\
\hline 1 & 6 & 0 & -1.040067 & -0.216523 & -0.000185 \\
\hline 2 & 8 & 0 & -1.996858 & -1.050509 & 0.000209 \\
\hline 3 & 6 & 0 & -1.469136 & 1.263781 & -0.000100 \\
\hline 4 & 1 & 0 & -0.648328 & 1.989812 & -0.002986 \\
\hline 5 & 1 & 0 & -2.100096 & 1.445947 & -0.880723 \\
\hline 6 & 1 & 0 & -2.095167 & 1.447422 & 0.883740 \\
\hline 7 & 6 & 0 & 0.323642 & -0.573998 & -0.000151 \\
\hline 8 & 1 & 0 & 0.511385 & -1.650127 & -0.000203 \\
\hline 9 & 6 & 0 & 1.459212 & 0.286285 & 0.000017 \\
\hline 10 & 1 & 0 & 1.256743 & 1.360860 & 0.000727 \\
\hline 11 & 6 & 0 & 2.787962 & -0.057959 & 0.000137 \\
\hline 12 & 1 & 0 & 3.111511 & -1.098964 & -0.000606 \\
\hline 13 & 1 & 0 & 3.569142 & 0.699613 & 0.000073 \\
\hline \multicolumn{6}{|c|}{$\begin{array}{l}E=-269.99092729 \text { a.u. } \\
\text { ZPE correction }=0.103931 \text { a.u. } \\
H=-269.879183 \text { a.u. } \\
0 \text { imaginary frequencies }\end{array}$} \\
\hline
\end{tabular}


Table S5. Acetone neutral $n=1$ perpendicular.

\begin{tabular}{|c|c|c|c|c|c|}
\hline \multirow{2}{*}{$\begin{array}{l}\text { Center } \\
\text { Number }\end{array}$} & \multicolumn{2}{|c|}{ Atomic } & Atomic & \multicolumn{2}{|c|}{ Coordinates (Angstroms } \\
\hline & & Number & Type & $\begin{array}{ll}X & Y\end{array}$ & Z \\
\hline 1 & 6 & 0 & -1.131635 & -0.193887 & 0.074198 \\
\hline 2 & 8 & 0 & -1.648884 & -1.281344 & -0.118788 \\
\hline 3 & 6 & 0 & -1.908967 & 1.101083 & -0.058144 \\
\hline 4 & 1 & 0 & -1.473045 & 1.714237 & -0.857723 \\
\hline 5 & 1 & 0 & -2.959432 & 0.894681 & -0.278415 \\
\hline 6 & 1 & 0 & -1.826126 & 1.689054 & 0.865273 \\
\hline 7 & 6 & 0 & 0.313913 & -0.080415 & 0.485867 \\
\hline 8 & 1 & 0 & 0.497279 & -0.104933 & 1.562254 \\
\hline 9 & 6 & 0 & 1.331827 & 0.022438 & -0.374494 \\
\hline 10 & 1 & 0 & 1.116872 & 0.040198 & -1.445041 \\
\hline 11 & 6 & 0 & 2.783220 & 0.096317 & 0.007432 \\
\hline 12 & 1 & 0 & 2.915705 & 0.071867 & 1.094541 \\
\hline 13 & 1 & 0 & 3.343719 & -0.743647 & -0.424624 \\
\hline 14 & 1 & 0 & 3.245948 & 1.016085 & -0.375110 \\
\hline
\end{tabular}

$E=-270.55881763$ a.u.

ZPE correction $=0.117035 \mathrm{a} . \mathrm{u}$.

$\mathrm{H}=-270.434116$ a.u.

1 imaginary frequency

Table S6. Acetone anion $n=1$ perpendicular.

\begin{tabular}{|c|c|c|c|c|c|}
\hline \multirow{2}{*}{$\begin{array}{l}\text { Center } \\
\text { Number }\end{array}$} & \multicolumn{2}{|c|}{ Atomic } & Atomic & \multicolumn{2}{|c|}{ Coordinates (Angstroms) } \\
\hline & & Number & Type & $x$ & Z \\
\hline 1 & 6 & 0 & -1.041085 & -0.204614 & 0.088993 \\
\hline 2 & 8 & 0 & -1.648789 & -1.248253 & -0.164984 \\
\hline 3 & 6 & 0 & -1.811222 & 1.117450 & -0.082774 \\
\hline 4 & 1 & 0 & -1.275716 & 1.754377 & -0.798844 \\
\hline 5 & 1 & 0 & -2.844765 & 0.954424 & -0.420698 \\
\hline 6 & 1 & 0 & -1.808228 & 1.663461 & 0.870002 \\
\hline 7 & 6 & 0 & 0.358060 & -0.104148 & 0.566915 \\
\hline 8 & 1 & 0 & 0.491536 & -0.115151 & 1.651497 \\
\hline 9 & 6 & 0 & 1.453167 & -0.000928 & -0.299271 \\
\hline 10 & 1 & 0 & 1.185138 & -0.003722 & -1.365637 \\
\hline 11 & 6 & 0 & 2.821258 & 0.104684 & -0.055989 \\
\hline 12 & 1 & 0 & 3.230794 & 0.093785 & 0.954659 \\
\hline 13 & 1 & 0 & 3.530489 & 0.164184 & -0.878348 \\
\hline \multicolumn{6}{|c|}{$E=-269.9414498$ a.u. } \\
\hline \multicolumn{6}{|c|}{ ZPE correction = 0.100783 a.u. } \\
\hline \multicolumn{6}{|c|}{$H=-269.832779$ a.u. } \\
\hline \multicolumn{6}{|c|}{1 imaginary frequency } \\
\hline
\end{tabular}


Table S7. Acetone neutral $n=2$ parallel.

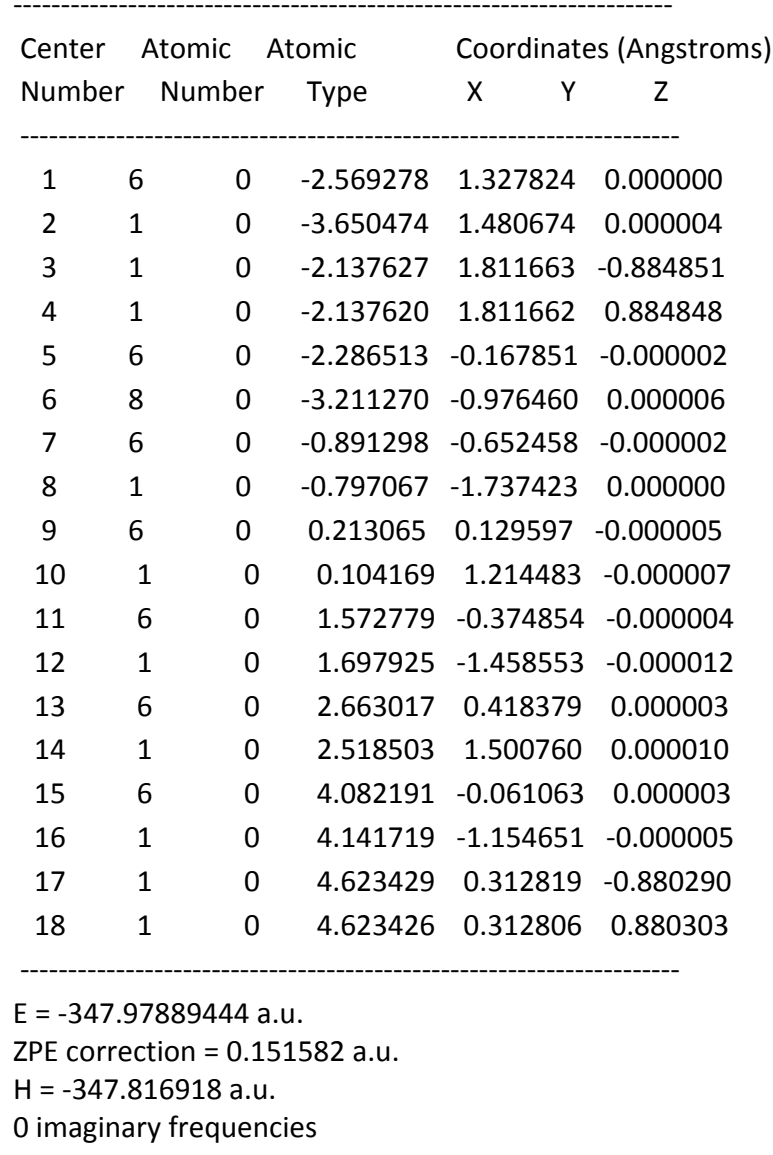


Table S8. Acetone anion $\mathrm{n}=2$ parallel

\begin{tabular}{|c|c|c|c|c|c|}
\hline \multirow{2}{*}{$\begin{array}{l}\text { Center } \\
\text { Number }\end{array}$} & \multirow{2}{*}{\multicolumn{2}{|c|}{$\begin{array}{l}\text { Atomic } \\
\text { Number }\end{array}$}} & Atomic & \multicolumn{2}{|c|}{ Coordinates (Angstroms) } \\
\hline & & & Type & $X \quad Y$ & Z \\
\hline 1 & 6 & 0 & 2.190858 & -0.165300 & -0.000038 \\
\hline 2 & 8 & 0 & 3.217221 & -0.899829 & -0.000025 \\
\hline 3 & 6 & 0 & 2.458154 & 1.349360 & -0.000075 \\
\hline 4 & 1 & 0 & 1.563223 & 1.980170 & -0.000576 \\
\hline 5 & 1 & 0 & 3.062014 & 1.598438 & 0.882440 \\
\hline 6 & 1 & 0 & 3.062865 & 1.598173 & -0.882071 \\
\hline 7 & 6 & 0 & 0.865196 & -0.672873 & -0.000018 \\
\hline 8 & 1 & 0 & 0.801203 & -1.762495 & -0.000054 \\
\hline 9 & 6 & 0 & -0.340625 & 0.055703 & 0.000090 \\
\hline 10 & 1 & 0 & -0.267161 & 1.146593 & 0.000172 \\
\hline 11 & 6 & 0 & -1.635156 & -0.445554 & 0.000174 \\
\hline 12 & 1 & 0 & -1.779795 & -1.528643 & 0.000128 \\
\hline 13 & 6 & 0 & -2.800919 & 0.381061 & 0.000243 \\
\hline 14 & 1 & 0 & -2.601762 & 1.458765 & 0.000127 \\
\hline 15 & 6 & 0 & -4.113529 & 0.001201 & -0.000259 \\
\hline 16 & 1 & 0 & -4.405833 & -1.048476 & -0.000406 \\
\hline 17 & 1 & 0 & -4.916392 & 0.734523 & -0.000264 \\
\hline
\end{tabular}

$E=-347.41329720$ a.u.

ZPE correction $=0.137352$ a.u.

$\mathrm{H}=-347.26591$ a.u.

0 imaginary frequencies

Table S9. Acetone neutral $n=2$ perpendicular.

\begin{tabular}{|c|c|c|c|c|c|}
\hline \multirow{2}{*}{$\begin{array}{l}\text { Center } \\
\text { Number }\end{array}$} & \multirow{2}{*}{\multicolumn{2}{|c|}{$\begin{array}{l}\text { Atomic } \\
\text { Number }\end{array}$}} & \multirow{2}{*}{$\begin{array}{l}\text { Atomic } \\
\text { Type }\end{array}$} & \multicolumn{2}{|c|}{ Coordinates (Angstroms) } \\
\hline & & & & $X \quad Y$ & Z \\
\hline 1 & 6 & 0 & -2.274246 & -0.175762 & 0.041887 \\
\hline 2 & 8 & 0 & -2.811838 & -1.228966 & -0.257315 \\
\hline 3 & 6 & 0 & -2.988098 & 1.155870 & -0.085222 \\
\hline 4 & 1 & 0 & -2.454845 & 1.796647 & -0.799573 \\
\hline 5 & 1 & 0 & -4.018706 & 1.005603 & -0.417045 \\
\hline 6 & 1 & 0 & -2.975781 & 1.685025 & 0.876622 \\
\hline 7 & 6 & 0 & -0.870365 & -0.144810 & 0.584723 \\
\hline 8 & 1 & 0 & -0.782806 & -0.231409 & 1.669377 \\
\hline 9 & 6 & 0 & 0.229268 & -0.038378 & -0.181843 \\
\hline 10 & 1 & 0 & 0.115926 & 0.043168 & -1.264584 \\
\hline 11 & 6 & 0 & 1.594327 & -0.037400 & 0.326069 \\
\hline 12 & 1 & 0 & 1.714700 & -0.127127 & 1.407282 \\
\hline 13 & 6 & 0 & 2.687320 & 0.063162 & -0.452643 \\
\hline 14 & 1 & 0 & 2.551341 & 0.148788 & -1.532736 \\
\hline 15 & 6 & 0 & 4.103923 & 0.063633 & 0.039826 \\
\hline 16 & 1 & 0 & 4.624453 & 0.986558 & -0.251789 \\
\hline 17 & 1 & 0 & 4.153313 & -0.025225 & 1.130724 \\
\hline 18 & 1 & 0 & 4.674329 & -0.768181 & -0.396533 \\
\hline $\begin{array}{l}H=-34 \\
1 \text { imagir }\end{array}$ & & $\begin{array}{l}06129 \text { a.u. } \\
\text { y frequenc }\end{array}$ & 381 a.u. & & \\
\hline
\end{tabular}


Table S10. Acetone anion $\mathrm{n}=2$ perpendicular.

\begin{tabular}{|c|c|c|c|c|c|}
\hline \multirow{2}{*}{$\begin{array}{l}\text { Center } \\
\text { Number }\end{array}$} & \multirow{2}{*}{\multicolumn{2}{|c|}{$\begin{array}{l}\text { Atomic } \\
\text { Number }\end{array}$}} & \multirow{2}{*}{$\begin{array}{l}\text { Atomic } \\
\text { Type }\end{array}$} & \multicolumn{2}{|c|}{ Coordinates (Angstroms) } \\
\hline & & & & $X \quad Y$ & Z \\
\hline 1 & 6 & 0 & -2.186611 & -0.182657 & 0.019246 \\
\hline 2 & 8 & 0 & -2.785750 & -1.167808 & -0.411153 \\
\hline 3 & 6 & 0 & -2.867340 & 1.186900 & -0.094075 \\
\hline 4 & 1 & 0 & -2.939437 & 1.643508 & 0.901892 \\
\hline 5 & 1 & 0 & -2.233703 & 1.856209 & -0.690566 \\
\hline 6 & 1 & 0 & -3.864028 & 1.108493 & -0.547075 \\
\hline 7 & 6 & 0 & -0.849517 & -0.210098 & 0.669906 \\
\hline 8 & 1 & 0 & -0.854268 & -0.340991 & 1.754328 \\
\hline 9 & 6 & 0 & 0.338543 & -0.080578 & -0.028779 \\
\hline 10 & 1 & 0 & 0.237733 & 0.041327 & -1.115519 \\
\hline 11 & 6 & 0 & 1.667982 & -0.081065 & 0.437443 \\
\hline 12 & 1 & 0 & 1.843304 & -0.209245 & 1.509248 \\
\hline 13 & 6 & 0 & 2.791538 & 0.074483 & -0.412456 \\
\hline 14 & 1 & 0 & 2.543757 & 0.192759 & -1.475379 \\
\hline 15 & 6 & 0 & 4.134161 & 0.099034 & -0.105701 \\
\hline 16 & 1 & 0 & 4.491997 & -0.011544 & 0.918125 \\
\hline 17 & 1 & 0 & 4.888110 & 0.225828 & -0.879330 \\
\hline $\begin{array}{l}E=-347 . \\
Z P E \text { corr } \\
H=-347 \\
1 \text { imagin }\end{array}$ & rect & $\begin{array}{l}2052 \text { a.u. } \\
\text { tion }=0.13 \\
27517 \text { a.u. } \\
\text { y frequen }\end{array}$ & 4762 a.u. & & \\
\hline
\end{tabular}


Table S11. Acetone neutral $n=3$ parallel.

\begin{tabular}{|c|c|c|c|c|c|}
\hline \multirow{2}{*}{$\begin{array}{l}\text { Center } \\
\text { Number }\end{array}$} & \multirow{2}{*}{\multicolumn{2}{|c|}{$\begin{array}{l}\text { Atomic } \\
\text { Number }\end{array}$}} & Atomic & \multicolumn{2}{|c|}{ Coordinates (Angstroms) } \\
\hline & & & Type & $X \quad Y$ & Z \\
\hline 1 & 6 & 0 & 3.672508 & 1.384371 & 0.000004 \\
\hline 2 & 1 & 0 & 4.745624 & 1.586347 & -0.000001 \\
\hline 3 & 1 & 0 & 3.219513 & 1.848455 & 0.884800 \\
\hline 4 & 1 & 0 & 3.219504 & 1.848458 & -0.884785 \\
\hline 5 & 6 & 0 & 3.458316 & -0.123012 & 0.000002 \\
\hline 6 & 8 & 0 & 4.420427 & -0.888115 & -0.000004 \\
\hline 7 & 6 & 0 & 2.088746 & -0.670041 & 0.000005 \\
\hline 8 & 1 & 0 & 2.043208 & -1.758046 & 0.000016 \\
\hline 9 & 6 & 0 & 0.946964 & 0.062160 & -0.000005 \\
\hline 10 & 1 & 0 & 1.008795 & 1.150729 & -0.000016 \\
\hline 11 & 6 & 0 & -0.381704 & -0.500071 & -0.000002 \\
\hline 12 & 1 & 0 & -0.462045 & -1.587614 & 0.000002 \\
\hline 13 & 6 & 0 & -1.515020 & 0.248939 & -0.000004 \\
\hline 14 & 1 & 0 & -1.424078 & 1.336804 & -0.000008 \\
\hline 15 & 6 & 0 & -2.857357 & -0.291166 & -0.000001 \\
\hline 16 & 1 & 0 & -2.951582 & -1.378463 & 0.000001 \\
\hline 17 & 6 & 0 & -3.974774 & 0.465445 & 0.000000 \\
\hline 18 & 1 & 0 & -3.866920 & 1.551968 & -0.000002 \\
\hline 19 & 6 & 0 & -5.376688 & -0.062836 & 0.000003 \\
\hline 20 & 1 & 0 & -5.397953 & -1.158012 & 0.000005 \\
\hline 21 & 1 & 0 & -5.931716 & 0.290782 & 0.880270 \\
\hline 22 & 1 & 0 & -5.931719 & 0.290779 & -0.880263 \\
\hline $\begin{array}{l}\text { ZPE corre } \\
\mathrm{H}=-425 . \\
0 \text { imagin }\end{array}$ & & $\begin{array}{l}\text { ion }=0.18 \\
1216 \text { a.u } \\
\text { frequen }\end{array}$ & 36 a.u. & & \\
\hline
\end{tabular}


Table S12. Acetone anion $n=3$ parallel.

\begin{tabular}{|c|c|c|c|c|c|}
\hline \multirow{2}{*}{$\begin{array}{l}\text { Center } \\
\text { Number }\end{array}$} & \multicolumn{2}{|c|}{ Atomic } & Atomic & \multicolumn{2}{|c|}{ Coordinates (Angstroms) } \\
\hline & & Number & Type & $\begin{array}{ll}X & Y\end{array}$ & Z \\
\hline 1 & 6 & 0 & -3.366796 & -0.090503 & -0.000030 \\
\hline 2 & 8 & 0 & -4.425906 & -0.768326 & 0.000048 \\
\hline 3 & 6 & 0 & -3.542622 & 1.435380 & 0.000025 \\
\hline 4 & 1 & 0 & -2.611160 & 2.009877 & -0.000986 \\
\hline 5 & 1 & 0 & -4.131148 & 1.719699 & -0.881673 \\
\hline 6 & 1 & 0 & -4.129303 & 1.719913 & 0.882894 \\
\hline 7 & 6 & 0 & -2.068736 & -0.681097 & -0.000053 \\
\hline 8 & 1 & 0 & -2.073822 & -1.772140 & -0.000030 \\
\hline 9 & 6 & 0 & -0.830030 & -0.028207 & -0.000049 \\
\hline 10 & 1 & 0 & -0.832444 & 1.064382 & -0.000054 \\
\hline 11 & 6 & 0 & 0.435541 & -0.618225 & -0.000012 \\
\hline 12 & 1 & 0 & 0.500970 & -1.708475 & 0.000025 \\
\hline 13 & 6 & 0 & 1.641907 & 0.119499 & -0.000016 \\
\hline 14 & 1 & 0 & 1.532223 & 1.210020 & -0.000064 \\
\hline 15 & 6 & 0 & 2.935914 & -0.366355 & 0.000008 \\
\hline 16 & 1 & 0 & 3.091370 & -1.447743 & 0.000042 \\
\hline 17 & 6 & 0 & 4.098609 & 0.470184 & -0.000015 \\
\hline 18 & 1 & 0 & 3.896512 & 1.546332 & -0.000105 \\
\hline 19 & 6 & 0 & 5.406032 & 0.085328 & 0.000046 \\
\hline 20 & 1 & 0 & 5.692722 & -0.965610 & 0.000126 \\
\hline 21 & 1 & 0 & 6.212414 & 0.814330 & 0.000008 \\
\hline $\begin{array}{l}\text { ZPE corre } \\
\mathrm{H}=-424 \text {. } \\
0 \text { imagin }\end{array}$ & & $\begin{array}{l}250490 \\
t 9495 \\
\text { tion }\end{array}$ & 835 a.u. & & \\
\hline
\end{tabular}


Table S13. Acetone neutral $n=3$ perpendicular.

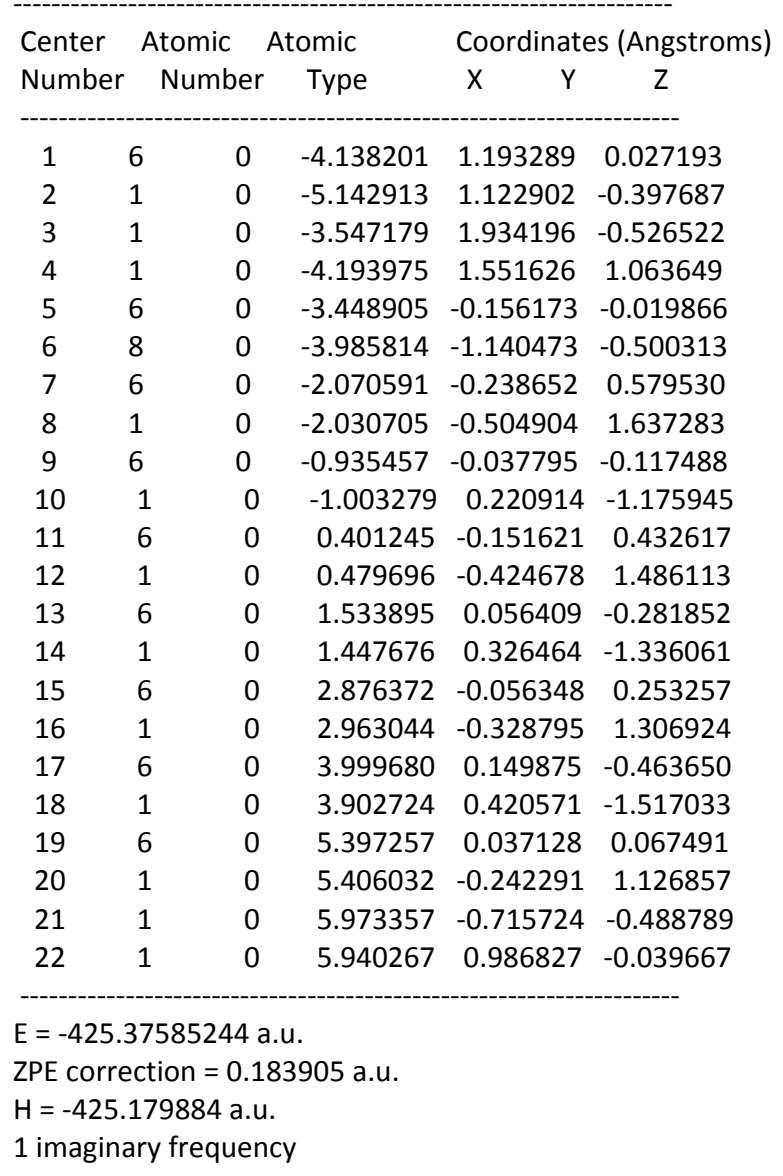


Table S14. Acetone anion $\mathrm{n}=3$ perpendicular.

\begin{tabular}{|c|c|c|c|c|c|}
\hline \multirow{2}{*}{$\begin{array}{l}\text { Center } \\
\text { Number }\end{array}$} & \multirow{2}{*}{\multicolumn{2}{|c|}{$\begin{array}{l}\text { Atomic } \\
\text { Number }\end{array}$}} & \multirow{2}{*}{$\begin{array}{l}\text { Atomic } \\
\text { Type }\end{array}$} & \multicolumn{2}{|c|}{ Coordinates (Angstroms) } \\
\hline & & & & $X \quad Y$ & Z \\
\hline 1 & 6 & 0 & 3.357269 & -0.090198 & 0.134986 \\
\hline 2 & 8 & 0 & 3.942667 & -0.655877 & 1.055199 \\
\hline 3 & 6 & 0 & 3.990966 & 1.154308 & -0.490192 \\
\hline 4 & 1 & 0 & 4.114816 & 1.001690 & -1.570505 \\
\hline 5 & 1 & 0 & 3.306139 & 2.005023 & -0.378797 \\
\hline 6 & 1 & 0 & 4.958033 & 1.386342 & -0.028390 \\
\hline 7 & 6 & 0 & 2.063167 & -0.543498 & -0.448931 \\
\hline 8 & 1 & 0 & 2.141442 & -1.241499 & -1.285195 \\
\hline 9 & 6 & 0 & 0.835332 & -0.128415 & 0.012514 \\
\hline 10 & 1 & 0 & 0.846506 & 0.569496 & 0.858527 \\
\hline 11 & 6 & 0 & -0.457178 & -0.477206 & -0.453131 \\
\hline 12 & 1 & 0 & -0.529854 & -1.178067 & -1.288521 \\
\hline 13 & 6 & 0 & -1.641195 & 0.031065 & 0.098574 \\
\hline 14 & 1 & 0 & -1.510816 & 0.727840 & 0.936229 \\
\hline 15 & 6 & 0 & -2.959947 & -0.226187 & -0.271821 \\
\hline 16 & 1 & 0 & -3.145565 & -0.914424 & -1.100664 \\
\hline 17 & 6 & 0 & -4.089427 & 0.361231 & 0.368841 \\
\hline 18 & 1 & 0 & -3.847016 & 1.042492 & 1.192872 \\
\hline 19 & 6 & 0 & -5.420200 & 0.188295 & 0.093118 \\
\hline 20 & 1 & 0 & -5.758819 & -0.469758 & -0.706802 \\
\hline 21 & 1 & 0 & -6.188922 & 0.701512 & 0.665894 \\
\hline $\begin{array}{l}E=-424 . \\
Z P E \text { corre } \\
H=-424 . \\
1 \text { imagin }\end{array}$ & $\begin{array}{l}.796 \\
\text { ecti } \\
.61 \\
\text { lary }\end{array}$ & $\begin{array}{l}65073 \text { a.u } \\
\text { tion }=0.16 \\
\text { L } 6151 \text { a.u. } \\
\text { y frequen }\end{array}$ & 3488 a.u. & & \\
\hline
\end{tabular}


Table S15. Acetone neutral $n=4$ parallel.

\begin{tabular}{|c|c|c|c|c|c|}
\hline \multirow{2}{*}{$\begin{array}{l}\text { Center } \\
\text { Number }\end{array}$} & \multirow{2}{*}{\multicolumn{2}{|c|}{$\begin{array}{l}\text { Atomic } \\
\text { Number }\end{array}$}} & tomic & \multicolumn{2}{|c|}{ Coordinates (Angstroms) } \\
\hline & & & Type & $X \quad Y$ & Z \\
\hline 1 & 6 & 0 & 4.819937 & 1.436269 & 0.000015 \\
\hline 2 & 1 & 0 & 5.886880 & 1.668817 & 0.000003 \\
\hline 3 & 1 & 0 & 4.353992 & 1.887429 & 0.884791 \\
\hline 4 & 1 & 0 & 4.353965 & 1.887454 & -0.884733 \\
\hline 5 & 6 & 0 & 4.648960 & -0.076901 & -0.000004 \\
\hline 6 & 8 & 0 & 5.633318 & -0.813854 & -0.000032 \\
\hline 7 & 6 & 0 & 3.296492 & -0.662283 & 0.000002 \\
\hline 8 & 1 & 0 & 3.281297 & -1.751133 & 0.000008 \\
\hline 9 & 6 & 0 & 2.133119 & 0.037778 & 0.000004 \\
\hline 10 & 1 & 0 & 2.165058 & 1.127641 & -0.000001 \\
\hline 11 & 6 & 0 & 0.823148 & -0.560220 & 0.000014 \\
\hline 12 & 1 & 0 & 0.771788 & -1.649475 & 0.000031 \\
\hline 13 & 6 & 0 & -0.333737 & 0.158695 & 0.000003 \\
\hline 14 & 1 & 0 & -0.270259 & 1.248557 & -0.000014 \\
\hline 15 & 6 & 0 & -1.653160 & -0.414094 & 0.000013 \\
\hline 16 & 1 & 0 & -1.722711 & -1.502879 & 0.000036 \\
\hline 17 & 6 & 0 & -2.799133 & 0.318475 & -0.000003 \\
\hline 18 & 1 & 0 & -2.724479 & 1.407490 & -0.000025 \\
\hline 19 & 6 & 0 & -4.131577 & -0.243099 & 0.000008 \\
\hline 20 & 1 & 0 & -4.207097 & -1.332069 & 0.000037 \\
\hline 21 & 6 & 0 & -5.263695 & 0.492283 & -0.000016 \\
\hline 22 & 1 & 0 & -5.176737 & 1.580656 & -0.000045 \\
\hline 23 & 6 & 0 & -6.655118 & -0.063474 & -0.000006 \\
\hline 24 & 1 & 0 & -6.654402 & -1.158952 & 0.000024 \\
\hline 25 & 1 & 0 & -7.217633 & 0.278389 & 0.880266 \\
\hline 26 & 1 & 0 & -7.217628 & 0.278341 & -0.880299 \\
\hline$H=-50$ & 5 & 5908 a.u & 3 a.u. & & \\
\hline
\end{tabular}


Table S16. Acetone anion $n=4$ parallel.

\begin{tabular}{|c|c|c|c|c|c|}
\hline \multirow{2}{*}{$\begin{array}{l}\text { Center } \\
\text { Number }\end{array}$} & \multirow{2}{*}{\multicolumn{2}{|c|}{$\begin{array}{l}\text { Atomic } \\
\text { Number }\end{array}$}} & Atomic & \multicolumn{2}{|c|}{ Coordinates (Angstroms) } \\
\hline & & & Type & $X \quad Y$ & Z \\
\hline 1 & 6 & 0 & -4.550993 & 0.009379 & 0.000017 \\
\hline 2 & 8 & 0 & -5.653824 & -0.584961 & -0.000024 \\
\hline 3 & 6 & 0 & -4.569394 & 1.547524 & 0.000007 \\
\hline 4 & 1 & 0 & -4.066111 & 1.959866 & 0.884553 \\
\hline 5 & 1 & 0 & -4.065321 & 1.959846 & -0.884102 \\
\hline 6 & 1 & 0 & -5.611950 & 1.877749 & -0.000460 \\
\hline 7 & 6 & 0 & -3.295405 & -0.679190 & -0.000017 \\
\hline 8 & 1 & 0 & -3.365344 & -1.767127 & -0.000071 \\
\hline 9 & 6 & 0 & -2.037277 & -0.081554 & 0.000011 \\
\hline 10 & 1 & 0 & -1.999624 & 1.011113 & 0.000038 \\
\hline 11 & 6 & 0 & -0.790905 & -0.724422 & -0.000004 \\
\hline 12 & 1 & 0 & -0.773190 & -1.816303 & 0.000007 \\
\hline 13 & 6 & 0 & 0.437048 & -0.040790 & 0.000019 \\
\hline 14 & 1 & 0 & 0.378974 & 1.053111 & 0.000054 \\
\hline 15 & 6 & 0 & 1.714056 & -0.591521 & 0.000038 \\
\hline 16 & 1 & 0 & 1.809901 & -1.679569 & 0.000084 \\
\hline 17 & 6 & 0 & 2.905049 & 0.176495 & -0.000015 \\
\hline 18 & 1 & 0 & 2.772532 & 1.263753 & -0.000034 \\
\hline 19 & 6 & 0 & 4.203278 & -0.288733 & -0.000012 \\
\hline 20 & 1 & 0 & 4.375319 & -1.367433 & 0.000017 \\
\hline 21 & 6 & 0 & 5.356056 & 0.564900 & -0.000022 \\
\hline 22 & 1 & 0 & 5.142455 & 1.638233 & -0.000069 \\
\hline 23 & 6 & 0 & 6.663653 & 0.188700 & 0.000022 \\
\hline 24 & 1 & 0 & 6.956248 & -0.860427 & -0.000051 \\
\hline 25 & 1 & 0 & 7.465715 & 0.922143 & -0.000048 \\
\hline $\begin{array}{l}H=-502 . \\
0 \text { imagin }\end{array}$ & & $\begin{array}{l}\text { IOn }=0.20 \\
1408 \text { a.u. }\end{array}$ & 29 a.u. & & \\
\hline
\end{tabular}


Table S17. Acetone neutral $n=4$ perpendicular.

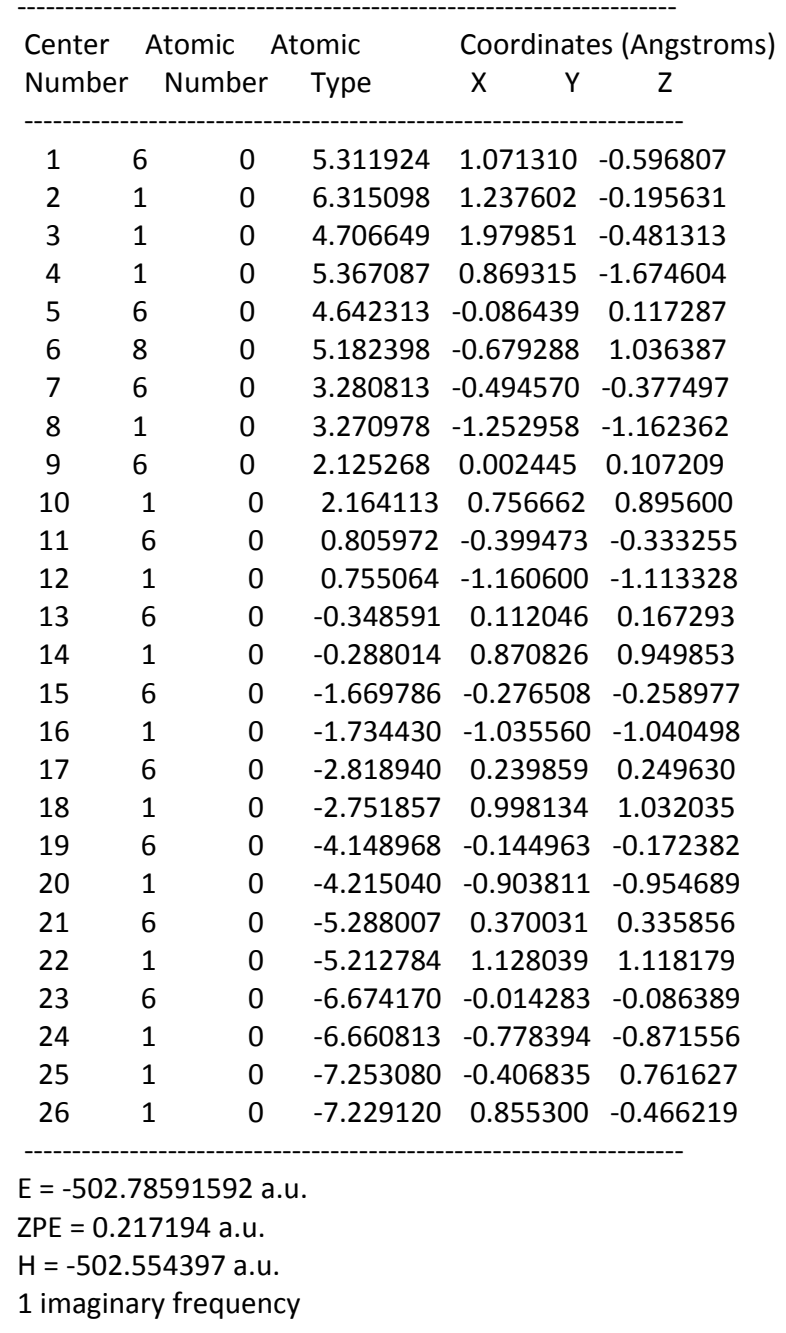


Table S18. Acetone anion $\mathrm{n}=4$ perpendicular.

\begin{tabular}{|c|c|c|c|c|c|}
\hline \multirow{2}{*}{$\begin{array}{l}\text { Center } \\
\text { Number }\end{array}$} & \multirow{2}{*}{\multicolumn{2}{|c|}{$\begin{array}{l}\text { Atomic } \\
\text { Number }\end{array}$}} & \multirow{2}{*}{$\begin{array}{l}\text { Atomic } \\
\text { Type }\end{array}$} & \multicolumn{2}{|c|}{ Coordinates (Angstroms) } \\
\hline & & & & $\begin{array}{ll}X & Y\end{array}$ & Z \\
\hline 1 & 6 & 0 & 4.542748 & -0.095582 & -0.171783 \\
\hline 2 & 8 & 0 & 5.116255 & -0.135452 & -1.256000 \\
\hline 3 & 6 & 0 & 5.146836 & -0.832112 & 1.022570 \\
\hline 4 & 1 & 0 & 5.281454 & -0.135260 & 1.860176 \\
\hline 5 & 1 & 0 & 4.440526 & -1.596890 & 1.371490 \\
\hline 6 & 1 & 0 & 6.103541 & -1.297409 & 0.759814 \\
\hline 7 & 6 & 0 & 3.278235 & 0.656687 & 0.078655 \\
\hline 8 & 1 & 0 & 3.401358 & 1.691344 & 0.405189 \\
\hline 9 & 6 & 0 & 2.029514 & 0.114915 & -0.080936 \\
\hline 10 & 1 & 0 & 1.984971 & -0.928177 & -0.414092 \\
\hline 11 & 6 & 0 & 0.766284 & 0.734008 & 0.129962 \\
\hline 12 & 1 & 0 & 0.757730 & 1.779151 & 0.447968 \\
\hline 13 & 6 & 0 & -0.451311 & 0.079976 & -0.045252 \\
\hline 14 & 1 & 0 & -0.393436 & -0.966105 & -0.368268 \\
\hline 15 & 6 & 0 & -1.744011 & 0.590699 & 0.139904 \\
\hline 16 & 1 & 0 & -1.846673 & 1.631249 & 0.457447 \\
\hline 17 & 6 & 0 & -2.920628 & -0.159592 & -0.059220 \\
\hline 18 & 1 & 0 & -2.773566 & -1.198326 & -0.377542 \\
\hline 19 & 6 & 0 & -4.235501 & 0.251528 & 0.096679 \\
\hline 20 & 1 & 0 & -4.429206 & 1.280088 & 0.411011 \\
\hline 21 & 6 & 0 & -5.364849 & -0.594825 & -0.131076 \\
\hline 22 & 1 & 0 & -5.122549 & -1.615757 & -0.445428 \\
\hline 23 & 6 & 0 & -6.689147 & -0.286423 & -0.005419 \\
\hline 24 & 1 & 0 & -7.018635 & 0.705015 & 0.303488 \\
\hline 25 & 1 & 0 & -7.464568 & -1.020982 & -0.207768 \\
\hline $\begin{array}{l}H=-50 \\
1 \mathrm{imag}\end{array}$ & .00 & 1278 a.u & 209 a.u. & & \\
\hline
\end{tabular}


2. Cartesian coordinates, absolute energies, zero-point energy corrections, absolute enthalpies, and number of imaginary frequencies for acetamide (2), acetamide enolate anion, and their $n=1-4$ vinylogues

Table S19. Acetamide neutral $n=0$.

\begin{tabular}{|c|c|c|c|c|c|}
\hline \multirow{2}{*}{$\begin{array}{l}\text { Center } \\
\text { Number }\end{array}$} & \multirow{2}{*}{\multicolumn{2}{|c|}{$\begin{array}{l}\text { Atomic } \\
\text { Number }\end{array}$}} & \multirow{2}{*}{$\begin{array}{l}\text { Atomic } \\
\text { Type }\end{array}$} & \multicolumn{2}{|c|}{ Coordinates (Angstrom } \\
\hline & & & & $\begin{array}{ll}X & Y\end{array}$ & Z \\
\hline 1 & 6 & 0 & -0.074027 & 0.143940 & -0.000125 \\
\hline 2 & 8 & 0 & -0.355973 & 1.336378 & 0.000005 \\
\hline 3 & 6 & 0 & 1.365201 & -0.346773 & 0.000000 \\
\hline 4 & 1 & 0 & 1.873459 & 0.057387 & -0.881178 \\
\hline 5 & 1 & 0 & 1.871947 & 0.053472 & 0.883871 \\
\hline 6 & 1 & 0 & 1.460519 & -1.437420 & -0.002217 \\
\hline 7 & 7 & 0 & -1.038738 & -0.827293 & 0.000045 \\
\hline 8 & 1 & 0 & -0.824333 & -1.813086 & -0.000305 \\
\hline 9 & 1 & 0 & -2.009687 & -0.543334 & 0.000216 \\
\hline
\end{tabular}

$E=-209.2269541$ a.u.

ZPE correction $=0.073539$ a.u.

$\mathrm{H}=-209.147193$ a.u.

0 imaginary frequencies

Table S20. Acetamide anion $\mathrm{n}=0$.

\begin{tabular}{|c|c|c|c|c|c|}
\hline \multirow{2}{*}{$\begin{array}{l}\text { Center } \\
\text { Number }\end{array}$} & \multirow{2}{*}{\multicolumn{2}{|c|}{$\begin{array}{l}\text { Atomic } \\
\text { Number }\end{array}$}} & Atomic & \multicolumn{2}{|c|}{ Coordinates (Angstroms) } \\
\hline & & & Type & $X \quad Y$ & Z \\
\hline 1 & 6 & 0 & 0.070531 & 0.127945 & -0.012914 \\
\hline 2 & 8 & 0 & -0.258283 & 1.353155 & 0.008192 \\
\hline 3 & 6 & 0 & 1.343305 & -0.437264 & 0.023320 \\
\hline 4 & 1 & 0 & 2.217255 & 0.207609 & 0.021869 \\
\hline 5 & 1 & 0 & 1.495058 & -1.510872 & -0.078484 \\
\hline 6 & 7 & 0 & -1.056210 & -0.805835 & -0.108451 \\
\hline 7 & 1 & 0 & -0.867688 & -1.675833 & 0.386113 \\
\hline 8 & 1 & 0 & -1.867902 & -0.349383 & 0.301686 \\
\hline
\end{tabular}

$E=-208.6150753$ a.u.

ZPE correction $=0.059732 \mathrm{a} . \mathrm{u}$.

$\mathrm{H}=-208.549872$ a.u.

0 imaginary frequencies 
Table S21. Acetamide neutral $n=1$ parallel.

\begin{tabular}{|c|c|c|c|c|c|}
\hline \multirow{2}{*}{$\begin{array}{l}\text { Center } \\
\text { Number }\end{array}$} & \multirow{2}{*}{\multicolumn{2}{|c|}{$\begin{array}{l}\text { Atomic } \\
\text { Number }\end{array}$}} & \multirow{2}{*}{$\begin{array}{l}\text { Atomic } \\
\text { Type }\end{array}$} & \multicolumn{2}{|c|}{ Coordinates (Angstroms) } \\
\hline & & & & $X \quad Y$ & Z \\
\hline 1 & 6 & 0 & -1.166793 & -0.154610 & -0.020651 \\
\hline 2 & 8 & 0 & -2.013847 & -1.033979 & 0.117489 \\
\hline 3 & 7 & 0 & -1.506507 & 1.180927 & -0.013601 \\
\hline 4 & 1 & 0 & -0.900625 & 1.865930 & -0.443043 \\
\hline 5 & 1 & 0 & -2.498310 & 1.382878 & -0.031040 \\
\hline 6 & 6 & 0 & 0.277091 & -0.496993 & -0.158536 \\
\hline 7 & 1 & 0 & 0.443842 & -1.533665 & -0.443365 \\
\hline 8 & 6 & 0 & 1.311971 & 0.313826 & 0.107070 \\
\hline 9 & 1 & 0 & 1.121753 & 1.333312 & 0.446764 \\
\hline 10 & 6 & 0 & 2.755991 & -0.081966 & 0.013324 \\
\hline 11 & 1 & 0 & 3.297206 & 0.573778 & -0.682347 \\
\hline 12 & 1 & 0 & 3.251618 & 0.017538 & 0.988573 \\
\hline 13 & 1 & 0 & 2.871285 & -1.115972 & -0.327488 \\
\hline $\begin{array}{l}E=-286 . \\
Z P E \text { corr } \\
H=-286 . \\
0 \text { imagin }\end{array}$ & $\begin{array}{l}.62 \\
\text { rect } \\
.51\end{array}$ & $\begin{array}{l}71423 \text { a.u } \\
\text { ion }=0.10 \\
1673 \text { a.u. }\end{array}$ & 7515 a.u. & & \\
\hline
\end{tabular}

Table S22. Acetamide anion $n=1$ parallel.

\begin{tabular}{|c|c|c|c|c|c|}
\hline \multirow{2}{*}{$\begin{array}{l}\text { Center } \\
\text { Number }\end{array}$} & \multirow{2}{*}{\multicolumn{2}{|c|}{$\begin{array}{l}\text { Atomic } \\
\text { Number }\end{array}$}} & \multirow{2}{*}{$\begin{array}{l}\text { Atomic } \\
\text { Type }\end{array}$} & \multicolumn{2}{|c|}{ Coordinates (Angstroms) } \\
\hline & & & & $X \quad Y$ & Z \\
\hline 1 & 6 & 0 & 1.058582 & -0.175385 & -0.008267 \\
\hline 2 & 8 & 0 & 2.064821 & -0.935693 & -0.016756 \\
\hline 3 & 7 & 0 & 1.345355 & 1.238635 & -0.067321 \\
\hline 4 & 1 & 0 & 0.705782 & 1.812946 & 0.474456 \\
\hline 5 & 1 & 0 & 2.303968 & 1.381377 & 0.236364 \\
\hline 6 & 6 & 0 & -0.293583 & -0.589861 & 0.023201 \\
\hline 7 & 1 & 0 & -0.438950 & -1.667588 & 0.084828 \\
\hline 8 & 6 & 0 & -1.435587 & 0.251512 & -0.043640 \\
\hline 9 & 1 & 0 & -1.236598 & 1.320223 & -0.180181 \\
\hline 10 & 6 & 0 & -2.767712 & -0.084046 & 0.016350 \\
\hline 11 & 1 & 0 & -3.095125 & -1.116866 & 0.136705 \\
\hline 12 & 1 & 0 & -3.545329 & 0.671684 & -0.072741 \\
\hline $\begin{array}{l}E=-286 . \\
Z P E \text { corr } \\
H=-285 \\
0 \text { imagin }\end{array}$ & & $\begin{array}{l}12881 \text { a.u } \\
\text { ion }=0.09 \\
035 \text { a.u. } \\
\text { y frequenc }\end{array}$ & 64 a.u. & & \\
\hline
\end{tabular}


Table S23. Acetamide neutral $n=1$ perpendicular.

\begin{tabular}{|c|c|c|c|c|c|}
\hline \multirow{2}{*}{$\begin{array}{l}\text { Center } \\
\text { Number }\end{array}$} & \multirow{2}{*}{\multicolumn{2}{|c|}{$\begin{array}{l}\text { Atomic } \\
\text { Number }\end{array}$}} & \multirow{2}{*}{$\begin{array}{l}\text { Atomic } \\
\text { Type }\end{array}$} & \multicolumn{2}{|c|}{ Coordinates (Angstroms) } \\
\hline & & & & $X \quad Y$ & Z \\
\hline 1 & 6 & 0 & -1.148738 & -0.143084 & 0.076719 \\
\hline 2 & 8 & 0 & -1.706246 & -1.216956 & -0.121535 \\
\hline 3 & 7 & 0 & -1.799953 & 1.053211 & -0.046980 \\
\hline 4 & 1 & 0 & -1.324428 & 1.934122 & 0.083153 \\
\hline 5 & 1 & 0 & -2.775871 & 1.055506 & -0.316045 \\
\hline 6 & 6 & 0 & 0.295506 & -0.045631 & 0.493211 \\
\hline 7 & 1 & 0 & 0.483399 & -0.144901 & 1.563059 \\
\hline 8 & 6 & 0 & 1.309517 & 0.040870 & -0.374125 \\
\hline 9 & 1 & 0 & 1.084705 & 0.105396 & -1.440458 \\
\hline 10 & 6 & 0 & 2.764953 & 0.034564 & -0.002855 \\
\hline 11 & 1 & 0 & 3.270503 & 0.939993 & -0.364814 \\
\hline 12 & 1 & 0 & 2.904675 & -0.027308 & 1.081880 \\
\hline 13 & 1 & 0 & 3.279226 & -0.819942 & -0.463326 \\
\hline $\begin{array}{l}E=-286 . \\
Z P E \text { corre } \\
H=-286 . \\
1 \text { imagin }\end{array}$ & $\begin{array}{l}621 \\
\text { ecti } \\
.50 \\
\text { lary }\end{array}$ & $\begin{array}{l}12903 \text { a.u } \\
\text { tion }=0.10 \\
7148 \text { a.u. } \\
\text { y frequen }\end{array}$ & 5678 a.u. & & \\
\hline
\end{tabular}

Table S24. Acetamide anion $n=1$ perpendicular.

\begin{tabular}{|c|c|c|c|c|c|}
\hline \multirow{2}{*}{$\begin{array}{l}\text { Center } \\
\text { Number }\end{array}$} & \multirow{2}{*}{\multicolumn{2}{|c|}{$\begin{array}{l}\text { Atomic } \\
\text { Number }\end{array}$}} & \multirow{2}{*}{$\begin{array}{l}\text { Atomic } \\
\text { Type }\end{array}$} & \multicolumn{2}{|c|}{ Coordinates (Angstroms) } \\
\hline & & & & $X \quad Y$ & Z \\
\hline 1 & 6 & 0 & -1.063300 & -0.154142 & 0.099275 \\
\hline 2 & 8 & 0 & -1.735974 & -1.161979 & -0.163817 \\
\hline 3 & 7 & 0 & -1.689412 & 1.089649 & -0.035422 \\
\hline 4 & 1 & 0 & -1.090793 & 1.902898 & -0.057173 \\
\hline 5 & 1 & 0 & -2.569205 & 1.127390 & -0.544108 \\
\hline 6 & 6 & 0 & 0.339810 & -0.093657 & 0.578738 \\
\hline 7 & 1 & 0 & 0.480742 & -0.105431 & 1.660367 \\
\hline 8 & 6 & 0 & 1.428119 & -0.025115 & -0.301564 \\
\hline 9 & 1 & 0 & 1.147872 & -0.037129 & -1.364327 \\
\hline 10 & 6 & 0 & 2.799420 & 0.050545 & -0.071701 \\
\hline 11 & 1 & 0 & 3.217132 & 0.049383 & 0.935739 \\
\hline 12 & 1 & 0 & 3.503637 & 0.065397 & -0.900504 \\
\hline $\begin{array}{l}E=-286 . \\
Z P E \text { corre } \\
H=-285 . \\
1 \text { imagina }\end{array}$ & $\begin{array}{l}.00 \\
\text { rect } \\
5.90\end{array}$ & $\begin{array}{l}52684 \text { a.u } \\
\text { tion }=0.09 \\
69991 \text { a.u. } \\
\text { y frequenc }\end{array}$ & 748 a.u. & & \\
\hline
\end{tabular}


Table S25. Acetamide neutral $n=2$ parallel.

\begin{tabular}{|c|c|c|c|c|c|}
\hline \multirow{2}{*}{$\begin{array}{l}\text { Center } \\
\text { Number }\end{array}$} & \multirow{2}{*}{\multicolumn{2}{|c|}{$\begin{array}{l}\text { Atomic } \\
\text { Number }\end{array}$}} & \multirow{2}{*}{$\begin{array}{l}\text { Atomic } \\
r \quad \text { Type }\end{array}$} & \multicolumn{2}{|c|}{ Coordinates (Angstroms) } \\
\hline & & & & $X \quad Y$ & Z \\
\hline 1 & 6 & 0 & -2.301293 & -0.127592 & -0.002420 \\
\hline 2 & 8 & 0 & -3.216147 & -0.926976 & 0.188504 \\
\hline 3 & 7 & 0 & -2.533523 & 1.232283 & -0.050598 \\
\hline 4 & 1 & 0 & -1.890933 & 1.840837 & -0.538456 \\
\hline 5 & 1 & 0 & -3.508213 & 1.505448 & -0.071870 \\
\hline 6 & 6 & 0 & -0.897788 & -0.590582 & -0.145279 \\
\hline 7 & 1 & 0 & -0.816952 & -1.654836 & -0.353879 \\
\hline 8 & 6 & 0 & 0.211431 & 0.159238 & 0.028887 \\
\hline 9 & 1 & 0 & 0.116574 & 1.212980 & 0.295206 \\
\hline 10 & 6 & 0 & 1.567289 & -0.349548 & -0.081162 \\
\hline 11 & 1 & 0 & 1.677823 & -1.403997 & -0.338109 \\
\hline 12 & 6 & 0 & 2.667880 & 0.402852 & 0.114244 \\
\hline 13 & 1 & 0 & 2.539517 & 1.455818 & 0.373690 \\
\hline 14 & 6 & 0 & 4.080430 & -0.086160 & 0.007758 \\
\hline 15 & 1 & 0 & 4.618258 & 0.055177 & 0.955588 \\
\hline 16 & 1 & 0 & 4.123218 & -1.148735 & -0.254405 \\
\hline 17 & 1 & 0 & 4.636849 & 0.477885 & -0.753770 \\
\hline
\end{tabular}

$E=-364.0356917$ a.u.

ZPE correction $=0.141036$ a.u.

$\mathrm{H}=-363.884536$ a.u.

0 imaginary frequencies

Table S26. Acetamide anion $n=2$ parallel.

\begin{tabular}{|c|c|c|c|c|c|}
\hline \multirow{2}{*}{$\begin{array}{l}\text { Center } \\
\text { Number }\end{array}$} & \multirow{2}{*}{\multicolumn{2}{|c|}{$\begin{array}{l}\text { Atomic } \\
\text { Number }\end{array}$}} & Atomic & \multicolumn{2}{|c|}{ Coordinates (Angstroms } \\
\hline & & & r Type & $\begin{array}{ll}X & Y\end{array}$ & Z \\
\hline 1 & 6 & 0 & 2.201915 & -0.122801 & -0.009996 \\
\hline 2 & 8 & 0 & 3.262096 & -0.794053 & -0.038189 \\
\hline 3 & 7 & 0 & 2.347244 & 1.300336 & -0.047878 \\
\hline 4 & 1 & 0 & 1.643587 & 1.819275 & 0.467396 \\
\hline 5 & 1 & 0 & 3.286704 & 1.552667 & 0.240970 \\
\hline 6 & 6 & 0 & 0.886061 & -0.673793 & 0.021531 \\
\hline 7 & 1 & 0 & 0.857067 & -1.760980 & 0.068711 \\
\hline 8 & 6 & 0 & -0.322021 & 0.039693 & -0.020553 \\
\hline 9 & 1 & 0 & -0.253200 & 1.128216 & -0.125192 \\
\hline 10 & 6 & 5 & -1.620418 & -0.456451 & 0.030907 \\
\hline 11 & 1 & 1 & -1.765810 & -1.535523 & 0.124485 \\
\hline 12 & 6 & 5 & -2.783189 & 0.368641 & -0.035361 \\
\hline 13 & 1 & 1 & -2.580711 & 1.441837 & -0.131294 \\
\hline 14 & 6 & 5 & -4.099022 & -0.002315 & 0.005659 \\
\hline 15 & 1 & 1 & -4.397176 & -1.046481 & 0.096744 \\
\hline 16 & 1 & 1 & -4.897903 & 0.733215 & -0.054292 \\
\hline
\end{tabular}

$E=-363.4647018$ a.u.

ZPE correction $=0.126943$ a.u.

$\mathrm{H}=-363.328161$ a.u.

0 imaginary frequencies 
Table S27. Acetamide neutral $n=2$ perpendicular.

\begin{tabular}{|c|c|c|c|c|c|}
\hline \multirow{2}{*}{$\begin{array}{l}\text { Center } \\
\text { Number }\end{array}$} & \multirow{2}{*}{\multicolumn{2}{|c|}{$\begin{array}{l}\text { Atomic } \\
\text { Number }\end{array}$}} & \multirow{2}{*}{$\begin{array}{l}\text { Atomic } \\
\text { Type }\end{array}$} & \multicolumn{2}{|c|}{ Coordinates (Angstroms) } \\
\hline & & & & $X \quad Y$ & Z \\
\hline 1 & 6 & 0 & -2.283243 & -0.137187 & 0.042238 \\
\hline 2 & 8 & 0 & -2.837896 & -1.182897 & -0.278356 \\
\hline 3 & 7 & 0 & -2.896766 & 1.080077 & -0.066734 \\
\hline 4 & 1 & 0 & -2.423839 & 1.937854 & 0.177598 \\
\hline 5 & 1 & 0 & -3.844506 & 1.123220 & -0.419973 \\
\hline 6 & 6 & 0 & -0.884697 & -0.102000 & 0.595545 \\
\hline 7 & 1 & 0 & -0.796885 & -0.282980 & 1.667062 \\
\hline 8 & 6 & 0 & 0.213100 & 0.017350 & -0.172585 \\
\hline 9 & 1 & 0 & 0.093933 & 0.162188 & -1.247705 \\
\hline 10 & 6 & 0 & 1.579566 & -0.043348 & 0.326393 \\
\hline 11 & 1 & 0 & 1.703619 & -0.201165 & 1.399350 \\
\hline 12 & 6 & 0 & 2.670488 & 0.077163 & -0.452709 \\
\hline 13 & 1 & 0 & 2.530385 & 0.230392 & -1.524751 \\
\hline 14 & 6 & 0 & 4.089356 & 0.012235 & 0.028402 \\
\hline 15 & 1 & 0 & 4.634750 & -0.807447 & -0.459842 \\
\hline 16 & 1 & 0 & 4.632569 & 0.936534 & -0.213331 \\
\hline 17 & 1 & 0 & 4.143084 & -0.141226 & 1.111874 \\
\hline
\end{tabular}

$E=-364.0289457$ a.u.

ZPE correction $=0.140040 \mathrm{a} . \mathrm{u}$.

$\mathrm{H}=-363.879225$ a.u.

1 imaginary frequency

Table S28. Acetamide anion $n=2$ perpendicular.

\begin{tabular}{|c|c|c|c|c|c|}
\hline \multirow{2}{*}{$\begin{array}{l}\text { Center } \\
\text { Number }\end{array}$} & \multirow{2}{*}{\multicolumn{2}{|c|}{$\begin{array}{l}\text { Atomic } \\
\text { Numbe }\end{array}$}} & \multirow{2}{*}{$\begin{array}{l}\text { Atomic } \\
\text { Type }\end{array}$} & \multicolumn{2}{|c|}{ Coordinates (Angstron } \\
\hline & & & & $X \quad Y$ & Z \\
\hline 1 & 6 & 0 & -2.198540 & -0.139816 & 0.029686 \\
\hline 2 & 8 & 0 & -2.841113 & -1.112269 & -0.383344 \\
\hline 3 & 7 & 0 & -2.767298 & 1.126130 & -0.084554 \\
\hline 4 & 1 & 0 & -2.162984 & 1.926469 & 0.035348 \\
\hline 5 & 1 & 0 & -3.586075 & 1.227088 & -0.676454 \\
\hline 6 & 6 & 0 & -0.864492 & -0.160965 & 0.690305 \\
\hline 7 & 1 & 0 & -0.869558 & -0.252121 & 1.776888 \\
\hline 8 & 6 & 0 & 0.321628 & -0.071064 & -0.019936 \\
\hline 9 & 1 & 0 & 0.215056 & 0.006029 & -1.109959 \\
\hline 10 & 6 & 0 & 1.651874 & -0.066867 & 0.440468 \\
\hline 11 & 1 & 1 & 1.831116 & -0.145640 & 1.516484 \\
\hline 12 & 6 & 5 & 2.773342 & 0.038933 & -0.420615 \\
\hline 13 & 1 & 1 & 2.521753 & 0.110456 & -1.486851 \\
\hline 14 & 6 & 5 & 4.116884 & 0.065755 & -0.120194 \\
\hline 15 & 1 & 1 & 4.477631 & -0.002596 & 0.906352 \\
\hline 16 & 1 & 0 & 4.868872 & 0.149691 & -0.901457 \\
\hline
\end{tabular}

$E=-363.4355351$ a.u.

ZPE correction $=0.124726$ a.u.

$\mathrm{H}=-363.30139$ a.u.

1 imaginary frequency 
Table S29. Acetamide neutral $n=3$ parallel.

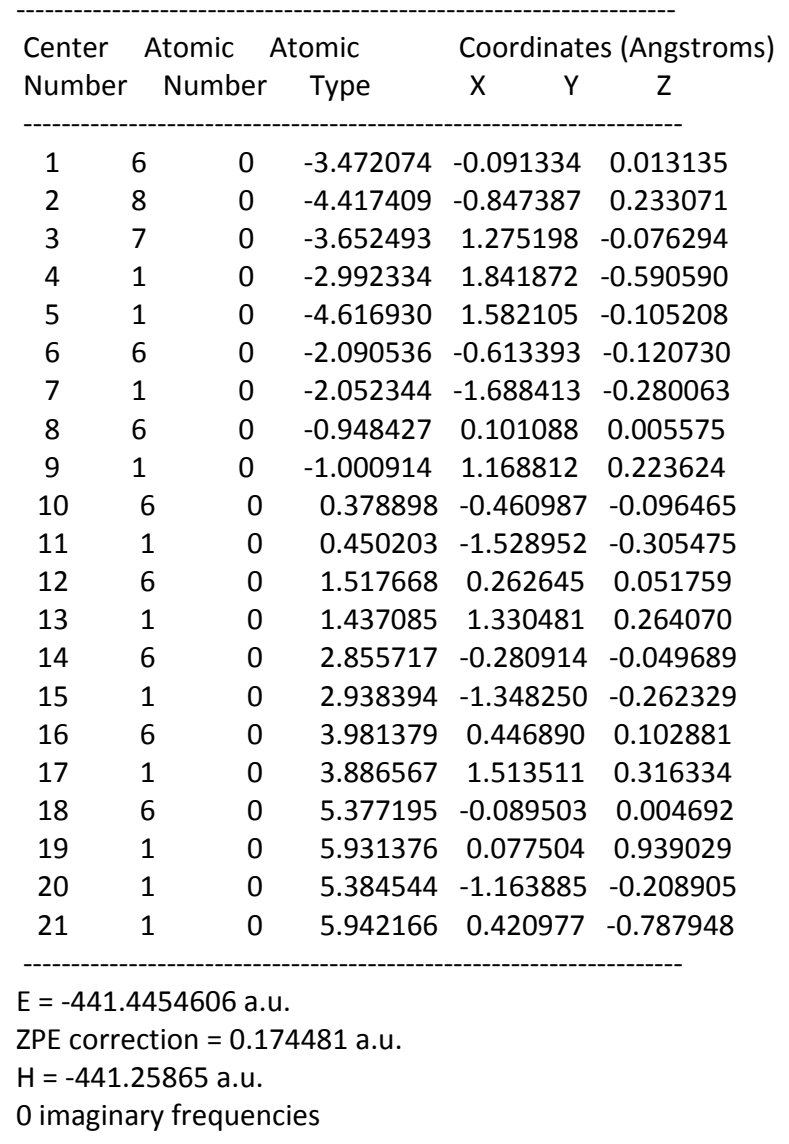


Table S30. Acetamide anion $\mathrm{n}=3$ parallel.

\begin{tabular}{|c|c|c|c|c|c|}
\hline \multirow{2}{*}{$\begin{array}{l}\text { Center } \\
\text { Number }\end{array}$} & \multicolumn{2}{|r|}{ Atomic } & Atomic & \multicolumn{2}{|c|}{ Coordinates (Angstroms } \\
\hline & & Number & r Type & $X \quad Y$ & Z \\
\hline 1 & 6 & 0 & 3.373552 & -0.045603 & -0.012402 \\
\hline 2 & 8 & 0 & 4.461029 & -0.662610 & -0.053525 \\
\hline 3 & 7 & 0 & 3.433595 & 1.375800 & -0.031615 \\
\hline 4 & 1 & 0 & 2.696239 & 1.856510 & 0.471941 \\
\hline 5 & 1 & 0 & 4.357331 & 1.693374 & 0.241097 \\
\hline 6 & 6 & 0 & 2.085730 & -0.677016 & 0.015539 \\
\hline 7 & 1 & 0 & 2.125008 & -1.763826 & 0.053342 \\
\hline 8 & 6 & 0 & 0.845806 & -0.039689 & -0.016416 \\
\hline 9 & 1 & 0 & 0.841632 & 1.051623 & -0.104396 \\
\hline 10 & 6 & 0 & -0.423482 & -0.627874 & 0.030843 \\
\hline 11 & 1 & 0 & -0.487662 & -1.715418 & 0.108934 \\
\hline 12 & 6 & 0 & -1.627515 & 0.107686 & -0.018311 \\
\hline 13 & 1 & 0 & -1.516497 & 1.195456 & -0.098105 \\
\hline 14 & 6 & 0 & -2.924910 & -0.372720 & 0.022724 \\
\hline 15 & 1 & 0 & -3.083472 & -1.450926 & 0.100698 \\
\hline 16 & 6 & 0 & -4.083722 & 0.465350 & -0.031938 \\
\hline 17 & 1 & 0 & -3.876895 & 1.537899 & -0.111428 \\
\hline 18 & 6 & 0 & -5.394595 & 0.089827 & 0.002465 \\
\hline 19 & 1 & 0 & -5.687921 & -0.956460 & 0.080023 \\
\hline 20 & 1 & 0 & -6.196343 & 0.822276 & -0.047622 \\
\hline
\end{tabular}

$E=-440.8846964$ a.u.

ZPE correction $=0.160364$ a.u.

$\mathrm{H}=-440.712556$ a.u.

0 imaginary frequencies 
Table S31. Acetamide neutral $n=3$ perpendicular.

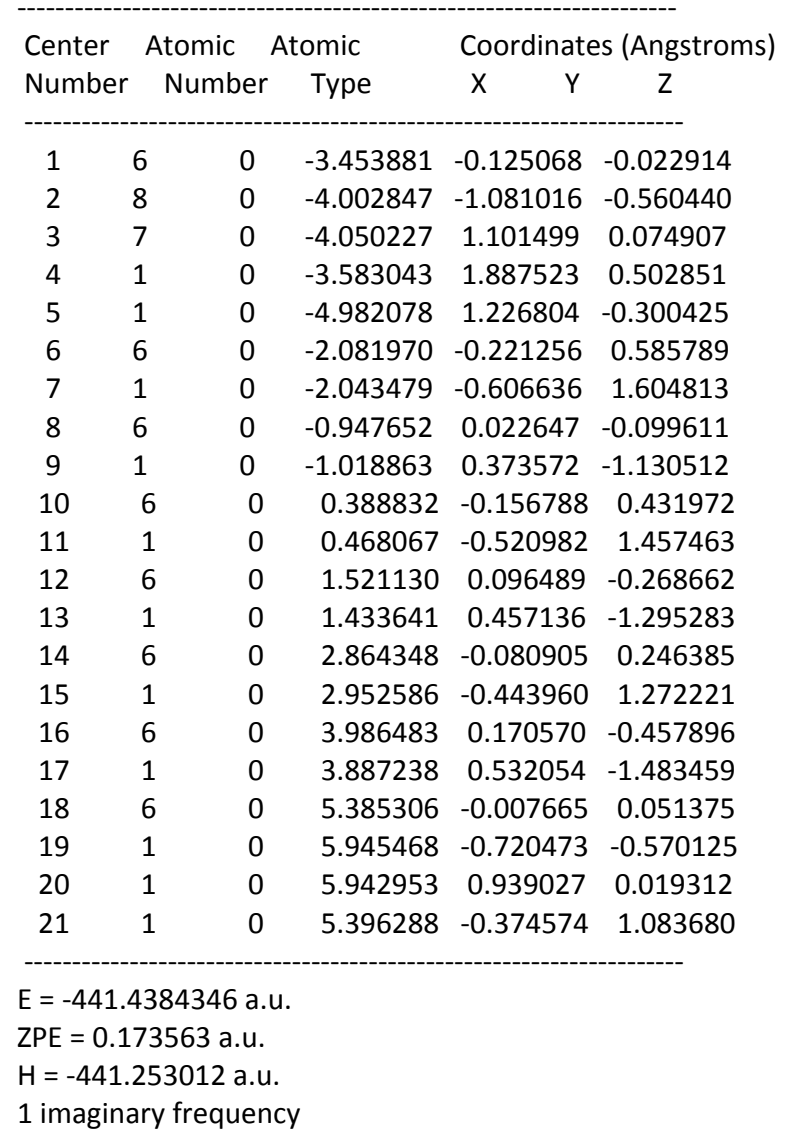


Table S32. Acetamide anion $\mathrm{n}=3$ perpendicular.

\begin{tabular}{|c|c|c|c|c|c|}
\hline \multirow{2}{*}{$\begin{array}{l}\text { Center } \\
\text { Number }\end{array}$} & \multicolumn{2}{|r|}{ Atomic } & Atomic & \multicolumn{2}{|c|}{ Coordinates (Angstroms } \\
\hline & & Number & r Type & $\begin{array}{ll}X & Y\end{array}$ & Z \\
\hline 1 & 6 & 0 & -3.363258 & 0.001115 & 0.134141 \\
\hline 2 & 8 & 0 & -3.977360 & 0.043469 & 1.204318 \\
\hline 3 & 7 & 0 & -3.904936 & -0.712088 & -0.925971 \\
\hline 4 & 1 & 0 & -3.311603 & -0.926902 & -1.714525 \\
\hline 5 & 1 & 0 & -4.694021 & -1.320882 & -0.736544 \\
\hline 6 & 6 & 0 & -2.075491 & 0.687243 & -0.175445 \\
\hline 7 & 1 & 0 & -2.157404 & 1.692598 & -0.589293 \\
\hline 8 & 6 & 0 & -0.848115 & 0.102436 & 0.041452 \\
\hline 9 & 1 & 0 & -0.862707 & -0.907924 & 0.467039 \\
\hline 10 & 6 & 0 & 0.444041 & 0.625553 & -0.207908 \\
\hline 11 & 1 & 1 & 0.518778 & 1.626345 & -0.640412 \\
\hline 12 & 6 & 5 & 1.627654 & -0.074702 & 0.066862 \\
\hline 13 & 1 & 1 & 1.495601 & -1.072470 & 0.503871 \\
\hline 14 & 6 & 5 & 2.946298 & 0.322965 & -0.140097 \\
\hline 15 & 1 & 0 & 3.133454 & 1.308342 & -0.574768 \\
\hline 16 & 6 & 5 & 4.075621 & -0.484395 & 0.184794 \\
\hline 17 & 1 & 1 & 3.832349 & -1.461394 & 0.618317 \\
\hline 18 & 6 & 5 & 5.406229 & -0.202480 & 0.026618 \\
\hline 19 & 1 & 1 & 5.745491 & 0.743893 & -0.393971 \\
\hline 20 & 1 & 0 & 6.175621 & -0.911156 & 0.325038 \\
\hline
\end{tabular}

$E=-440.8597631$ a.u.

ZPE correction $=0.158434$ a.u.

$\mathrm{H}=-440.689811$ a.u.

1 imaginary frequency 
Table S33. Acetamide neutral $n=4$ parallel.

\begin{tabular}{|c|c|c|c|c|c|}
\hline \multirow{2}{*}{$\begin{array}{l}\text { Center } \\
\text { Number }\end{array}$} & \multirow{2}{*}{\multicolumn{2}{|c|}{$\begin{array}{l}\text { Atomic } \\
\text { Number }\end{array}$}} & \multirow{2}{*}{$\begin{array}{l}\text { Atomic } \\
\text { Type }\end{array}$} & \multicolumn{2}{|c|}{ Coordinates (Angstroms) } \\
\hline & & & & $X \quad Y$ & Z \\
\hline 1 & 6 & 0 & -4.662952 & -0.053433 & 0.025761 \\
\hline 2 & 8 & 0 & -5.625694 & -0.781809 & 0.264282 \\
\hline 3 & 7 & 0 & -4.812316 & 1.315003 & -0.093220 \\
\hline 4 & 1 & 0 & -4.141552 & 1.854805 & -0.622539 \\
\hline 5 & 1 & 0 & -5.769905 & 1.642037 & -0.128444 \\
\hline 6 & 6 & 0 & -3.295355 & -0.610758 & -0.098848 \\
\hline 7 & 1 & 0 & -3.282956 & -1.690901 & -0.223743 \\
\hline 8 & 6 & 0 & -2.134604 & 0.080448 & -0.003252 \\
\hline 9 & 1 & 0 & -2.160411 & 1.155667 & 0.180057 \\
\hline 10 & 6 & 0 & -0.824091 & -0.515265 & -0.095435 \\
\hline 11 & 1 & 0 & -0.778449 & -1.590905 & -0.268955 \\
\hline 12 & 6 & 0 & 0.335637 & 0.186953 & 0.021766 \\
\hline 13 & 1 & 0 & 0.279342 & 1.262810 & 0.198675 \\
\hline 14 & 6 & 0 & 1.652398 & -0.387536 & -0.068556 \\
\hline 15 & 1 & 0 & 1.713683 & -1.462366 & -0.245885 \\
\hline 16 & 6 & 0 & 2.803913 & 0.324711 & 0.054869 \\
\hline 17 & 1 & 0 & 2.738847 & 1.399640 & 0.233381 \\
\hline 18 & 6 & 0 & 4.131796 & -0.242114 & -0.032877 \\
\hline 19 & 1 & 0 & 4.196623 & -1.317037 & -0.211841 \\
\hline 20 & 6 & 0 & 5.271403 & 0.470358 & 0.092110 \\
\hline 21 & 1 & 0 & 5.196378 & 1.544757 & 0.271524 \\
\hline 22 & 6 & 0 & 6.656866 & -0.094272 & 0.005214 \\
\hline 23 & 1 & 0 & 7.218455 & 0.090105 & 0.931956 \\
\hline 24 & 1 & 0 & 6.643429 & -1.174662 & -0.175740 \\
\hline 25 & 1 & 0 & 7.228213 & 0.380961 & -0.804675 \\
\hline $\begin{array}{l}\text { ZPE corre } \\
\mathrm{H}=-518 .\end{array}$ & 3.63 & $\begin{array}{l}33279 \text { a.u } \\
\text { y frequen }\end{array}$ & 68 a.u. & & \\
\hline
\end{tabular}


Table S34. Acetamide anion $n=4$ parallel.

\begin{tabular}{|c|c|c|c|c|c|}
\hline \multirow{2}{*}{$\begin{array}{l}\text { Center } \\
\text { Number }\end{array}$} & \multirow{2}{*}{\multicolumn{2}{|c|}{$\begin{array}{c}\text { Atomic } \\
\text { Number }\end{array}$}} & \multirow{2}{*}{$\begin{array}{l}\text { Atomic } \\
\text { Type }\end{array}$} & \multicolumn{2}{|c|}{ Coordinates (Angstroms) } \\
\hline & & & & $\begin{array}{ll}X & Y\end{array}$ & Z \\
\hline 1 & 6 & 0 & 4.559756 & 0.047226 & -0.015955 \\
\hline 2 & 8 & 0 & 5.665605 & -0.528701 & -0.072919 \\
\hline 3 & 7 & 0 & 4.557251 & 1.464129 & -0.015151 \\
\hline 4 & 1 & 0 & 3.799419 & 1.913279 & 0.485993 \\
\hline 5 & 1 & 0 & 5.468114 & 1.828437 & 0.240418 \\
\hline 6 & 6 & 0 & 3.295349 & -0.642389 & 0.010571 \\
\hline 7 & 1 & 0 & 3.384188 & -1.726258 & 0.041131 \\
\hline 8 & 6 & 0 & 2.034673 & -0.061030 & -0.014241 \\
\hline 9 & 1 & 0 & 1.978059 & 1.029029 & -0.093175 \\
\hline 10 & 6 & 0 & 0.790663 & -0.713140 & 0.030199 \\
\hline 11 & 1 & 0 & 0.782054 & -1.802873 & 0.098977 \\
\hline 12 & 6 & 0 & -0.439770 & -0.039096 & -0.010185 \\
\hline 13 & 1 & 0 & -0.387531 & 1.053116 & -0.080077 \\
\hline 14 & 6 & 0 & -1.716580 & -0.592779 & 0.028889 \\
\hline 15 & 1 & 0 & -1.809414 & -1.678990 & 0.097064 \\
\hline 16 & 6 & 0 & -2.908024 & 0.171319 & -0.015273 \\
\hline 17 & 1 & 0 & -2.776393 & 1.256627 & -0.085143 \\
\hline 18 & 6 & 0 & -4.207513 & -0.291946 & 0.019318 \\
\hline 19 & 1 & 0 & -4.380453 & -1.368306 & 0.089100 \\
\hline 20 & 6 & 0 & -5.358692 & 0.561203 & -0.032228 \\
\hline 21 & 1 & 0 & -5.142926 & 1.631942 & -0.102672 \\
\hline 22 & 6 & 0 & -6.668029 & 0.189972 & -0.003938 \\
\hline 23 & 1 & 0 & -6.964000 & -0.855985 & 0.064607 \\
\hline 24 & 1 & 0 & -7.467712 & 0.924654 & -0.049754 \\
\hline $\begin{array}{l}\text { ZPE corre } \\
\mathrm{H}=-518 . \\
0 \text { imagin }\end{array}$ & & $\begin{array}{l}0 n=0.19 \\
4822 \text { a.u. } \\
\text { frequenc }\end{array}$ & 48 a.u. & & \\
\hline
\end{tabular}


Table S35. Acetamide neutral $n=4$ perpendicular.

\begin{tabular}{|c|c|c|c|c|c|}
\hline \multirow{2}{*}{$\begin{array}{l}\text { Center } \\
\text { Number }\end{array}$} & \multirow{2}{*}{\multicolumn{2}{|c|}{$\begin{array}{l}\text { Atomic } \\
\text { Number }\end{array}$}} & \multirow{2}{*}{$\begin{array}{l}\text { Atomic } \\
\text { Type }\end{array}$} & \multicolumn{2}{|c|}{ Coordinates (Angstroms) } \\
\hline & & & & $X \quad Y$ & Z \\
\hline 1 & 6 & 0 & 4.644715 & -0.043265 & 0.112958 \\
\hline 2 & 8 & 0 & 5.192586 & -0.439535 & 1.136310 \\
\hline 3 & 7 & 0 & 5.227767 & 0.866531 & -0.724811 \\
\hline 4 & 1 & 0 & 4.753052 & 1.213552 & -1.545549 \\
\hline 5 & 1 & 0 & 6.141591 & 1.234813 & -0.491664 \\
\hline 6 & 6 & 0 & 3.290194 & -0.535335 & -0.316712 \\
\hline 7 & 1 & 0 & 3.281395 & -1.474200 & -0.870458 \\
\hline 8 & 6 & 0 & 2.135520 & 0.054255 & 0.054603 \\
\hline 9 & 1 & 0 & 2.178151 & 0.974364 & 0.640031 \\
\hline 10 & 6 & 0 & 0.816398 & -0.450782 & -0.261175 \\
\hline 11 & 1 & 0 & 0.764536 & -1.379916 & -0.830740 \\
\hline 12 & 6 & 0 & -0.337865 & 0.161417 & 0.111257 \\
\hline 13 & 1 & 0 & -0.275957 & 1.088379 & 0.684573 \\
\hline 14 & 6 & 0 & -1.659623 & -0.326638 & -0.193283 \\
\hline 15 & 1 & 0 & -1.725562 & -1.257363 & -0.759406 \\
\hline 16 & 6 & 0 & -2.808129 & 0.296283 & 0.179535 \\
\hline 17 & 1 & 0 & -2.739659 & 1.226210 & 0.747131 \\
\hline 18 & 6 & 0 & -4.138901 & -0.186858 & -0.121913 \\
\hline 19 & 1 & 0 & -4.206323 & -1.114852 & -0.693265 \\
\hline 20 & 6 & 0 & -5.277267 & 0.431947 & 0.255234 \\
\hline 21 & 1 & 0 & -5.200874 & 1.359176 & 0.826639 \\
\hline 22 & 6 & 0 & -6.663975 & -0.051528 & -0.046738 \\
\hline 23 & 1 & 0 & -7.233956 & -0.224682 & 0.877055 \\
\hline 24 & 1 & 0 & -7.227253 & 0.694323 & -0.625336 \\
\hline 25 & 1 & 0 & -6.650604 & -0.986212 & -0.618408 \\
\hline $\begin{array}{l}\text { ZPE corre } \\
\mathrm{H}=-518 .\end{array}$ & 62 & 27515 a. & 81 a.u. & & \\
\hline
\end{tabular}


Table S36. Acetamide anion $\mathrm{n}=4$ perpendicular.

\begin{tabular}{|c|c|c|c|c|c|}
\hline \multirow{2}{*}{$\begin{array}{l}\text { Center } \\
\text { Number }\end{array}$} & \multirow{2}{*}{\multicolumn{2}{|c|}{$\begin{array}{l}\text { Atomic } \\
\text { Number }\end{array}$}} & Atomic & \multicolumn{2}{|c|}{ Coordinates (Angstroms) } \\
\hline & & & Type & $X \quad Y$ & Z \\
\hline 1 & 6 & 0 & 4.544955 & -0.133183 & -0.125780 \\
\hline 2 & 8 & 0 & 5.151921 & -0.343362 & -1.178406 \\
\hline 3 & 7 & 0 & 5.053550 & -0.624101 & 1.065257 \\
\hline 4 & 1 & 0 & 4.460249 & -0.633117 & 1.882457 \\
\hline 5 & 1 & 0 & 5.822944 & -1.282938 & 1.020078 \\
\hline 6 & 6 & 0 & 3.289495 & 0.662764 & 0.019302 \\
\hline 7 & 1 & 0 & 3.419709 & 1.728244 & 0.209837 \\
\hline 8 & 6 & 0 & 2.039384 & 0.107496 & -0.077593 \\
\hline 9 & 1 & 0 & 1.995049 & -0.968850 & -0.277216 \\
\hline 10 & 6 & 0 & 0.777697 & 0.750764 & 0.046561 \\
\hline 11 & 1 & 0 & 0.768999 & 1.826620 & 0.236093 \\
\hline 12 & 6 & 0 & -0.440160 & 0.080257 & -0.052897 \\
\hline 13 & 1 & 0 & -0.380571 & -0.997470 & -0.244933 \\
\hline 14 & 6 & 0 & -1.733290 & 0.608251 & 0.064143 \\
\hline 15 & 1 & 0 & -1.838433 & 1.679315 & 0.253446 \\
\hline 16 & 6 & 0 & -2.908546 & -0.163013 & -0.045194 \\
\hline 17 & 1 & 0 & -2.758925 & -1.232278 & -0.235273 \\
\hline 18 & 6 & 0 & -4.224104 & 0.261201 & 0.058139 \\
\hline 19 & 1 & 0 & -4.420490 & 1.319524 & 0.246748 \\
\hline 20 & 6 & 0 & -5.351496 & -0.609137 & -0.067512 \\
\hline 21 & 1 & 0 & -5.106668 & -1.659916 & -0.256394 \\
\hline 22 & 6 & 0 & -6.676267 & -0.290286 & 0.018733 \\
\hline 23 & 1 & 0 & -7.007841 & 0.730841 & 0.203780 \\
\hline 24 & 1 & 0 & -7.450254 & -1.045056 & -0.095583 \\
\hline $\begin{array}{l}\text { ZPE corr } \\
H=-518 \\
1 \text { imagin }\end{array}$ & & y frequen & 60 a.u. & & \\
\hline
\end{tabular}


3. Cartesian coordinates, absolute energies, zero-point energy corrections, absolute enthalpies, and number of imaginary frequencies for acetic acid (3), acetic acid enolate anion, and their $n=1-4$ vinylogues

Table S37. Acetic acid neutral $n=0$.

\begin{tabular}{|c|c|c|c|c|c|}
\hline \multirow{2}{*}{$\begin{array}{l}\text { Center } \\
\text { Number }\end{array}$} & \multirow{2}{*}{\multicolumn{2}{|c|}{$\begin{array}{l}\text { Atomic } \\
\text { Number }\end{array}$}} & \multirow{2}{*}{$\begin{array}{l}\text { Atomic } \\
\text { Type }\end{array}$} & \multicolumn{2}{|c|}{ Coordinates (Angstrom } \\
\hline & & & & $\begin{array}{ll}X & Y\end{array}$ & z \\
\hline 1 & 6 & 0 & 0.088758 & 0.125663 & -0.000005 \\
\hline 2 & 8 & 0 & 0.634851 & 1.208264 & 0.000000 \\
\hline 3 & 8 & 0 & 0.790122 & -1.040080 & -0.000004 \\
\hline 4 & 1 & 0 & 1.737241 & -0.804004 & 0.000029 \\
\hline 5 & 6 & 0 & -1.397392 & -0.120158 & -0.000006 \\
\hline 6 & 1 & 0 & -1.679872 & -0.703810 & 0.882823 \\
\hline 7 & 1 & 0 & -1.679794 & -0.704599 & -0.882331 \\
\hline 8 & 1 & 0 & -1.925556 & 0.833908 & -0.000427 \\
\hline
\end{tabular}

$E=-229.0961455$ a.u.

ZPE correction $=0.061732$ a.u.

$\mathrm{H}=-229.028868$ a.u.

0 imaginary frequencies

Table S38. Acetic acid anion $\mathrm{n}=0$.

\begin{tabular}{|c|c|c|c|c|c|}
\hline \multirow{2}{*}{\multicolumn{2}{|c|}{$\begin{array}{l}\text { Center } \\
\text { Number }\end{array}$}} & \multirow{2}{*}{$\begin{array}{l}\text { Atomic } \\
\text { Number }\end{array}$} & Atomic & \multicolumn{2}{|c|}{ Coordinates (Angstroms) } \\
\hline & & & Type & $\begin{array}{ll}X & Y\end{array}$ & Z \\
\hline 1 & 6 & 0 & -0.052878 & 0.118600 & -0.000565 \\
\hline 2 & 8 & 0 & 0.560145 & 1.224790 & 0.000139 \\
\hline 3 & 8 & 0 & 0.806142 & -1.033210 & 0.000113 \\
\hline 4 & 1 & 0 & 1.683963 & -0.615621 & 0.000294 \\
\hline 5 & 1 & 0 & -1.753346 & -1.203738 & 0.000154 \\
\hline 6 & 6 & 0 & -1.403033 & -0.176372 & 0.000049 \\
\hline 7 & 1 & 0 & -2.125450 & 0.633353 & 0.000632 \\
\hline
\end{tabular}

$E=-228.4956227$ a.u.

ZPE correction $=0.047366$ a.u.

$\mathrm{H}=-228.443081$ a.u.

0 imaginary frequencies 
Table S39. Acetic acid neutral $n=1$ parallel.

\begin{tabular}{|c|c|c|c|c|c|}
\hline \multirow{2}{*}{$\begin{array}{l}\text { Center } \\
\text { Number }\end{array}$} & \multirow{2}{*}{\multicolumn{2}{|c|}{$\begin{array}{l}\text { Atomic } \\
\text { Number }\end{array}$}} & \multirow{2}{*}{$\begin{array}{l}\text { Atomic } \\
\text { Type }\end{array}$} & \multicolumn{2}{|c|}{ Coordinates (Angstroms } \\
\hline & & & & $X \quad Y$ & Z \\
\hline 1 & 6 & 0 & 1.152262 & -0.144001 & 0.000038 \\
\hline 2 & 8 & 0 & 2.084793 & -0.927674 & 0.000138 \\
\hline 3 & 8 & 0 & 1.350354 & 1.205051 & -0.000048 \\
\hline 4 & 1 & 0 & 2.315688 & 1.341379 & 0.000088 \\
\hline 5 & 6 & 0 & -0.266069 & -0.544959 & -0.000225 \\
\hline 6 & 1 & 0 & -0.418203 & -1.621212 & -0.000499 \\
\hline 7 & 6 & 0 & -1.298289 & 0.313154 & -0.000093 \\
\hline 8 & 1 & 0 & -1.086694 & 1.381526 & 0.000066 \\
\hline 9 & 6 & 0 & -2.740691 & -0.088495 & 0.000082 \\
\hline 10 & 1 & 0 & -2.864065 & -1.176198 & -0.002050 \\
\hline 11 & 1 & 0 & -3.256346 & 0.322547 & -0.878508 \\
\hline 12 & 1 & 0 & -3.254840 & 0.318743 & 0.881370 \\
\hline
\end{tabular}

$E=-306.4990475$ a.u.

ZPE correction $=0.095558$ a.u.

$\mathrm{H}=-306.39602$ a.u.

0 imaginary frequencies

Table S40. Acetic acid anion $n=1$ parallel.

\begin{tabular}{|c|c|c|c|c|c|}
\hline \multirow{2}{*}{$\begin{array}{l}\text { Center } \\
\text { Number }\end{array}$} & \multirow{2}{*}{\multicolumn{2}{|c|}{$\begin{array}{l}\text { Atomic } \\
\text { Number }\end{array}$}} & \multirow{2}{*}{$\begin{array}{l}\text { Atomic } \\
\text { Type }\end{array}$} & \multicolumn{2}{|c|}{ Coordinates (Angstron } \\
\hline & & & & $\begin{array}{ll}X & Y\end{array}$ & Z \\
\hline 1 & 6 & 0 & -1.011757 & -0.305874 & 0.000000 \\
\hline 2 & 8 & 0 & -2.257121 & -0.147022 & 0.000000 \\
\hline 3 & 8 & 0 & -0.568570 & -1.653878 & 0.000000 \\
\hline 4 & 1 & 0 & -1.409608 & -2.141255 & 0.000000 \\
\hline 5 & 6 & 0 & 0.000000 & 0.663411 & 0.000000 \\
\hline 6 & 1 & 0 & -0.347162 & 1.695113 & 0.000000 \\
\hline 7 & 6 & 0 & 1.397536 & 0.393298 & 0.000000 \\
\hline 8 & 1 & 0 & 1.663399 & -0.666354 & 0.000000 \\
\hline 9 & 1 & 0 & 3.473268 & 0.945509 & 0.000000 \\
\hline 10 & 6 & 0 & 2.439263 & 1.284424 & 0.000000 \\
\hline 11 & 1 & 0 & 2.275377 & 2.362629 & 0.000000 \\
\hline
\end{tabular}

$E=-305.9207103$ a.u.

ZPE correction $=0.081152$ a.u.

$\mathrm{H}=-305.832384$ a.u.

0 imaginary frequencies 
Table S41. Acetic acid neutral $n=1$ perpendicular.

\begin{tabular}{|c|c|c|c|c|c|}
\hline \multirow{2}{*}{\multicolumn{2}{|c|}{$\begin{array}{l}\text { Center } \\
\text { Number }\end{array}$}} & \multirow{2}{*}{$\begin{array}{l}\text { Atomic } \\
\text { Number }\end{array}$} & Atomic & \multicolumn{2}{|c|}{ Coordinates (Angstroms) } \\
\hline & & & r Type & $\begin{array}{ll}X & Y\end{array}$ & Z \\
\hline 1 & 6 & 0 & -1.137753 & -0.118243 & 0.076483 \\
\hline 2 & 8 & 0 & -1.761950 & -1.142676 & -0.107039 \\
\hline 3 & 8 & 0 & -1.707359 & 1.108600 & -0.048939 \\
\hline 4 & 1 & 0 & -2.643476 & 0.964904 & -0.290747 \\
\hline 5 & 6 & 0 & 0.296277 & -0.041429 & 0.490138 \\
\hline 6 & 1 & 0 & 0.470291 & -0.050910 & 1.565926 \\
\hline 7 & 6 & 0 & 1.310917 & 0.000127 & -0.377608 \\
\hline 8 & 1 & 0 & 1.090715 & 0.007217 & -1.446078 \\
\hline 9 & 6 & 0 & 2.762757 & 0.031535 & 0.001942 \\
\hline 10 & 1 & 0 & 3.251288 & 0.931151 & -0.395505 \\
\hline 11 & 1 & 1 & 3.295191 & -0.831170 & -0.420279 \\
\hline 12 & 1 & 0 & 2.897279 & 0.019477 & 1.088782 \\
\hline
\end{tabular}

$E=-306.4874662$ a.u.

ZPE correction $=0.094806 \mathrm{a} . \mathrm{u}$.

$H=-306.385782$ a.u.

1 imaginary frequency

Table S42. Acetic acid anion $n=1$ perpendicular.

\begin{tabular}{|c|c|c|c|c|c|}
\hline \multirow{2}{*}{$\begin{array}{l}\text { Center } \\
\text { Number }\end{array}$} & \multirow{2}{*}{\multicolumn{2}{|c|}{$\begin{array}{l}\text { Atomic } \\
\text { Number }\end{array}$}} & Atomic & \multicolumn{2}{|c|}{ Coordinates (Angstron } \\
\hline & & & Type & $X \quad Y$ & z \\
\hline 1 & 6 & 0 & -1.036641 & -0.127491 & 0.089554 \\
\hline 2 & 8 & 0 & -1.738829 & -1.115496 & -0.120346 \\
\hline 3 & 8 & 0 & -1.662721 & 1.111646 & -0.093751 \\
\hline 4 & 1 & 0 & -2.577189 & 0.897084 & -0.368941 \\
\hline 5 & 6 & 0 & 0.346552 & -0.041547 & 0.561871 \\
\hline 6 & 1 & 0 & 0.467462 & 0.015315 & 1.643881 \\
\hline 7 & 6 & 0 & 1.446228 & -0.032231 & -0.307373 \\
\hline 8 & 1 & 0 & 1.181753 & -0.100119 & -1.370738 \\
\hline 9 & 6 & 0 & 2.810789 & 0.046191 & -0.056484 \\
\hline 10 & 1 & 0 & 3.209515 & 0.122046 & 0.955616 \\
\hline 11 & 1 & 0 & 3.529287 & 0.026945 & -0.872451 \\
\hline
\end{tabular}

$E=-305.8751437$ a.u.

ZPE correction $=0.078544$ a.u.

$\mathrm{H}=-305.789477$ a.u.

1 imaginary frequency 
Table S43. Acetic acid neutral $n=2$ parallel.

\begin{tabular}{|c|c|c|c|c|c|}
\hline \multirow{2}{*}{$\begin{array}{l}\text { Center } \\
\text { Number }\end{array}$} & \multirow{2}{*}{\multicolumn{2}{|c|}{$\begin{array}{l}\text { Atomic } \\
\text { Number }\end{array}$}} & \multirow{2}{*}{$\begin{array}{l}\text { Atomic } \\
\text { Type }\end{array}$} & \multicolumn{2}{|c|}{ Coordinates (Angstroms } \\
\hline & & & & $X \quad Y$ & Z \\
\hline 1 & 6 & 0 & 2.280750 & -0.114600 & 0.000065 \\
\hline 2 & 8 & 0 & 3.280266 & -0.813497 & 0.000156 \\
\hline 3 & 8 & 0 & 2.362461 & 1.248482 & 0.000033 \\
\hline 4 & 1 & 0 & 3.312633 & 1.465322 & 0.000135 \\
\hline 5 & 6 & 0 & 0.907599 & -0.632796 & -0.000096 \\
\hline 6 & 1 & 0 & 0.843957 & -1.717460 & -0.000120 \\
\hline 7 & 6 & 0 & -0.201528 & 0.140055 & -0.000175 \\
\hline 8 & 1 & 0 & -0.085426 & 1.222775 & -0.000146 \\
\hline 9 & 6 & 0 & -1.554849 & -0.378259 & -0.000248 \\
\hline 10 & 1 & 0 & -1.673967 & -1.462622 & -0.000381 \\
\hline 11 & 6 & 0 & -2.648850 & 0.409991 & -0.000163 \\
\hline 12 & 1 & 0 & -2.507895 & 1.492576 & -0.000169 \\
\hline 13 & 6 & 0 & -4.065515 & -0.075360 & 0.000238 \\
\hline 14 & 1 & 0 & -4.121300 & -1.169105 & -0.002181 \\
\hline 15 & 1 & 0 & -4.608797 & 0.299325 & -0.878373 \\
\hline 16 & 1 & 0 & -4.606670 & 0.295128 & 0.881996 \\
\hline
\end{tabular}

$E=-383.9081125$ a.u.

ZPE correction $=0.129154$ a.u.

$\mathrm{H}=-383.76934$ a.u.

0 imaginary frequencies

Table S44. Acetic acid anion $n=2$ parallel.

\begin{tabular}{|c|c|c|c|c|c|}
\hline \multirow{2}{*}{$\begin{array}{l}\text { Center } \\
\text { Number }\end{array}$} & \multirow{2}{*}{\multicolumn{2}{|c|}{$\begin{array}{l}\text { Atomic } \\
\text { Number }\end{array}$}} & \multirow{2}{*}{$\begin{array}{l}\text { Atomic } \\
\text { Type }\end{array}$} & \multicolumn{2}{|c|}{ Coordinates (Angstror } \\
\hline & & & & $X \quad Y$ & Z \\
\hline 1 & 6 & 0 & 2.181793 & -0.120317 & -0.000021 \\
\hline 2 & 8 & 0 & 3.282387 & -0.710841 & 0.000004 \\
\hline 3 & 8 & 0 & 2.225876 & 1.287702 & 0.000009 \\
\hline 4 & 1 & 0 & 3.180669 & 1.470985 & 0.000017 \\
\hline 5 & 6 & 0 & 0.888285 & -0.685206 & -0.000002 \\
\hline 6 & 1 & 0 & 0.863438 & -1.772759 & 0.000008 \\
\hline 7 & 6 & 0 & -0.315957 & 0.043829 & -0.000003 \\
\hline 8 & 1 & 0 & -0.214892 & 1.131688 & -0.000012 \\
\hline 9 & 6 & 0 & -1.609867 & -0.453889 & 0.000005 \\
\hline 10 & 1 & 0 & -1.759344 & -1.536764 & 0.000014 \\
\hline 11 & 6 & 0 & -2.773729 & 0.376425 & -0.000003 \\
\hline 12 & 1 & 0 & -2.570976 & 1.453050 & -0.000014 \\
\hline 13 & 1 & 0 & -4.888573 & 0.732957 & -0.000011 \\
\hline 14 & 6 & 0 & -4.086538 & -0.001386 & 0.000002 \\
\hline 15 & 1 & 0 & -4.380345 & -1.050775 & 0.000019 \\
\hline
\end{tabular}

$E=-383.3429429$ a.u.

ZPE correction $=0.114677$ a.u.

$\mathrm{H}=-383.218972$ a.u.

0 imaginary frequencies 
Table S45. Acetic acid neutral $n=2$ perpendicular.

\begin{tabular}{|c|c|c|c|c|c|}
\hline \multirow{2}{*}{$\begin{array}{l}\text { Center } \\
\text { Number }\end{array}$} & \multirow{2}{*}{\multicolumn{2}{|c|}{$\begin{array}{l}\text { Atomic } \\
\text { Number }\end{array}$}} & \multirow{2}{*}{$\begin{array}{l}\text { Atomic } \\
\text { Type }\end{array}$} & \multicolumn{2}{|c|}{ Coordinates (Angstroms) } \\
\hline & & & & $X \quad Y$ & Z \\
\hline 1 & 6 & 0 & -2.270987 & -0.111851 & 0.046934 \\
\hline 2 & 8 & 0 & -2.889913 & -1.115491 & -0.240740 \\
\hline 3 & 8 & 0 & -2.807979 & 1.129708 & -0.076976 \\
\hline 4 & 1 & 0 & -3.718859 & 1.014653 & -0.412514 \\
\hline 5 & 6 & 0 & -0.882688 & -0.080843 & 0.594214 \\
\hline 6 & 1 & 0 & -0.806896 & -0.145284 & 1.678991 \\
\hline 7 & 6 & 0 & 0.214536 & -0.019423 & -0.179166 \\
\hline 8 & 1 & 0 & 0.098911 & 0.042349 & -1.261922 \\
\hline 9 & 6 & 0 & 1.578077 & -0.032118 & 0.329232 \\
\hline 10 & 1 & 0 & 1.698984 & -0.099171 & 1.411779 \\
\hline 11 & 6 & 0 & 2.669662 & 0.034987 & -0.455132 \\
\hline 12 & 1 & 0 & 2.530632 & 0.101142 & -1.536107 \\
\hline 13 & 6 & 0 & 4.087558 & 0.025910 & 0.032048 \\
\hline 14 & 1 & 0 & 4.617498 & 0.937199 & -0.278329 \\
\hline 15 & 1 & 0 & 4.645287 & -0.820152 & -0.392960 \\
\hline 16 & 1 & 0 & 4.140627 & -0.044438 & 1.124006 \\
\hline $\begin{array}{l}E=-383 . \\
Z P E \text { corre } \\
H=-383 . \\
1 \text { imagin }\end{array}$ & $\begin{array}{l}.89 \\
\text { ecti } \\
.75\end{array}$ & $\begin{array}{l}49586 \text { a.u } \\
\text { tion }=0.12 \\
7678 \text { a.u. } \\
\text { y frequen }\end{array}$ & 8208 a.u. & & \\
\hline
\end{tabular}


Table S46. Acetic acid anion $\mathrm{n}=2$ perpendicular.

\begin{tabular}{|c|c|c|c|c|c|}
\hline \multirow{2}{*}{$\begin{array}{l}\text { Center } \\
\text { Number }\end{array}$} & \multirow{2}{*}{\multicolumn{2}{|c|}{$\begin{array}{l}\text { Atomic } \\
\text { Number }\end{array}$}} & \multirow{2}{*}{$\begin{array}{l}\text { Atomic } \\
\text { Type }\end{array}$} & \multicolumn{2}{|c|}{ Coordinates (Angstroms) } \\
\hline & & & & $\begin{array}{ll}X & Y\end{array}$ & Z \\
\hline 1 & 6 & 0 & -2.176675 & -0.119236 & 0.038097 \\
\hline 2 & 8 & 0 & -2.842698 & -1.093738 & -0.294447 \\
\hline 3 & 8 & 0 & -2.741704 & 1.132887 & -0.171564 \\
\hline 4 & 1 & 0 & -3.614741 & 0.957844 & -0.576295 \\
\hline 5 & 6 & 0 & -0.855733 & -0.077334 & 0.683997 \\
\hline 6 & 1 & 0 & -0.869739 & -0.070440 & 1.773536 \\
\hline 7 & 6 & 0 & 0.331882 & -0.048314 & -0.029535 \\
\hline 8 & 1 & 0 & 0.230183 & -0.056120 & -1.121451 \\
\hline 9 & 6 & 0 & 1.656322 & -0.012590 & 0.438749 \\
\hline 10 & 1 & 0 & 1.827714 & 0.000289 & 1.518525 \\
\hline 11 & 6 & 0 & 2.785257 & 0.007879 & -0.420678 \\
\hline 12 & 1 & 0 & 2.542006 & -0.004871 & -1.490771 \\
\hline 13 & 6 & 0 & 4.125341 & 0.041408 & -0.111550 \\
\hline 14 & 1 & 0 & 4.476869 & 0.054604 & 0.920214 \\
\hline 15 & 1 & 0 & 4.884553 & 0.054625 & -0.890149 \\
\hline
\end{tabular}

$E=-383.3045775$ a.u.

ZPE correction $=0.112552$ a.u.

$\mathrm{H}=-383.183008$ a.u.

1 imaginary frequency

Table S47. Acetic acid neutral $n=3$ parallel.

\begin{tabular}{|c|c|c|c|c|c|}
\hline \multirow{2}{*}{$\begin{array}{l}\text { Center } \\
\text { Number }\end{array}$} & \multirow{2}{*}{\multicolumn{2}{|c|}{$\begin{array}{l}\text { Atomic } \\
\text { Number }\end{array}$}} & \multirow{2}{*}{$\begin{array}{l}\text { Atomic } \\
\text { Type }\end{array}$} & \multicolumn{2}{|c|}{ Coordinates (Angstron } \\
\hline & & & & $X \quad Y$ & Z \\
\hline 1 & 6 & 0 & 3.447430 & -0.073311 & 0.000064 \\
\hline 2 & 8 & 0 & 4.477311 & -0.727868 & 0.000225 \\
\hline 3 & 8 & 0 & 3.471197 & 1.292818 & -0.000002 \\
\hline 4 & 1 & 0 & 4.411504 & 1.548796 & 0.000130 \\
\hline 5 & 6 & 0 & 2.099703 & -0.649440 & -0.000070 \\
\hline 6 & 1 & 0 & 2.082216 & -1.735768 & -0.000101 \\
\hline 7 & 6 & 0 & 0.955053 & 0.075596 & -0.000105 \\
\hline 8 & 1 & 0 & 1.025985 & 1.162286 & -0.000069 \\
\hline 9 & 6 & 0 & -0.367793 & -0.497673 & -0.000175 \\
\hline 10 & 1 & 0 & -0.443780 & -1.585562 & -0.000229 \\
\hline 11 & 6 & 0 & -1.503500 & 0.247860 & -0.000154 \\
\hline 12 & 1 & 0 & -1.414350 & 1.335635 & -0.000104 \\
\hline 13 & 6 & 0 & -2.844182 & -0.295702 & -0.000162 \\
\hline 14 & 1 & 0 & -2.936713 & -1.383101 & -0.000277 \\
\hline 15 & 6 & 0 & -3.962347 & 0.459896 & -0.000043 \\
\hline 16 & 1 & 0 & -3.855135 & 1.546424 & -0.000020 \\
\hline 17 & 6 & 0 & -5.363692 & -0.069321 & 0.000253 \\
\hline 18 & 1 & 0 & -5.384485 & -1.164483 & -0.001413 \\
\hline 19 & 1 & 0 & -5.919439 & 0.285787 & -0.878908 \\
\hline 20 & 1 & 0 & -5.917900 & 0.282960 & 0.881558 \\
\hline
\end{tabular}

$E=-461.318061$ a.u.

ZPE correction $=0.162648$ a.u.

$\mathrm{H}=-461.143604$ a.u.

0 imaginary frequencies 
Table $\mathrm{S} .48$ Acetic acid anion $\mathrm{n}=3$ parallel.

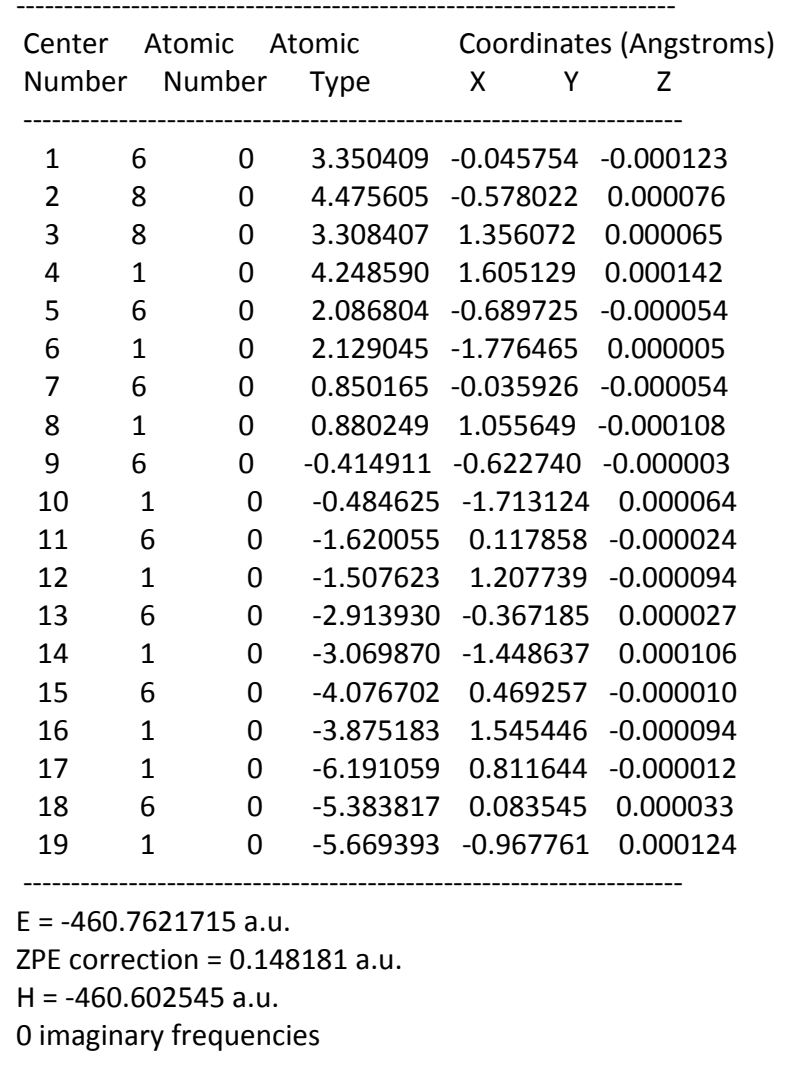


Table S49. Acetic acid neutral $n=3$ perpendicular.

\begin{tabular}{|c|c|c|c|c|c|}
\hline \multirow{2}{*}{$\begin{array}{l}\text { Center } \\
\text { Number }\end{array}$} & \multirow{2}{*}{\multicolumn{2}{|c|}{$\begin{array}{l}\text { Atomic } \\
\text { Number }\end{array}$}} & \multirow{2}{*}{$\begin{array}{l}\text { Atomic } \\
\text { Type }\end{array}$} & \multicolumn{2}{|c|}{ Coordinates (Angstroms } \\
\hline & & & & $X \quad Y$ & Z \\
\hline 1 & 6 & 0 & -3.440826 & -0.098680 & -0.026672 \\
\hline 2 & 8 & 0 & -4.053541 & -0.946042 & -0.643051 \\
\hline 3 & 8 & 0 & -3.962514 & 1.129551 & 0.227939 \\
\hline 4 & 1 & 0 & -4.856507 & 1.147625 & -0.166933 \\
\hline 5 & 6 & 0 & -2.079701 & -0.273935 & 0.558328 \\
\hline 6 & 1 & 0 & -2.053823 & -0.683462 & 1.567164 \\
\hline 7 & 6 & 0 & -0.945725 & 0.005009 & -0.111496 \\
\hline 8 & 1 & 0 & -1.013658 & 0.411982 & -1.120982 \\
\hline 9 & 6 & 0 & 0.387675 & -0.197094 & 0.417311 \\
\hline 10 & 1 & 0 & 0.464509 & -0.607645 & 1.424959 \\
\hline 11 & 6 & 0 & 1.520687 & 0.094697 & -0.266958 \\
\hline 12 & 1 & 0 & 1.433797 & 0.504312 & -1.274899 \\
\hline 13 & 6 & 0 & 2.863147 & -0.098243 & 0.243294 \\
\hline 14 & 1 & 0 & 2.952012 & -0.507467 & 1.251324 \\
\hline 15 & 6 & 0 & 3.984445 & 0.194120 & -0.446448 \\
\hline 16 & 1 & 0 & 3.883654 & 0.602643 & -1.453893 \\
\hline 17 & 6 & 0 & 5.383458 & 0.005324 & 0.057228 \\
\hline 18 & 1 & 0 & 5.395954 & -0.412444 & 1.069845 \\
\hline 19 & 1 & 0 & 5.930709 & 0.958365 & 0.074370 \\
\hline 20 & 1 & 0 & 5.952825 & -0.669166 & -0.597586 \\
\hline
\end{tabular}

$E=-461.3043873$ a.u.

ZPE correction $=0.161647$ a.u.

$\mathrm{H}=-461.131453$ a.u.

1 imaginary frequency 
Table S50. Acetic acid anion $\mathrm{n}=3$ perpendicular.

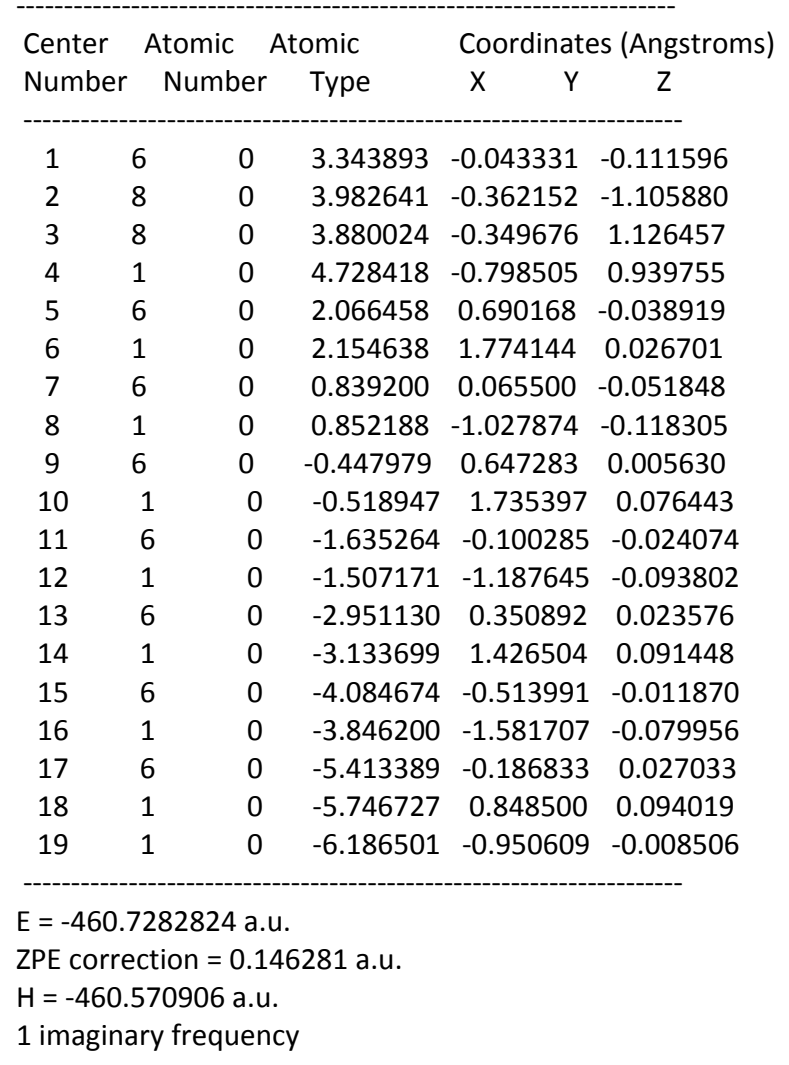


Table S51. Acetic acid neutral $n=4$ parallel.

\begin{tabular}{|c|c|c|c|c|c|}
\hline \multirow{2}{*}{$\begin{array}{l}\text { Center } \\
\text { Number }\end{array}$} & \multirow{2}{*}{\multicolumn{2}{|c|}{$\begin{array}{c}\text { Atomic } \\
\text { Number }\end{array}$}} & \multirow{2}{*}{$\begin{array}{l}\text { Atomic } \\
\text { Type }\end{array}$} & \multicolumn{2}{|c|}{ Coordinates (Angstroms) } \\
\hline & & & & $X \quad Y$ & Z \\
\hline 1 & 6 & 0 & 4.634828 & -0.028965 & 0.000075 \\
\hline 2 & 8 & 0 & 5.682905 & -0.654688 & 0.000272 \\
\hline 3 & 8 & 0 & 4.621499 & 1.337727 & -0.000056 \\
\hline 4 & 1 & 0 & 5.554662 & 1.618420 & 0.000093 \\
\hline 5 & 6 & 0 & 3.304283 & -0.641379 & -0.000067 \\
\hline 6 & 1 & 0 & 3.316142 & -1.727762 & -0.000108 \\
\hline 7 & 6 & 0 & 2.138810 & 0.052383 & -0.000072 \\
\hline 8 & 1 & 0 & 2.180488 & 1.140616 & -0.000025 \\
\hline 9 & 6 & 0 & 0.834547 & -0.555724 & -0.000128 \\
\hline 10 & 1 & 0 & 0.787097 & -1.645197 & -0.000189 \\
\hline 11 & 6 & 0 & -0.324395 & 0.160002 & -0.000098 \\
\hline 12 & 1 & 0 & -0.262480 & 1.249722 & -0.000041 \\
\hline 13 & 6 & 0 & -1.642209 & -0.415963 & -0.000132 \\
\hline 14 & 1 & 0 & -1.710081 & -1.504814 & -0.000202 \\
\hline 15 & 6 & 0 & -2.788908 & 0.315566 & -0.000070 \\
\hline 16 & 1 & 0 & -2.714900 & 1.404560 & -0.000003 \\
\hline 17 & 6 & 0 & -4.120872 & -0.246843 & -0.000071 \\
\hline 18 & 1 & 0 & -4.196210 & -1.335784 & -0.000172 \\
\hline 19 & 6 & 0 & -5.252868 & 0.488784 & 0.000034 \\
\hline 20 & 1 & 0 & -5.165472 & 1.577101 & 0.000081 \\
\hline 21 & 6 & 0 & -6.644388 & -0.066392 & 0.000194 \\
\hline 22 & 1 & 0 & -6.644274 & -1.161858 & -0.000794 \\
\hline 23 & 1 & 0 & -7.207038 & 0.276760 & -0.879450 \\
\hline 24 & 1 & 0 & -7.206128 & 0.275107 & 0.881085 \\
\hline $\begin{array}{l}\text { ZPE corre } \\
\mathrm{H}=-538 \\
0 \text { imagin }\end{array}$ & 3.51 & y frequenci & 32 a.u. & & \\
\hline
\end{tabular}


Table S52. Acetic acid anion $n=4$ parallel.

\begin{tabular}{|c|c|c|c|c|c|}
\hline \multirow{2}{*}{$\begin{array}{l}\text { Center } \\
\text { Number }\end{array}$} & \multirow{2}{*}{\multicolumn{2}{|c|}{$\begin{array}{l}\text { Atomic } \\
\text { Number }\end{array}$}} & \multirow{2}{*}{$\begin{array}{l}\text { Atomic } \\
\text { Type }\end{array}$} & \multicolumn{2}{|c|}{ Coordinates (Angstroms) } \\
\hline & & & & $X \quad Y$ & Z \\
\hline 1 & 6 & 0 & 4.535341 & 0.042918 & -0.000354 \\
\hline 2 & 8 & 0 & 5.675923 & -0.447841 & 0.000314 \\
\hline 3 & 8 & 0 & 4.431730 & 1.436822 & 0.000202 \\
\hline 4 & 1 & 0 & 5.358232 & 1.733072 & 0.000535 \\
\hline 5 & 6 & 0 & 3.295664 & -0.656507 & -0.000181 \\
\hline 6 & 1 & 0 & 3.385326 & -1.740136 & 0.000026 \\
\hline 7 & 6 & 0 & 2.038409 & -0.056440 & -0.000205 \\
\hline 8 & 1 & 0 & 2.018469 & 1.034959 & -0.000395 \\
\hline 9 & 6 & 0 & 0.797467 & -0.704110 & -0.000051 \\
\hline 10 & 1 & 0 & 0.781273 & -1.796308 & 0.000142 \\
\hline 11 & 6 & 0 & -0.433583 & -0.023614 & -0.000128 \\
\hline 12 & 1 & 0 & -0.378013 & 1.070063 & -0.000327 \\
\hline 13 & 6 & 0 & -1.707058 & -0.579765 & 0.000029 \\
\hline 14 & 1 & 0 & -1.798565 & -1.668277 & 0.000235 \\
\hline 15 & 6 & 0 & -2.902090 & 0.183087 & -0.000056 \\
\hline 16 & 1 & 0 & -2.774651 & 1.270860 & -0.000267 \\
\hline 17 & 6 & 0 & -4.197340 & -0.288792 & 0.000091 \\
\hline 18 & 1 & 0 & -4.363848 & -1.368419 & 0.000295 \\
\hline 19 & 6 & 0 & -5.355029 & 0.558513 & -0.000014 \\
\hline 20 & 1 & 0 & -5.147957 & 1.633079 & -0.000219 \\
\hline 21 & 1 & 0 & -7.467170 & 0.901675 & 0.000024 \\
\hline 22 & 6 & 0 & -6.659951 & 0.173858 & 0.000119 \\
\hline 23 & 1 & 0 & -6.945310 & -0.877305 & 0.000323 \\
\hline $\begin{array}{l}\mathrm{H}=-537 . \\
0 \text { imagin }\end{array}$ & .98 & $\begin{array}{l}34252 \text { a.u. } \\
\text { y frequen }\end{array}$ & 74 a.u. & & \\
\hline
\end{tabular}


Table S53. Acetic acid neutral $n=4$ perpendicular.

\begin{tabular}{|c|c|c|c|c|c|}
\hline \multirow{2}{*}{\multicolumn{2}{|c|}{$\begin{array}{l}\text { Center } \\
\text { Number }\end{array}$}} & \multirow{2}{*}{$\begin{array}{l}\text { Atomic } \\
\text { Number }\end{array}$} & \multirow{2}{*}{$\begin{array}{l}\text { Atomic } \\
\text { Type }\end{array}$} & \multicolumn{2}{|c|}{ Coordinates (Angstroms) } \\
\hline & & & & $\begin{array}{ll}X & Y\end{array}$ & Z \\
\hline 1 & 6 & 0 & -4.631426 & -0.000620 & 0.097946 \\
\hline 2 & 8 & 0 & -5.236916 & -0.006529 & 1.149964 \\
\hline 3 & 8 & 0 & -5.140478 & -0.555083 & -1.032797 \\
\hline 4 & 1 & 0 & -6.022883 & -0.909047 & -0.805919 \\
\hline 5 & 6 & 0 & -3.286973 & 0.608795 & -0.117847 \\
\hline 6 & 1 & 0 & -3.287299 & 1.669039 & -0.366934 \\
\hline 7 & 6 & 0 & -2.134311 & -0.068097 & 0.050572 \\
\hline 8 & 1 & 0 & -2.175362 & -1.126075 & 0.312218 \\
\hline 9 & 6 & 0 & -0.817453 & 0.513321 & -0.092274 \\
\hline 10 & 1 & 0 & -0.767513 & 1.573869 & -0.342268 \\
\hline 11 & 6 & 0 & 0.336970 & -0.185173 & 0.065918 \\
\hline 12 & 1 & 0 & 0.275862 & -1.245560 & 0.317518 \\
\hline 13 & 6 & 0 & 1.657995 & 0.374035 & -0.072995 \\
\hline 14 & 1 & 0 & 1.723327 & 1.435565 & -0.317086 \\
\hline 15 & 6 & 0 & 2.806391 & -0.335715 & 0.080624 \\
\hline 16 & 1 & 0 & 2.738112 & -1.397273 & 0.325229 \\
\hline 17 & 6 & 0 & 4.136812 & 0.216916 & -0.057707 \\
\hline 18 & 1 & 0 & 4.204017 & 1.277720 & -0.306750 \\
\hline 19 & 6 & 0 & 5.274926 & -0.490708 & 0.100439 \\
\hline 20 & 1 & 0 & 5.198399 & -1.551171 & 0.348640 \\
\hline 21 & 6 & 0 & 6.661618 & 0.061786 & -0.036487 \\
\hline 22 & 1 & 0 & 7.225818 & -0.046001 & 0.900729 \\
\hline 23 & 1 & 0 & 6.649203 & 1.123654 & -0.305746 \\
\hline 24 & 1 & 0 & 7.230174 & -0.479070 & -0.806103 \\
\hline
\end{tabular}


Table S54. Acetic acid anion $n=4$ perpendicular.

\begin{tabular}{|c|c|c|c|c|c|}
\hline \multirow{2}{*}{$\begin{array}{l}\text { Center } \\
\text { Number }\end{array}$} & \multicolumn{2}{|c|}{ Atomic } & Atomic & \multicolumn{2}{|c|}{ Coordinates (Angstroms } \\
\hline & & Number & r Type & $\begin{array}{ll}X & Y\end{array}$ & Z \\
\hline 1 & 6 & 0 & 4.527044 & -0.140576 & -0.107574 \\
\hline 2 & 8 & 0 & 5.145078 & -0.486414 & -1.104010 \\
\hline 3 & 8 & 0 & 5.036836 & -0.472604 & 1.130141 \\
\hline 4 & 1 & 0 & 5.861807 & -0.965946 & 0.951933 \\
\hline 5 & 6 & 0 & 3.280981 & 0.650616 & -0.039047 \\
\hline 6 & 1 & 0 & 3.416032 & 1.729827 & 0.022745 \\
\hline 7 & 6 & 0 & 2.032154 & 0.083402 & -0.051176 \\
\hline 8 & 1 & 0 & 1.988440 & -1.008815 & -0.109332 \\
\hline 9 & 6 & 0 & 0.774440 & 0.738523 & -0.000807 \\
\hline 10 & 1 & 0 & 0.767104 & 1.829126 & 0.059892 \\
\hline 11 & 6 & 0 & -0.445386 & 0.062354 & -0.025683 \\
\hline 12 & 1 & 0 & -0.387443 & -1.030630 & -0.085427 \\
\hline 13 & 6 & 5 & -1.735915 & 0.604122 & 0.016310 \\
\hline 14 & 1 & 0 & -1.838549 & 1.690397 & 0.074519 \\
\hline 15 & 6 & 0 & -2.914038 & -0.171422 & -0.012472 \\
\hline 16 & 1 & 0 & -2.767645 & -1.256121 & -0.072269 \\
\hline 17 & 6 & 5 & -4.227387 & 0.267674 & 0.024778 \\
\hline 18 & 1 & 1 & -4.419910 & 1.341647 & 0.084786 \\
\hline 19 & 6 & 5 & -5.358543 & -0.606936 & -0.011646 \\
\hline 20 & 1 & 0 & -5.117683 & -1.673617 & -0.072420 \\
\hline 21 & 1 & 0 & -7.459042 & -1.032642 & -0.014969 \\
\hline 22 & 6 & 5 & -6.681613 & -0.273580 & 0.019433 \\
\hline 23 & 1 & 1 & -7.008842 & 0.763850 & 0.078794 \\
\hline
\end{tabular}

$E=-538.1487257$ a.u.

ZPE correction $=0.179968$ a.u.

$\mathrm{H}=-537.955547$ a.u.

1 imaginary frequency 
4. Cartesian coordinates, absolute energies, zero-point energy corrections, absolute enthalpies, and number of imaginary frequencies for acetyl fluoride, the acetyl fluoride enolate anion, and their $n=1-4$ vinylogues

Table S55. Acetyl fluoride neutral $n=0$.

\begin{tabular}{|c|c|c|c|c|c|}
\hline \multirow{2}{*}{$\begin{array}{l}\text { Center } \\
\text { Number }\end{array}$} & \multirow{2}{*}{\multicolumn{2}{|c|}{$\begin{array}{l}\text { Atomic } \\
\text { Number }\end{array}$}} & Atomic & \multicolumn{2}{|c|}{ Coordinates (Angstroms) } \\
\hline & & & Type & $\begin{array}{ll}X & Y\end{array}$ & Z \\
\hline 1 & 6 & 0 & -1.075386 & -0.854936 & 0.000000 \\
\hline 2 & 1 & 0 & -0.967392 & -1.494607 & 0.882521 \\
\hline 3 & 1 & 0 & -0.967392 & -1.494607 & -0.882521 \\
\hline 4 & 1 & 0 & -2.055981 & -0.377497 & 0.000000 \\
\hline 5 & 6 & 0 & 0.000000 & 0.188397 & 0.000000 \\
\hline 6 & 8 & 0 & -0.091491 & 1.375031 & 0.000000 \\
\hline 7 & 9 & 0 & 1.241668 & -0.403812 & 0.000000 \\
\hline
\end{tabular}

$E=-253.10986$ a.u.

ZPE correction $=0.049019 \mathrm{a} . \mathrm{u}$.

$\mathrm{H}=-253.055577$ a.u.

0 imaginary frequencies

Table S56. Acetyl fluoride anion $\mathrm{n}=0$.

\begin{tabular}{cccccc}
\multicolumn{2}{c}{ Center } & \multicolumn{2}{c}{ Atomic } & Atomic & \multicolumn{2}{c}{ Coordinates (Angstroms) } \\
Number & Number & Type & X & $Y$ & $Z$ \\
-1 & 6 & 0 & 0.000000 & 0.180651 & 0.000000 \\
2 & 8 & 0 & -0.828534 & 1.093392 & 0.000000 \\
3 & 9 & 0 & -0.605382 & -1.171416 & 0.000000 \\
4 & 6 & 0 & 1.373158 & 0.094518 & 0.000000 \\
5 & 1 & 0 & 1.958810 & 1.008689 & 0.000000 \\
6 & 1 & 0 & 1.878951 & -0.864092 & 0.000000
\end{tabular}

$E=-252.5278779$ a.u.

ZPE correction $=0.034864$ a.u.

$\mathrm{H}=-252.488071$ a.u.

0 imaginary frequencies 
Table S57. Acetyl fluoride neutral $n=1$ parallel.

\begin{tabular}{|c|c|c|c|c|c|}
\hline \multirow{2}{*}{$\begin{array}{l}\text { Center } \\
\text { Number }\end{array}$} & \multirow{2}{*}{\multicolumn{2}{|c|}{$\begin{array}{l}\text { Atomic } \\
\text { Number }\end{array}$}} & \multirow{2}{*}{$\begin{array}{l}\text { Atomic } \\
\text { Type }\end{array}$} & \multicolumn{2}{|c|}{ Coordinates (Angstroms) } \\
\hline & & & & $X \quad Y$ & Z \\
\hline 1 & 6 & 0 & 1.136793 & -0.314879 & 0.000000 \\
\hline 2 & 8 & 0 & 2.300882 & -0.039948 & 0.000000 \\
\hline 3 & 9 & 0 & 0.752591 & -1.638544 & 0.000000 \\
\hline 4 & 6 & 0 & 0.000000 & 0.609371 & 0.000000 \\
\hline 5 & 1 & 0 & 0.284505 & 1.658357 & 0.000000 \\
\hline 6 & 6 & 0 & -1.287602 & 0.224770 & 0.000000 \\
\hline 7 & 1 & 0 & -1.513715 & -0.840248 & 0.000000 \\
\hline 8 & 6 & 0 & -2.454416 & 1.159945 & 0.000000 \\
\hline 9 & 1 & 0 & -3.087816 & 0.981881 & 0.879541 \\
\hline 10 & 1 & 0 & -2.144185 & 2.209373 & 0.000000 \\
\hline 11 & 1 & 0 & -3.087816 & 0.981881 & -0.879541 \\
\hline
\end{tabular}

$E=-330.5138806$ a.u.

ZPE correction $=0.082792$ a.u.

$\mathrm{H}=-330.423797$ a.u.

0 imaginary frequencies

Table S58. Acetyl fluoride anion $n=1$ parallel.

\begin{tabular}{cccccc}
\multicolumn{2}{c}{ Center } & \multicolumn{2}{c}{ Atomic } & Atomic & \multicolumn{3}{c}{ Coordinates (Angstroms) } \\
Number & Number & Type & X & $Y$ & $Z$ \\
\hline 1 & 6 & 0 & -1.062354 & -0.247066 & 0.000000 \\
2 & 8 & 0 & -2.280984 & -0.105128 & 0.000000 \\
3 & 9 & 0 & -0.617560 & -1.630628 & 0.000000 \\
4 & 6 & 0 & 0.000000 & 0.655798 & 0.000000 \\
5 & 1 & 0 & -0.295618 & 1.703840 & 0.000000 \\
6 & 6 & 0 & 1.387792 & 0.325178 & 0.000000 \\
7 & 1 & 0 & 1.611905 & -0.742801 & 0.000000 \\
8 & 6 & 0 & 2.455373 & 1.180200 & 0.000000 \\
9 & 1 & 0 & 2.326818 & 2.263017 & 0.000000 \\
10 & 1 & 0 & 3.477948 & 0.807959 & 0.000000
\end{tabular}

$E=-329.9500493$ a.u.

ZPE correction $=0.068623 \mathrm{a} . \mathrm{u}$.

$\mathrm{H}=-329.874476$ a.u.

0 imaginary frequencies 
Table S59. Acetyl fluoride neutral $n=1$ perpendicular.

\begin{tabular}{|c|c|c|c|c|c|}
\hline \multirow{2}{*}{$\begin{array}{l}\text { Center } \\
\text { Number }\end{array}$} & \multirow{2}{*}{\multicolumn{2}{|c|}{$\begin{array}{l}\text { Atomic } \\
\text { Number }\end{array}$}} & Atomic & \multicolumn{2}{|c|}{ Coordinates (Angstroms } \\
\hline & & & Type & $X \quad Y$ & Z \\
\hline 1 & 6 & 0 & -1.147336 & -0.134552 & 0.068788 \\
\hline 2 & 8 & 0 & -1.825950 & -1.099858 & -0.092412 \\
\hline 3 & 9 & 0 & -1.697420 & 1.115899 & -0.091739 \\
\hline 4 & 6 & 0 & 0.275591 & -0.047490 & 0.488602 \\
\hline 5 & 1 & 0 & 0.435701 & 0.019451 & 1.564249 \\
\hline 6 & 6 & 0 & 1.294560 & -0.049901 & -0.376181 \\
\hline 7 & 1 & 0 & 1.081287 & -0.113294 & -1.443910 \\
\hline 8 & 6 & 0 & 2.742225 & 0.020634 & 0.010021 \\
\hline 9 & 1 & 0 & 3.220018 & 0.901156 & -0.439137 \\
\hline 10 & 1 & 0 & 2.871138 & 0.074918 & 1.095924 \\
\hline 11 & 1 & 0 & 3.285998 & -0.858607 & -0.359559 \\
\hline
\end{tabular}

$E=-330.5010431$ a.u.

ZPE correction $=0.081926$ a.u.

$\mathrm{H}=-330.412356$ a.u.

1 imaginary frequency

Table S60. Acetyl fluoride anion $\mathrm{n}=1$ perpendicular.

\begin{tabular}{|c|c|c|c|c|c|}
\hline \multirow{2}{*}{\multicolumn{2}{|c|}{$\begin{array}{l}\text { Center } \\
\text { Number }\end{array}$}} & & \multirow{2}{*}{$\begin{array}{l}\text { Atomic } \\
\text { Type }\end{array}$} & \multicolumn{2}{|c|}{ Coordinates (Angstroms) } \\
\hline & & & & $\begin{array}{ll}X & Y\end{array}$ & Z \\
\hline 1 & 6 & 0 & -1.034330 & -0.176846 & 0.102534 \\
\hline 2 & 8 & 0 & -1.784887 & -1.078289 & -0.163077 \\
\hline 3 & 9 & 0 & -1.665514 & 1.135694 & -0.103177 \\
\hline 4 & 6 & 0 & 0.326818 & -0.091894 & 0.571020 \\
\hline 5 & 1 & 0 & 0.455043 & -0.071891 & 1.652578 \\
\hline 6 & 6 & 0 & 1.423628 & -0.037985 & -0.310266 \\
\hline 7 & 1 & 0 & 1.155059 & -0.069684 & -1.373048 \\
\hline 8 & 6 & 0 & 2.780750 & 0.041689 & -0.053502 \\
\hline 9 & 1 & 0 & 3.175290 & 0.085261 & 0.961841 \\
\hline 10 & 1 & 0 & 3.502130 & 0.051598 & -0.866864 \\
\hline
\end{tabular}

$E=-329.9027055$ a.u.

ZPE correction $=0.06571$ a.u.

$\mathrm{H}=-329.829942$ a.u.

1 imaginary frequency 
Table S61. Acetyl fluoride neutral $n=2$ parallel.

\begin{tabular}{|c|c|c|c|c|c|}
\hline \multirow{2}{*}{$\begin{array}{l}\text { Center } \\
\text { Number }\end{array}$} & \multicolumn{2}{|c|}{ Atomic } & Atomic & \multicolumn{2}{|c|}{ Coordinates (Angstroms) } \\
\hline & & Number & Type & $\begin{array}{ll}X & Y\end{array}$ & Z \\
\hline 1 & 6 & 0 & 1.194059 & -1.959363 & 0.000000 \\
\hline 2 & 8 & 0 & 1.264175 & -3.155219 & 0.000000 \\
\hline 3 & 9 & 0 & 2.356938 & -1.214101 & 0.000000 \\
\hline 4 & 6 & 0 & -0.007317 & -1.133077 & 0.000000 \\
\hline 5 & 1 & 0 & -0.935504 & -1.697950 & 0.000000 \\
\hline 6 & 6 & 0 & 0.000000 & 0.220703 & 0.000000 \\
\hline 7 & 1 & 0 & 0.957536 & 0.738510 & 0.000000 \\
\hline 8 & 6 & 0 & -1.191847 & 1.041072 & 0.000000 \\
\hline 9 & 1 & 0 & -2.154726 & 0.528957 & 0.000000 \\
\hline 10 & 6 & 0 & -1.153865 & 2.389770 & 0.000000 \\
\hline 11 & 1 & 0 & -0.178597 & 2.880392 & 0.000000 \\
\hline 12 & 6 & 0 & -2.348593 & 3.290221 & 0.000000 \\
\hline 13 & 1 & 0 & -2.341206 & 3.948508 & 0.879885 \\
\hline 14 & 1 & 0 & -2.341206 & 3.948508 & -0.879885 \\
\hline 15 & 1 & 0 & -3.286763 & 2.725782 & 0.000000 \\
\hline
\end{tabular}

$E=-407.9233939$ a.u.

ZPE correction $=0.116299$ a.u.

$\mathrm{H}=-407.797620$ a.u.

0 imaginary frequencies

Table S62. Acetyl fluoride anion $n=2$ parallel.

\begin{tabular}{|c|c|c|c|c|c|}
\hline \multirow{2}{*}{$\begin{array}{l}\text { Center } \\
\text { Number }\end{array}$} & \multirow{2}{*}{\multicolumn{2}{|c|}{$\begin{array}{l}\text { Atomic } \\
\text { Number }\end{array}$}} & \multirow{2}{*}{$\begin{array}{l}\text { Atomic } \\
r \quad \text { Type }\end{array}$} & \multicolumn{2}{|c|}{ Coordinates (Angstrom } \\
\hline & & & & $\begin{array}{ll}X & Y\end{array}$ & Z \\
\hline 1 & 6 & 0 & -2.204456 & -0.140135 & -0.000188 \\
\hline 2 & 8 & 0 & -3.310915 & -0.660054 & 0.000040 \\
\hline 3 & 9 & 0 & -2.204263 & 1.299128 & 0.000112 \\
\hline 4 & 6 & 0 & -0.909613 & -0.681277 & -0.000115 \\
\hline 5 & 1 & 0 & & -1.769005 & -0.000228 \\
\hline 6 & 6 & 0 & 0.297022 & 0.051491 & 0.000071 \\
\hline 7 & 1 & 0 & 0.198589 & 1.138612 & 0.000143 \\
\hline 8 & 6 & 0 & 1.583383 & -0.454605 & 0.000110 \\
\hline 9 & 1 & 0 & 1.725992 & -1.538220 & 0.000328 \\
\hline 10 & 6 & 0 & 2.755842 & 0.367939 & -0.000127 \\
\hline 11 & 1 & 0 & 2.564165 & 1.446125 & -0.000339 \\
\hline 12 & 6 & 0 & 4.061107 & -0.027358 & -0.000038 \\
\hline 13 & 1 & 0 & 4.339637 & -1.080847 & 0.000618 \\
\hline 14 & 1 & 0 & 4.873898 & 0.695273 & -0.000124 \\
\hline
\end{tabular}

$E=-407.3705568$ a.u.

ZPE correction $=0.102163 \mathrm{a} . \mathrm{u}$.

$H=-407.259326$ a.u.

0 imaginary frequencies 
Table S63. Acetyl fluoride neutral $n=2$ perpendicular.

\begin{tabular}{|c|c|c|c|c|c|}
\hline \multirow{2}{*}{$\begin{array}{l}\text { Center } \\
\text { Number }\end{array}$} & \multirow{2}{*}{\multicolumn{2}{|c|}{$\begin{array}{l}\text { Atomic } \\
\text { Number }\end{array}$}} & \multirow{2}{*}{$\begin{array}{l}\text { Atomic } \\
\text { Type }\end{array}$} & \multicolumn{2}{|c|}{ Coordinates (Angstroms) } \\
\hline & & & & $X \quad Y$ & Z \\
\hline 1 & 6 & 0 & -2.274062 & -0.126393 & 0.044377 \\
\hline 2 & 8 & 0 & -2.945348 & -1.085572 & -0.175949 \\
\hline 3 & 9 & 0 & -2.792364 & 1.128383 & -0.182874 \\
\hline 4 & 6 & 0 & -0.898868 & -0.051175 & 0.596061 \\
\hline 5 & 1 & 0 & -0.836761 & 0.020804 & 1.680958 \\
\hline 6 & 6 & 0 & 0.202418 & -0.069450 & -0.175577 \\
\hline 7 & 1 & 0 & 0.091850 & -0.135706 & -1.258377 \\
\hline 8 & 6 & 0 & 1.562059 & -0.010074 & 0.336532 \\
\hline 9 & 1 & 0 & 1.680439 & 0.051434 & 1.419358 \\
\hline 10 & 6 & 0 & 2.653572 & -0.025367 & -0.451101 \\
\hline 11 & 1 & 0 & 2.514323 & -0.087138 & -1.532211 \\
\hline 12 & 6 & 0 & 4.070406 & 0.036334 & 0.032464 \\
\hline 13 & 1 & 0 & 4.590904 & 0.908694 & -0.386347 \\
\hline 14 & 1 & 0 & 4.125191 & 0.096779 & 1.124712 \\
\hline 15 & 1 & 0 & 4.634969 & -0.848998 & -0.291171 \\
\hline $\begin{array}{l}E=-407 . \\
Z P E \text { corre } \\
H=-407 . \\
1 \text { imagin }\end{array}$ & $\begin{array}{l}908 \\
\text { ecti } \\
.78\end{array}$ & $\begin{array}{l}86485 \text { a.u } \\
\text { ion }=0.11 \\
34358 \text { a.u. } \\
\text { frequenc }\end{array}$ & 5335 a.u. & & \\
\hline
\end{tabular}


Table S64. Acetyl fluoride anion $\mathrm{n}=2$ perpendicular.

\begin{tabular}{|c|c|c|c|c|c|}
\hline \multirow{2}{*}{$\begin{array}{l}\text { Center } \\
\text { Number }\end{array}$} & \multirow{2}{*}{\multicolumn{2}{|c|}{$\begin{array}{l}\text { Atomic } \\
\text { Number }\end{array}$}} & \multirow{2}{*}{$\begin{array}{l}\text { Atomic } \\
\text { Type }\end{array}$} & \multicolumn{2}{|c|}{ Coordinates (Angstroms } \\
\hline & & & & $X \quad Y$ & Z \\
\hline 1 & 6 & 0 & -2.174600 & -0.157118 & 0.040983 \\
\hline 2 & 8 & 0 & -2.881691 & -1.047331 & -0.344903 \\
\hline 3 & 9 & 0 & -2.730789 & 1.153855 & -0.177468 \\
\hline 4 & 6 & 0 & -0.871990 & -0.124198 & 0.683896 \\
\hline 5 & 1 & 0 & -0.884760 & -0.150811 & 1.772727 \\
\hline 6 & 6 & 0 & 0.317400 & -0.062767 & -0.035224 \\
\hline 7 & 1 & 0 & 0.213675 & -0.039688 & -1.126023 \\
\hline 8 & 6 & 0 & 1.634712 & -0.031362 & 0.436273 \\
\hline 9 & 1 & 0 & 1.805289 & -0.044487 & 1.515890 \\
\hline 10 & & 0 & 2.768207 & 0.013733 & -0.421677 \\
\hline 11 & 1 & 0 & 2.529753 & 0.026506 & -1.492286 \\
\hline 12 & 6 & 0 & 4.102004 & 0.043354 & -0.100486 \\
\hline 13 & 1 & 0 & 4.443719 & 0.032576 & 0.934472 \\
\hline 14 & 1 & 0 & 4.868548 & 0.080005 & -0.870926 \\
\hline
\end{tabular}

$E=-407.3293465$ a.u.

ZPE correction $=0.099682$ a.u.

$\mathrm{H}=-407.220718$ a.u.

1 imaginary frequency

Table S65. Acetyl fluoride neutral $n=3$ parallel.

\begin{tabular}{|c|c|c|c|c|c|}
\hline \multirow{2}{*}{$\begin{array}{l}\text { Center } \\
\text { Number }\end{array}$} & \multirow{2}{*}{\multicolumn{2}{|c|}{$\begin{array}{l}\text { Atomic } \\
\text { Number }\end{array}$}} & Atomic & \multicolumn{2}{|c|}{ Coordinates (Angstron } \\
\hline & & & r Type & $X \quad Y$ & Z \\
\hline 1 & 6 & 0 & -2.906124 & -1.866255 & 0.000000 \\
\hline 2 & 8 & 0 & -4.098925 & -1.983571 & 0.000000 \\
\hline 3 & 9 & 0 & -2.117140 & -3.002438 & 0.000000 \\
\hline 4 & 6 & 0 & -2.126370 & -0.637229 & 0.000000 \\
\hline 5 & 1 & 0 & -2.724684 & 0.269599 & 0.000000 \\
\hline 6 & 6 & 0 & -0.769761 & -0.592016 & 0.000000 \\
\hline 7 & 1 & 0 & -0.216972 & -1.529774 & 0.000000 \\
\hline 8 & 6 & 0 & 0.000000 & 0.623809 & 0.000000 \\
\hline 9 & 1 & 0 & -0.545290 & 1.567829 & 0.000000 \\
\hline 10 & 6 & 5 & 1.359647 & 0.638052 & 0.000000 \\
\hline 11 & 1 & 1 & 1.891245 & -0.315047 & 0.000000 \\
\hline 12 & 6 & 5 & 2.169961 & 1.834898 & 0.000000 \\
\hline 13 & 1 & 1 & 1.643821 & 2.790712 & 0.000000 \\
\hline 14 & 6 & 5 & 3.519946 & 1.824062 & 0.000000 \\
\hline 15 & 1 & 1 & 4.031438 & 0.859546 & 0.000000 \\
\hline 16 & 6 & 5 & 4.394736 & 3.039017 & 0.000000 \\
\hline 17 & 1 & 1 & 5.052900 & 3.048935 & 0.880051 \\
\hline 18 & 1 & 1 & 5.052900 & 3.048935 & -0.880051 \\
\hline 19 & 1 & 1 & 3.808083 & 3.963746 & 0.000000 \\
\hline
\end{tabular}

$E=-485.3336168$ a.u.

ZPE correction $=0.149778$ a.u.

$H=-485.172167$ a.u.

0 imaginary frequencies 
Table S.66 Acetyl fluoride anion $\mathrm{n}=3$ parallel.

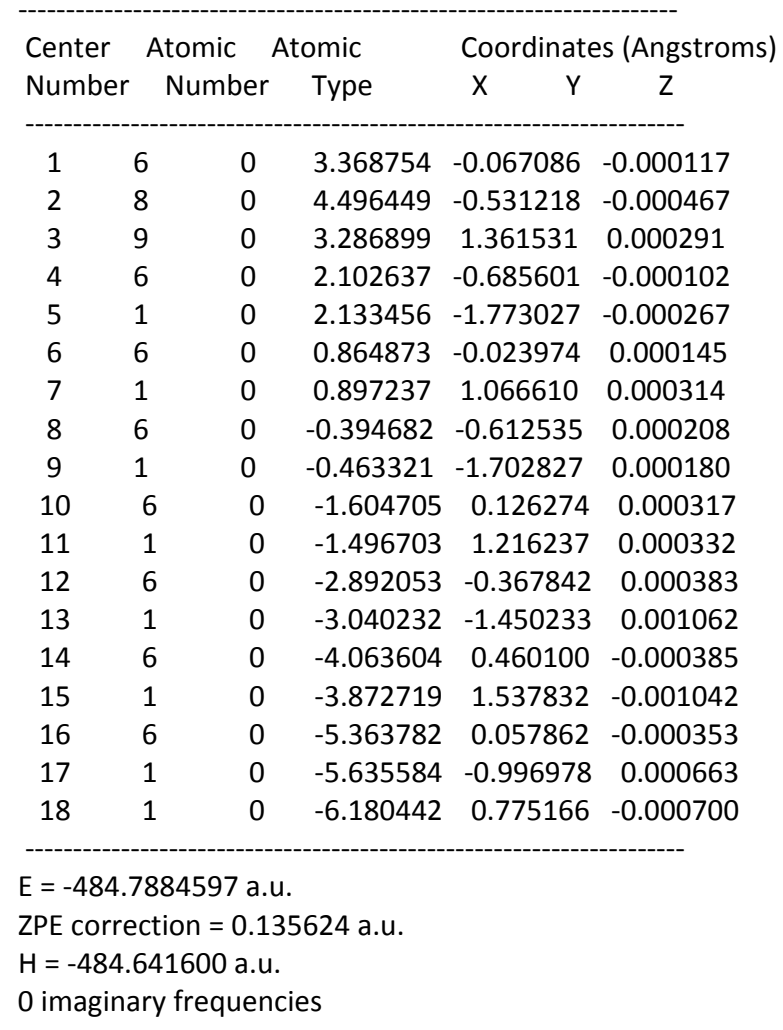


Table S67. Acetyl fluoride neutral $n=3$ perpendicular.

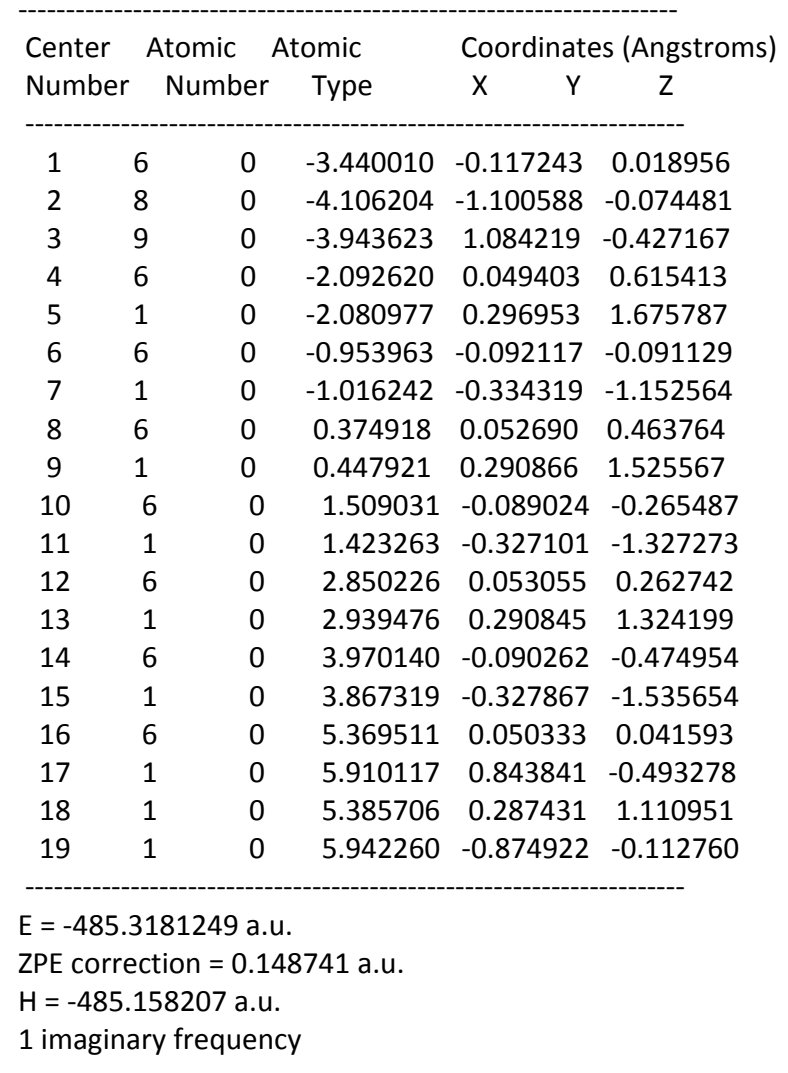


Table S68. Acetyl fluoride anion $\mathrm{n}=3$ perpendicular.

\begin{tabular}{|c|c|c|c|c|c|}
\hline \multirow{2}{*}{$\begin{array}{l}\text { Center } \\
\text { Number }\end{array}$} & \multirow{2}{*}{\multicolumn{2}{|c|}{$\begin{array}{c}\text { Atomic } \\
\text { Number }\end{array}$}} & \multirow{2}{*}{$\begin{array}{l}\text { Atomic } \\
\text { Type }\end{array}$} & \multicolumn{2}{|c|}{ Coordinates (Angstroms) } \\
\hline & & & & $X \quad Y$ & Z \\
\hline 1 & 6 & 0 & 3.340814 & -0.030173 & -0.144457 \\
\hline 2 & 8 & 0 & 4.015844 & -0.355808 & -1.080753 \\
\hline 3 & 9 & 0 & 3.857915 & -0.423762 & 1.123868 \\
\hline 4 & 6 & 0 & 2.080174 & 0.697437 & -0.041519 \\
\hline 5 & 1 & 0 & 2.168532 & 1.778643 & 0.053109 \\
\hline 6 & 6 & 0 & 0.850882 & 0.065249 & -0.061811 \\
\hline 7 & 1 & 0 & 0.867304 & -1.025292 & -0.157940 \\
\hline 8 & 6 & 0 & -0.430741 & 0.643243 & 0.017186 \\
\hline 9 & 1 & 0 & -0.503467 & 1.728543 & 0.118889 \\
\hline 10 & 6 & 0 & -1.620742 & -0.105361 & -0.033216 \\
\hline 11 & 1 & 0 & -1.493456 & -1.189785 & -0.136056 \\
\hline 12 & 6 & 0 & -2.931251 & 0.348173 & 0.030725 \\
\hline 13 & 1 & 0 & -3.110572 & 1.421385 & 0.133176 \\
\hline 14 & 6 & 0 & -4.070257 & -0.511971 & -0.029692 \\
\hline 15 & 1 & 0 & -3.838321 & -1.577844 & -0.133156 \\
\hline 16 & 6 & 0 & -5.393464 & -0.174485 & 0.023211 \\
\hline 17 & 1 & 0 & -5.716489 & 0.861105 & 0.125318 \\
\hline 18 & 1 & 0 & -6.174004 & -0.929113 & -0.034686 \\
\hline $\begin{array}{l}E=-484 . \\
Z P E \text { corre } \\
H=-484 . \\
1 \text { imagin }\end{array}$ & $\begin{array}{l}.75 \\
\text { rect } \\
.65 \\
\text { hary }\end{array}$ & $\begin{array}{l}12811 \text { a.u } \\
\text { tion }=0.13 \\
4388 \text { a.u. } \\
\text { y frequen }\end{array}$ & 3343 a.u. & & \\
\hline
\end{tabular}


Table S69. Acetyl fluoride neutral $n=4$ parallel.

\begin{tabular}{|c|c|c|c|c|c|}
\hline \multirow{2}{*}{$\begin{array}{l}\text { Center } \\
\text { Number }\end{array}$} & \multirow{2}{*}{\multicolumn{2}{|c|}{$\begin{array}{l}\text { Atomic } \\
\text { Number }\end{array}$}} & \multirow{2}{*}{$\begin{array}{l}\text { Atomic } \\
\text { Type }\end{array}$} & \multicolumn{2}{|c|}{ Coordinates (Angstroms) } \\
\hline & & & & $\begin{array}{ll}X & Y\end{array}$ & Z \\
\hline 1 & 6 & 0 & -1.834608 & -4.257541 & 0.000000 \\
\hline 2 & 8 & 0 & -1.775583 & -5.455087 & 0.000000 \\
\hline 3 & 9 & 0 & -3.075785 & -3.644797 & 0.000000 \\
\hline 4 & 6 & 0 & -0.735946 & -3.304790 & 0.000000 \\
\hline 5 & 1 & 0 & 0.250061 & -3.760848 & 0.000000 \\
\hline 6 & 6 & 0 & -0.893169 & -1.954906 & 0.000000 \\
\hline 7 & 1 & 0 & -1.903352 & -1.549314 & 0.000000 \\
\hline 8 & 6 & 0 & 0.191036 & -1.013269 & 0.000000 \\
\hline 9 & 1 & 0 & 1.206963 & -1.408670 & 0.000000 \\
\hline 10 & 6 & 0 & 0.000000 & 0.336893 & 0.000000 \\
\hline 11 & 1 & 0 & -1.023566 & 0.715692 & 0.000000 \\
\hline 12 & 6 & 0 & 1.053511 & 1.313467 & 0.000000 \\
\hline 13 & 1 & 0 & 2.079496 & 0.943315 & 0.000000 \\
\hline 14 & 6 & 0 & 0.835357 & 2.656831 & 0.000000 \\
\hline 15 & 1 & 0 & -0.194250 & 3.018991 & 0.000000 \\
\hline 16 & 6 & 0 & 1.877662 & 3.657493 & 0.000000 \\
\hline 17 & 1 & 0 & 2.908286 & 3.298579 & 0.000000 \\
\hline 18 & 6 & 0 & 1.644844 & 4.987648 & 0.000000 \\
\hline 19 & 1 & 0 & 0.609380 & 5.333729 & 0.000000 \\
\hline 20 & 6 & 0 & 2.700622 & 6.049711 & 0.000000 \\
\hline 21 & 1 & 0 & 2.604592 & 6.700978 & 0.880128 \\
\hline 22 & 1 & 0 & 3.708672 & 5.621222 & 0.000000 \\
\hline 23 & 1 & 0 & 2.604592 & 6.700978 & -0.880128 \\
\hline $\begin{array}{l}H=-562 . \\
0 \text { imagin }\end{array}$ & .54 & $\begin{array}{l}1697 \text { a.u. } \\
\text { y frequenc }\end{array}$ & 320 a.u. & & \\
\hline
\end{tabular}


Table S70. Acetyl fluoride anion $n=4$ parallel.

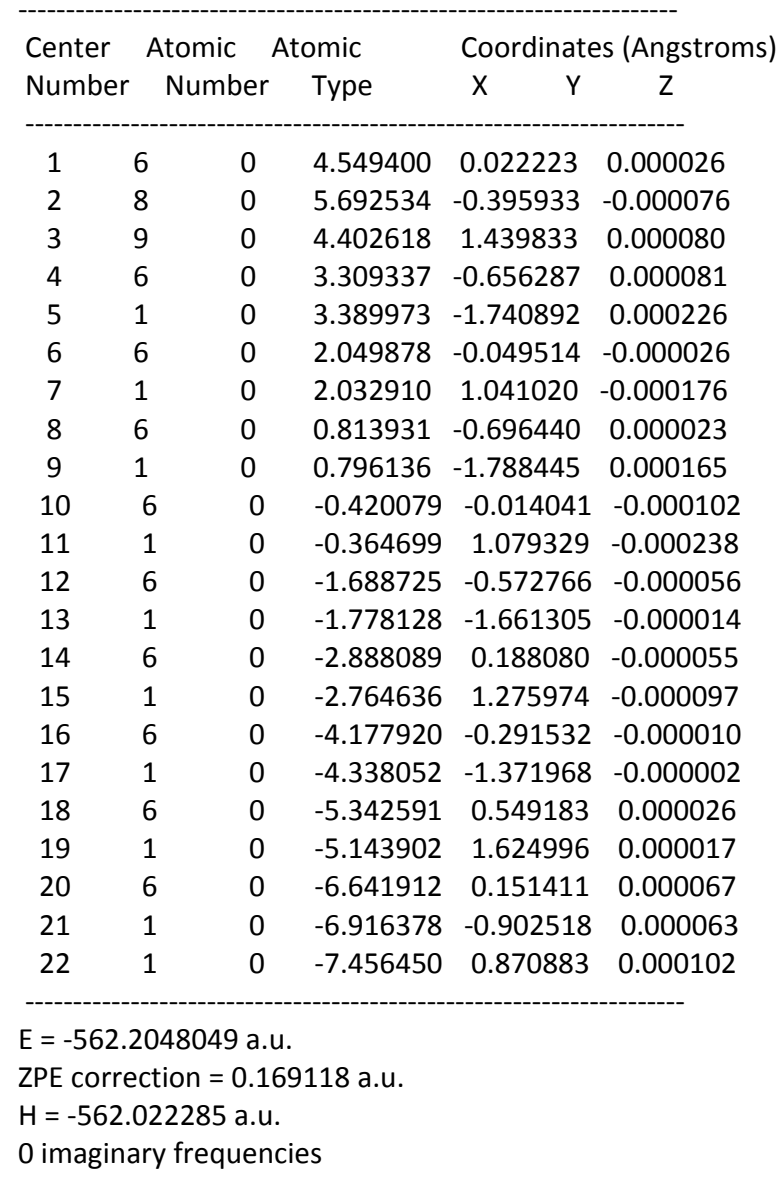


Table S71. Acetyl fluoride neutral $n=4$ perpendicular.

\begin{tabular}{|c|c|c|c|c|c|}
\hline \multirow{2}{*}{$\begin{array}{l}\text { Center } \\
\text { Number }\end{array}$} & \multirow{2}{*}{\multicolumn{2}{|c|}{$\begin{array}{l}\text { Atomic } \\
\text { Number }\end{array}$}} & \multirow{2}{*}{$\begin{array}{l}\text { Atomic } \\
\text { Type }\end{array}$} & \multicolumn{2}{|c|}{ Coordinates (Angstroms) } \\
\hline & & & & $X \quad Y$ & Z \\
\hline 1 & 6 & 0 & 4.627195 & -0.053539 & -0.107567 \\
\hline 2 & 8 & 0 & 5.289690 & -0.387188 & -1.040036 \\
\hline 3 & 9 & 0 & 5.117380 & -0.233416 & 1.166840 \\
\hline 4 & 6 & 0 & 3.299109 & 0.605219 & -0.088340 \\
\hline 5 & 1 & 0 & 3.319650 & 1.693924 & -0.087119 \\
\hline 6 & 6 & 0 & 2.138626 & -0.081614 & -0.073283 \\
\hline 7 & 1 & 0 & 2.169897 & -1.171711 & -0.068484 \\
\hline 8 & 6 & 0 & 0.828481 & 0.528157 & -0.064991 \\
\hline 9 & 1 & 0 & 0.784713 & 1.617806 & -0.074235 \\
\hline 10 & 6 & 0 & -0.328738 & -0.184210 & -0.043085 \\
\hline 11 & 1 & 0 & -0.270049 & -1.274175 & -0.033683 \\
\hline 12 & 6 & 0 & -1.647911 & 0.394034 & -0.030991 \\
\hline 13 & 1 & 0 & -1.713737 & 1.483067 & -0.040008 \\
\hline 14 & 6 & 0 & -2.794945 & -0.334531 & -0.008904 \\
\hline 15 & 1 & 0 & -2.723464 & -1.423705 & 0.000039 \\
\hline 16 & 6 & 0 & -4.126675 & 0.230570 & 0.004667 \\
\hline 17 & 1 & 0 & -4.199532 & 1.319733 & -0.003827 \\
\hline 18 & 6 & 0 & -5.260084 & -0.501778 & 0.027487 \\
\hline 19 & 1 & 0 & -5.175697 & -1.590324 & 0.035846 \\
\hline 20 & 6 & 0 & -6.650326 & 0.057216 & 0.043025 \\
\hline 21 & 1 & 0 & -7.200209 & -0.274601 & 0.935032 \\
\hline 22 & 1 & 0 & -6.647071 & 1.152693 & 0.032412 \\
\hline 23 & 1 & 0 & -7.226833 & -0.291614 & -0.825351 \\
\hline $\begin{array}{l}H=-562 \\
1 \text { imagin }\end{array}$ & .53 & $\begin{array}{l}32672 \text { a.u. } \\
\text { y frequenc }\end{array}$ & 74 a.u. & & \\
\hline
\end{tabular}


Table S72. Acetyl fluoride anion $n=4$ perpendicular.

\begin{tabular}{|c|c|c|c|c|c|}
\hline \multirow{2}{*}{$\begin{array}{l}\text { Center } \\
\text { Number }\end{array}$} & \multirow{2}{*}{\multicolumn{2}{|c|}{$\begin{array}{l}\text { Atomic } \\
\text { Number }\end{array}$}} & \multirow{2}{*}{$\begin{array}{l}\text { Atomic } \\
\text { Type }\end{array}$} & \multicolumn{2}{|c|}{ Coordinates (Angstroms) } \\
\hline & & & & $X \quad Y$ & Z \\
\hline 1 & 6 & 0 & 4.520867 & -0.157261 & -0.126262 \\
\hline 2 & 8 & 0 & 5.184065 & -0.579812 & -1.030439 \\
\hline 3 & 9 & 0 & 5.046853 & -0.390525 & 1.168477 \\
\hline 4 & 6 & 0 & 3.288152 & 0.629440 & -0.096229 \\
\hline 5 & 1 & 0 & 3.429296 & 1.709186 & -0.086094 \\
\hline 6 & 6 & 0 & 2.034510 & 0.060767 & -0.081567 \\
\hline 7 & 1 & 0 & 1.990510 & -1.032506 & -0.093092 \\
\hline 8 & 6 & 0 & 0.783842 & 0.718845 & -0.054017 \\
\hline 9 & 1 & 0 & 0.777453 & 1.810800 & -0.036172 \\
\hline 10 & 6 & 0 & -0.440493 & 0.043065 & -0.045852 \\
\hline 11 & 1 & 0 & -0.385227 & -1.051312 & -0.061854 \\
\hline 12 & 6 & 0 & -1.724581 & 0.591248 & -0.019342 \\
\hline 13 & 1 & 0 & -1.822898 & 1.679213 & -0.003515 \\
\hline 14 & 6 & 0 & -2.908774 & -0.180253 & -0.011355 \\
\hline 15 & 1 & 0 & -2.769041 & -1.267027 & -0.028094 \\
\hline 16 & 6 & 0 & -4.215567 & 0.270697 & 0.015133 \\
\hline 17 & 1 & 0 & -4.399308 & 1.347587 & 0.032704 \\
\hline 18 & 6 & 0 & -5.355836 & -0.595650 & 0.021112 \\
\hline 19 & 1 & 0 & -5.126208 & -1.666048 & 0.003096 \\
\hline 20 & 6 & 0 & -6.672599 & -0.244486 & 0.046213 \\
\hline 21 & 1 & 0 & -6.985877 & 0.798671 & 0.064975 \\
\hline 22 & 1 & 0 & -7.460028 & -0.993822 & 0.048258 \\
\hline $\begin{array}{l}\text { ZPE corre } \\
\mathrm{H}=-561 . \\
1 \text { imagin }\end{array}$ & & y frequen & 74 a.u. & & \\
\hline
\end{tabular}


5. Cartesian coordinates, absolute energies, zero-point energy corrections, absolute enthalpies, and number of imaginary frequencies for methylpropane, the methylpropyl anion, and their $n=1-4$ vinylogues

Table S73. Methylpropane neutral $n=0$.

\begin{tabular}{|c|c|c|c|c|c|}
\hline \multirow{2}{*}{$\begin{array}{l}\text { Center } \\
\text { Number }\end{array}$} & \multicolumn{2}{|r|}{ Atomic } & Atomic & \multicolumn{2}{|c|}{ Coordinates (Angstroms } \\
\hline & & Number & Type & $\begin{array}{ll}X & Y\end{array}$ & Z \\
\hline 1 & 6 & 0 & 0.000000 & 1.463124 & -0.095884 \\
\hline 2 & 1 & 0 & 0.000000 & 1.522101 & -1.193093 \\
\hline 3 & 1 & 0 & 0.886629 & 1.998970 & 0.265969 \\
\hline 4 & 1 & 0 & -0.886629 & 1.998970 & 0.265969 \\
\hline 5 & 6 & 0 & 0.000000 & 0.000000 & 0.372609 \\
\hline 6 & 1 & 0 & 0.000000 & 0.000000 & 1.473725 \\
\hline 7 & 6 & 0 & -1.267103 & -0.731562 & -0.095884 \\
\hline 8 & 1 & 0 & -1.318178 & -0.761051 & -1.193093 \\
\hline 9 & 1 & 0 & -2.174473 & -0.231642 & 0.265969 \\
\hline 10 & 1 & 0 & -1.287844 & -1.767328 & 0.265969 \\
\hline 11 & 6 & 0 & 1.267103 & -0.731562 & -0.095884 \\
\hline 12 & 1 & 0 & 1.318178 & -0.761051 & -1.193093 \\
\hline 13 & 1 & 0 & 1.287844 & -1.767328 & 0.265969 \\
\hline 14 & 1 & 0 & 2.174473 & -0.231642 & 0.265969 \\
\hline
\end{tabular}

$E=-158.4641151$ a.u.

ZPE correction $=0.131915$ a.u.

$H=-158.325565$ a.u.

0 imaginary frequencies

Table S74. Methylpropane anion $n=0$.

\begin{tabular}{|c|c|c|c|c|c|}
\hline \multirow{2}{*}{\multicolumn{2}{|c|}{$\begin{array}{l}\text { Center } \\
\text { Number }\end{array}$}} & Atomic & tomic & \multicolumn{2}{|c|}{ Coordinates (Angstroms) } \\
\hline & & Number & Type & $\begin{array}{ll}X & Y\end{array}$ & Z \\
\hline 1 & 6 & 0 & 0.028982 & 0.089335 & -0.364941 \\
\hline 2 & 1 & 0 & 0.042878 & 0.033185 & -1.471564 \\
\hline 3 & 6 & 0 & 0.887839 & -1.128776 & 0.115713 \\
\hline 4 & 1 & 0 & 0.889806 & -1.179348 & 1.215631 \\
\hline 5 & 1 & 0 & 1.930029 & -1.005773 & -0.204089 \\
\hline 6 & 1 & 0 & 0.521166 & -2.104381 & -0.274884 \\
\hline 7 & 6 & 0 & -1.430344 & -0.154803 & 0.074910 \\
\hline 8 & 1 & 0 & -1.508681 & -0.074838 & 1.170854 \\
\hline 9 & 1 & 0 & -1.800165 & -1.154124 & -0.211781 \\
\hline 10 & 1 & 0 & -2.088577 & 0.608846 & -0.355393 \\
\hline 11 & 6 & 0 & 0.513213 & 1.462986 & 0.055126 \\
\hline 12 & 1 & 0 & 1.535485 & 1.671657 & -0.307411 \\
\hline 13 & 1 & 0 & 0.479924 & 1.592321 & 1.153793 \\
\hline $\begin{array}{l}E=-157 \\
Z P E \text { corr } \\
H=-157 \\
0 \text { imagin }\end{array}$ & $\begin{array}{l}.78 \\
\text { rect } \\
.66\end{array}$ & $\begin{array}{l}889596 \text { a.u } \\
\text { tion }=0.11 \\
67495 \text { a.u. } \\
y \text { frequenc }\end{array}$ & 4788 a.u. & & \\
\hline
\end{tabular}


Table S75. Methylpropane neutral $n=1$.

\begin{tabular}{|c|c|c|c|c|c|}
\hline \multirow{2}{*}{$\begin{array}{l}\text { Center } \\
\text { Number }\end{array}$} & \multirow{2}{*}{\multicolumn{2}{|c|}{$\begin{array}{l}\text { Atomic } \\
\text { Number }\end{array}$}} & Atomic & \multicolumn{2}{|c|}{ Coordinates (Angstroms) } \\
\hline & & & T Type & $X \quad Y$ & Z \\
\hline 1 & 6 & 0 & 1.015326 & 0.451437 & -0.041082 \\
\hline 2 & 1 & 0 & 1.132119 & 1.103667 & -0.923056 \\
\hline 3 & 6 & 0 & 1.765884 & 1.136213 & 1.120578 \\
\hline 4 & 1 & 0 & 1.709225 & 0.527000 & 2.031547 \\
\hline 5 & 1 & 0 & 1.337271 & 2.119853 & 1.348991 \\
\hline 6 & 1 & 0 & 2.824775 & 1.280375 & 0.871889 \\
\hline 7 & 6 & 0 & 1.650643 & -0.902912 & -0.380861 \\
\hline 8 & 1 & 0 & 1.587126 & -1.596423 & 0.467515 \\
\hline 9 & 1 & 0 & 2.711755 & -0.775251 & -0.625875 \\
\hline 10 & 1 & 0 & 1.163095 & -1.375287 & -1.241721 \\
\hline 11 & 6 & 5 & -0.466111 & 0.388741 & 0.262350 \\
\hline 12 & 1 & 1 & -0.922621 & 1.357517 & 0.484437 \\
\hline 13 & 6 & 5 & -1.249387 & -0.696424 & 0.296665 \\
\hline 14 & 1 & 1 & -0.820762 & -1.675380 & 0.080050 \\
\hline 15 & 6 & 0 & -2.719773 & -0.684975 & 0.615660 \\
\hline 16 & 1 & 0 & -3.311162 & -1.092289 & -0.216274 \\
\hline 17 & 1 & 0 & -2.941887 & -1.308045 & 1.493240 \\
\hline 18 & 1 & 1 & -3.079882 & 0.329589 & 0.821590 \\
\hline
\end{tabular}

$E=-235.8620705$ a.u.

ZPE correction $=0.165263$ a.u.

$\mathrm{H}=-235.687936$ a.u.

0 imaginary frequencies

Table S76. Methylpropane anion n=1.

\begin{tabular}{ccccccc}
\multicolumn{2}{c}{ Center } & \multicolumn{2}{c}{ Atomic } & \multicolumn{2}{c}{ Atomic } & \multicolumn{3}{c}{ Coordinates (Angstroms) } \\
Number & \multicolumn{2}{c}{ Number } & Type & X & $Y$ & $Z$ \\
\hline 1 & 6 & 0 & -0.962618 & -0.019822 & -0.359798 \\
2 & 1 & 0 & -1.069045 & 0.055216 & -1.473555 \\
3 & 6 & 0 & -2.060095 & -1.003763 & 0.088423 \\
4 & 1 & 0 & -2.072187 & -1.080395 & 1.185559 \\
5 & 1 & 0 & -1.871407 & -2.009895 & -0.311682 \\
6 & 1 & 0 & -3.057769 & -0.683617 & -0.252294 \\
7 & 6 & 0 & -1.312308 & 1.386719 & 0.174739 \\
8 & 1 & 0 & -1.333479 & 1.377422 & 1.273914 \\
9 & 1 & 0 & -2.296194 & 1.721684 & -0.199334 \\
10 & 1 & 0 & -0.569050 & 2.131508 & -0.133778 \\
11 & 6 & 0 & 0.397637 & -0.512595 & 0.064645 \\
12 & 1 & 0 & 0.481126 & -1.594275 & 0.212234 \\
13 & 6 & 0 & 1.584339 & 0.217320 & -0.072695 \\
14 & 1 & 0 & 1.451315 & 1.277107 & -0.331724 \\
15 & 6 & 0 & 2.921069 & -0.164549 & 0.058005 \\
16 & 1 & 0 & 3.204966 & -1.180172 & 0.338008 \\
17 & 1 & 0 & 3.723579 & 0.565552 & -0.027256
\end{tabular}

$E=-235.227548$ a.u.

ZPE correction $=0.148006$ a.u.

$\mathrm{H}=-235.070399$ a.u.

0 imaginary frequencies 
Table S77. Methylpropane neutral $n=2$.

\begin{tabular}{|c|c|c|c|c|c|}
\hline \multirow{2}{*}{\multicolumn{2}{|c|}{$\begin{array}{l}\text { Center } \\
\text { Number }\end{array}$}} & & Atomic & \multicolumn{2}{|c|}{ Coordinates (Angstroms } \\
\hline & & & r Type & $X \quad Y$ & Z \\
\hline 1 & 6 & 0 & -2.232731 & -0.064193 & -0.381216 \\
\hline 2 & 1 & 0 & -2.625412 & -0.178392 & -1.406092 \\
\hline 3 & 6 & 0 & -3.065331 & -1.002537 & 0.519177 \\
\hline 4 & 1 & 0 & -2.741134 & -0.924607 & 1.564523 \\
\hline 5 & 1 & 0 & -2.958263 & -2.050243 & 0.212505 \\
\hline 6 & 1 & 0 & -4.130771 & -0.744970 & 0.471569 \\
\hline 7 & 6 & 0 & -2.425656 & 1.401741 & 0.027733 \\
\hline 8 & 1 & 0 & -2.074024 & 1.578638 & 1.052294 \\
\hline 9 & 1 & 0 & -3.487942 & 1.670563 & -0.009289 \\
\hline 10 & 1 & 0 & -1.884662 & 2.083318 & -0.639075 \\
\hline 11 & 6 & 0 & -0.792313 & -0.518398 & -0.413656 \\
\hline 12 & 1 & 0 & -0.649308 & -1.554807 & -0.730487 \\
\hline 13 & 6 & 0 & 0.301825 & 0.197545 & -0.091973 \\
\hline 14 & 1 & 1 & 0.196940 & 1.233963 & 0.229122 \\
\hline 15 & 6 & 5 & 1.662493 & -0.322501 & -0.144267 \\
\hline 16 & 1 & 1 & 1.775332 & -1.359968 & -0.466677 \\
\hline 17 & 6 & 5 & 2.763350 & 0.383900 & 0.173507 \\
\hline 18 & 1 & 1 & 2.643047 & 1.420582 & 0.495398 \\
\hline 19 & 6 & 0 & 4.170165 & -0.137074 & 0.124573 \\
\hline 20 & 1 & 1 & 4.650161 & -0.083897 & 1.112155 \\
\hline 21 & 1 & 1 & 4.793105 & 0.458662 & -0.557686 \\
\hline 22 & 1 & 0 & 4.202119 & -1.179742 & -0.211524 \\
\hline
\end{tabular}

$E=-313.2702$ a.u.

ZPE correction $=0.19871 \mathrm{a} . \mathrm{u}$.

$\mathrm{H}=-313.060423$ a.u.

0 imaginary frequencies 
Table S78. Methylpropane anion $\mathrm{n}=2$.

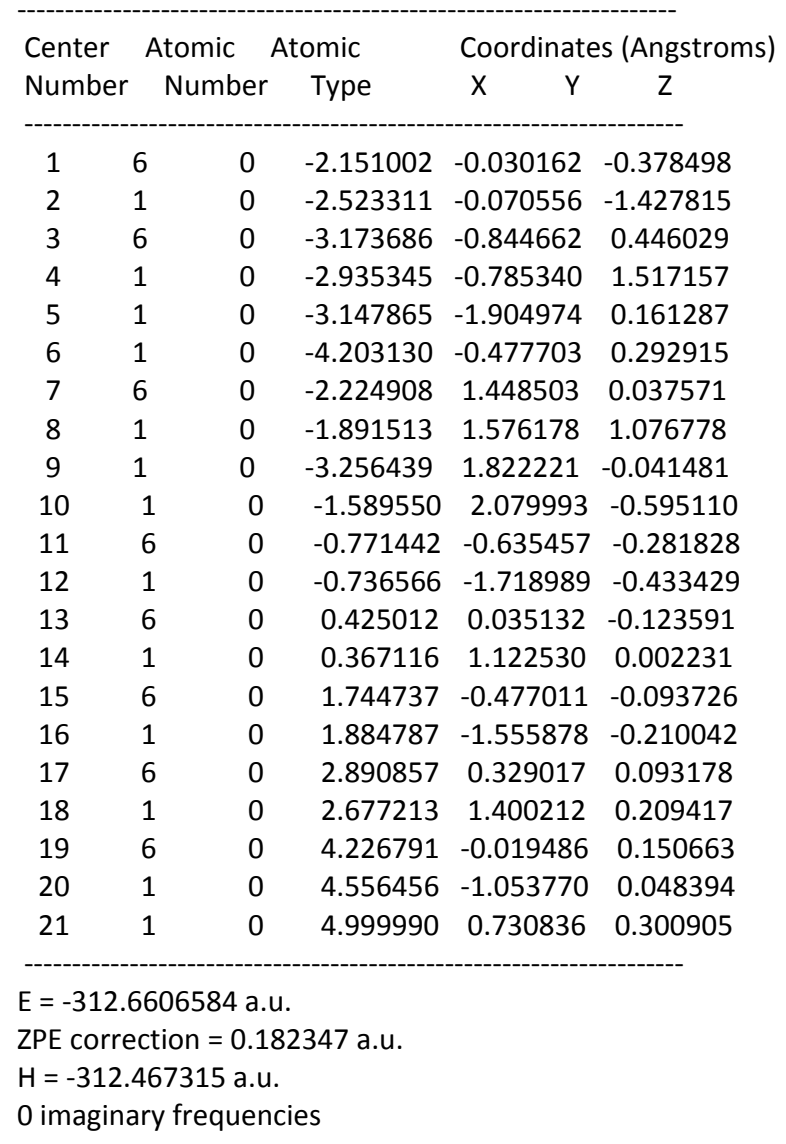


Table S79. Methylpropane neutral $n=3$.

\begin{tabular}{|c|c|c|c|c|c|}
\hline \multirow{2}{*}{$\begin{array}{l}\text { Center } \\
\text { Number }\end{array}$} & \multicolumn{2}{|c|}{ Atomic } & Atomic & \multicolumn{2}{|c|}{ Coordinates (Angstroms) } \\
\hline & & Number & Type & $X \quad Y$ & Z \\
\hline 1 & 6 & 0 & -3.401161 & -0.063917 & -0.337344 \\
\hline 2 & 1 & 0 & -3.836431 & -0.225743 & -1.338401 \\
\hline 3 & 6 & 0 & -4.221231 & -0.927537 & 0.646185 \\
\hline 4 & 1 & 0 & -3.854942 & -0.799220 & 1.672403 \\
\hline 5 & 1 & 0 & -4.151971 & -1.993346 & 0.396435 \\
\hline 6 & 1 & 0 & -5.280961 & -0.644448 & 0.623254 \\
\hline 7 & 6 & 0 & -3.541499 & 1.427638 & -0.007218 \\
\hline 8 & 1 & 0 & -3.149889 & 1.652850 & 0.993033 \\
\hline 9 & 1 & 0 & -4.597106 & 1.723223 & -0.024175 \\
\hline 10 & 1 & 0 & -3.006964 & 2.054635 & -0.730394 \\
\hline 11 & 6 & 0 & -1.975816 & -0.558561 & -0.398291 \\
\hline 12 & 1 & 0 & -1.870863 & -1.612964 & -0.666648 \\
\hline 13 & 6 & 0 & -0.850449 & 0.143437 & -0.152896 \\
\hline 14 & 1 & 0 & -0.918101 & 1.196969 & 0.118557 \\
\hline 15 & 6 & 0 & 0.487815 & -0.412419 & -0.227538 \\
\hline 16 & 1 & 0 & 0.567467 & -1.465913 & -0.502931 \\
\hline 17 & 6 & 0 & 1.623314 & 0.285466 & 0.018992 \\
\hline 18 & 1 & 0 & 1.543847 & 1.338768 & 0.295236 \\
\hline 19 & 6 & 0 & 2.961284 & -0.268858 & -0.056537 \\
\hline 20 & 1 & 0 & 3.037724 & -1.322325 & -0.334506 \\
\hline 21 & 6 & 0 & 4.092419 & 0.422656 & 0.189718 \\
\hline 22 & 1 & 0 & 4.009619 & 1.475551 & 0.467307 \\
\hline 23 & 6 & 0 & 5.481960 & -0.137915 & 0.114803 \\
\hline 24 & 1 & 0 & 6.000820 & -0.048393 & 1.079926 \\
\hline 25 & 1 & 0 & 6.092958 & 0.406768 & -0.619136 \\
\hline 26 & 1 & 0 & 5.474976 & -1.196352 & -0.169207 \\
\hline $\begin{array}{l}\text { ZPE cor } \\
\mathrm{H}=-39 \\
0 \text { imagi }\end{array}$ & .40 & 34375 a.l & 257 a.u. & & \\
\hline
\end{tabular}


Table S80. Methylpropane anion $\mathrm{n}=3$.

\begin{tabular}{|c|c|c|c|c|c|}
\hline \multirow{2}{*}{$\begin{array}{l}\text { Center } \\
\text { Number }\end{array}$} & \multirow{2}{*}{\multicolumn{2}{|c|}{$\begin{array}{l}\text { Atomic } \\
\text { Number }\end{array}$}} & \multirow{2}{*}{$\begin{array}{l}\text { Atomic } \\
\text { Type }\end{array}$} & \multicolumn{2}{|c|}{ Coordinates (Angstroms) } \\
\hline & & & & $X \quad Y$ & Z \\
\hline 1 & 6 & 0 & -3.326020 & 0.009190 & -0.335305 \\
\hline 2 & 1 & 0 & -3.781120 & -0.100528 & -1.342147 \\
\hline 3 & 6 & 0 & -4.297219 & -0.697191 & 0.638094 \\
\hline 4 & 1 & 0 & -3.957881 & -0.573417 & 1.675520 \\
\hline 5 & 1 & 0 & -4.341950 & -1.774655 & 0.432239 \\
\hline 6 & 1 & 0 & -5.319577 & -0.293575 & 0.553803 \\
\hline 7 & 6 & 0 & -3.298484 & 1.516166 & -0.035871 \\
\hline 8 & 1 & 0 & -2.879500 & 1.709508 & 0.960888 \\
\hline 9 & 1 & 0 & -4.314357 & 1.934891 & -0.064420 \\
\hline 10 & 1 & 0 & -2.687813 & 2.061939 & -0.765117 \\
\hline 11 & 6 & 0 & -1.967840 & -0.653718 & -0.324134 \\
\hline 12 & 1 & 0 & -1.989109 & -1.737624 & -0.472644 \\
\hline 13 & 6 & 0 & -0.746085 & -0.046004 & -0.184253 \\
\hline 14 & 1 & 0 & -0.738135 & 1.040210 & -0.048005 \\
\hline 15 & 6 & 0 & 0.546770 & -0.645843 & -0.192244 \\
\hline 16 & 1 & 0 & 0.606222 & -1.730138 & -0.319624 \\
\hline 17 & 6 & 0 & 1.735667 & 0.072322 & -0.040196 \\
\hline 18 & 1 & 0 & 1.619769 & 1.156681 & 0.086515 \\
\hline 19 & 6 & 0 & 3.054076 & -0.395478 & -0.026533 \\
\hline 20 & 1 & 0 & 3.227588 & -1.468204 & -0.148320 \\
\hline 21 & 6 & 0 & 4.188271 & 0.446450 & 0.137408 \\
\hline 22 & 1 & 0 & 3.953896 & 1.511547 & 0.254724 \\
\hline 23 & 6 & 0 & 5.520182 & 0.112907 & 0.168417 \\
\hline 24 & 1 & 0 & 5.855909 & -0.918474 & 0.061233 \\
\hline 25 & 1 & 0 & 6.290148 & 0.869036 & 0.303061 \\
\hline $\begin{array}{l}\mathrm{H}=-38 \\
0 \text { imag }\end{array}$ & כם. & $\begin{array}{l}8191 \text { a.u } \\
\text { y frequen }\end{array}$ & 171 a.u. & & \\
\hline
\end{tabular}


Table S81. Methylpropane neutral $n=4$.

\begin{tabular}{|c|c|c|c|c|c|}
\hline \multirow{2}{*}{$\begin{array}{l}\text { Center } \\
\text { Number }\end{array}$} & \multirow{2}{*}{\multicolumn{2}{|c|}{$\begin{array}{c}\text { Atomic } \\
\text { Number }\end{array}$}} & \multirow{2}{*}{$\begin{array}{l}\text { Atomic } \\
\text { Type }\end{array}$} & \multicolumn{2}{|c|}{ Coordinates (Angstroms) } \\
\hline & & & & $X \quad Y$ & Z \\
\hline 1 & 6 & 0 & -4.588086 & -0.055408 & -0.299622 \\
\hline 2 & 1 & 0 & -5.048614 & -0.258148 & -1.281706 \\
\hline 3 & 6 & 0 & -5.399442 & -0.856512 & 0.742692 \\
\hline 4 & 1 & 0 & -5.009584 & -0.683013 & 1.753412 \\
\hline 5 & 1 & 0 & -5.351122 & -1.934322 & 0.545238 \\
\hline 6 & 1 & 0 & -6.455051 & -0.558135 & 0.727249 \\
\hline 7 & 6 & 0 & -4.696937 & 1.452561 & -0.039550 \\
\hline 8 & 1 & 0 & -4.281304 & 1.719095 & 0.940610 \\
\hline 9 & 1 & 0 & -5.747675 & 1.765090 & -0.049677 \\
\hline 10 & 1 & 0 & -4.167367 & 2.034474 & -0.802899 \\
\hline 11 & 6 & 0 & -3.173134 & -0.576854 & -0.368225 \\
\hline 12 & 1 & 0 & -3.090553 & -1.642604 & -0.596232 \\
\hline 13 & 6 & 0 & -2.031048 & 0.115311 & -0.171694 \\
\hline 14 & 1 & 0 & -2.076895 & 1.179580 & 0.059065 \\
\hline 15 & 6 & 0 & -0.706011 & -0.465119 & -0.249975 \\
\hline 16 & 1 & 0 & -0.647497 & -1.529338 & -0.486195 \\
\hline 17 & 6 & 0 & 0.447781 & 0.224246 & -0.051576 \\
\hline 18 & 1 & 0 & 0.387810 & 1.288230 & 0.185783 \\
\hline 19 & 6 & 0 & 1.767966 & -0.350450 & -0.131495 \\
\hline 20 & 1 & 0 & 1.828346 & -1.413752 & -0.371660 \\
\hline 21 & 6 & 0 & 2.921576 & 0.338629 & 0.069267 \\
\hline 22 & 1 & 0 & 2.862808 & 1.401796 & 0.310015 \\
\hline 23 & 6 & 0 & 4.246447 & -0.239471 & -0.013331 \\
\hline 24 & 1 & 0 & 4.301034 & -1.303064 & -0.255375 \\
\hline 25 & 6 & 0 & 5.394085 & 0.441868 & 0.185673 \\
\hline 26 & 1 & 0 & 5.333559 & 1.505001 & 0.427325 \\
\hline 27 & 6 & 0 & 6.772024 & -0.144382 & 0.102509 \\
\hline 28 & 1 & 0 & 7.313248 & -0.027190 & 1.052257 \\
\hline 29 & 1 & 0 & 7.375462 & 0.362207 & -0.664262 \\
\hline 30 & 1 & 0 & 6.742073 & -1.212422 & -0.140981 \\
\hline$H=-46$ & .80 & 8954 a.u. & 69 a.u. & & \\
\hline
\end{tabular}


Table S82. Methylpropane anion $n=4$.

\begin{tabular}{|c|c|c|c|c|c|}
\hline \multirow{2}{*}{$\begin{array}{l}\text { Center } \\
\text { Number }\end{array}$} & \multirow{2}{*}{\multicolumn{2}{|c|}{$\begin{array}{l}\text { Atomic } \\
\text { Number }\end{array}$}} & Atomic & \multicolumn{2}{|c|}{ Coordinates (Angstroms) } \\
\hline & & & Type & $X \quad Y$ & Z \\
\hline 1 & 6 & 0 & -4.506544 & 0.080291 & -0.296766 \\
\hline 2 & 1 & 0 & -4.989225 & -0.067800 & -1.283773 \\
\hline 3 & 6 & 0 & -5.467060 & -0.545131 & 0.739639 \\
\hline 4 & 1 & 0 & -5.089943 & -0.385340 & 1.758691 \\
\hline 5 & 1 & 0 & -5.557317 & -1.628464 & 0.587030 \\
\hline 6 & 1 & 0 & -6.475721 & -0.108573 & 0.669332 \\
\hline 7 & 6 & 0 & -4.415496 & 1.597719 & -0.077099 \\
\hline 8 & 1 & 0 & -3.965161 & 1.826727 & 0.897971 \\
\hline 9 & 1 & 0 & -5.415197 & 2.052822 & -0.103375 \\
\hline 10 & 1 & 0 & -3.804523 & 2.081577 & -0.848448 \\
\hline 11 & 6 & 0 & -3.172298 & -0.631270 & -0.294341 \\
\hline 12 & 1 & 0 & -3.236283 & -1.718629 & -0.395972 \\
\hline 13 & 6 & 0 & -1.931040 & -0.067781 & -0.198813 \\
\hline 14 & 1 & 0 & -1.871414 & 1.020589 & -0.106206 \\
\hline 15 & 6 & 0 & -0.664947 & -0.733439 & -0.206000 \\
\hline 16 & 1 & 0 & -0.664570 & -1.823166 & -0.290014 \\
\hline 17 & 6 & 0 & 0.553412 & -0.074523 & -0.107557 \\
\hline 18 & 1 & 0 & 0.504229 & 1.017991 & -0.024485 \\
\hline 19 & 6 & 0 & 1.848482 & -0.626613 & -0.099565 \\
\hline 20 & 1 & 0 & 1.944692 & -1.712420 & -0.179880 \\
\hline 21 & 6 & 0 & 3.024956 & 0.135891 & 0.006168 \\
\hline 22 & 1 & 0 & 2.881787 & 1.220397 & 0.084946 \\
\hline 23 & 6 & 0 & 4.343141 & -0.306275 & 0.023267 \\
\hline 24 & 1 & 0 & 4.535849 & -1.379480 & -0.052967 \\
\hline 25 & 6 & 0 & 5.469233 & 0.562945 & 0.136066 \\
\hline 26 & 1 & 0 & 5.223617 & 1.628336 & 0.209950 \\
\hline 27 & 1 & 0 & 7.566999 & 0.999848 & 0.253799 \\
\hline 28 & 6 & 0 & 6.797687 & 0.236939 & 0.162993 \\
\hline 29 & 1 & 0 & 7.135021 & -0.796936 & 0.095454 \\
\hline $\begin{array}{l}H=-46 \\
0 \text { imagi }\end{array}$ & & $\begin{array}{l}04 \text { a.u. } \\
\text { frequenci }\end{array}$ & 043 a.u. & & \\
\hline
\end{tabular}


6. Cartesian coordinates and absolute energies of the delocalized and localized structures of acetone (1), acetone enolate anion, acetamide (2), acetamide enolate anion, acetic acid (3), acetic acid enolate anion, acetyl fluoride (4), acetyl fluoride enolate anion, methylpropane (5), and the methylpropyl anion, derived from the BLW method

Table S83. Delocalized $\mathrm{CH}_{3} \mathrm{COCH}_{3}$

\begin{tabular}{|c|c|c|c|c|c|}
\hline \multirow{2}{*}{$\begin{array}{l}\text { Atomic } \\
\text { Symbol }\end{array}$} & \multirow{2}{*}{\multicolumn{2}{|c|}{$\begin{array}{l}\text { Atomic } \\
\text { Number }\end{array}$}} & \multicolumn{3}{|c|}{ Coordinates (Angstroms) } \\
\hline & & & $x$ & $\mathrm{Y} \quad \mathrm{Z}$ & \\
\hline 0 & 8.0 & -0.00000 & 11224 & -1.3247205896 & 0.0000000000 \\
\hline C & 6.0 & 0.0000 & 10971 & -0.1051731871 & 0.0000000000 \\
\hline C & 6.0 & 1.2976 & 30979 & 0.6857899301 & 0.0000000000 \\
\hline $\mathrm{H}$ & 1.0 & 2.1467 & 455884 & -0.0012465670 & 0.0000000000 \\
\hline $\mathrm{H}$ & 1.0 & 1.3505 & 398085 & 1.3380565581 & -0.8820392583 \\
\hline $\mathrm{H}$ & 1.0 & 1.3505 & 398085 & 1.3380565581 & 0.8820392583 \\
\hline C & 6.0 & -1.2976 & 730539 & 0.6857900393 & 0.0000000000 \\
\hline $\mathrm{H}$ & 1.0 & -2.1467 & 455358 & -0.0012464226 & 0.0000000000 \\
\hline $\mathrm{H}$ & 1.0 & -1.3505 & 398442 & 1.3380559714 & 0.8820397314 \\
\hline $\mathrm{H}$ & 1.0 & -1.3505 & 398442 & 1.3380559714 & -0.8820397314 \\
\hline
\end{tabular}

$E=-193.0482662344$ a.u.

ZPE correction $=0.0827827$ a.u.

$\mathrm{H}=-192.9605801 \mathrm{a} . \mathrm{u}$.

0 imaginary frequencies

Table S84. Localized $\mathrm{CH}_{3} \mathrm{COCH}_{3}$

$\begin{array}{llll}\text { Atomic } & \text { Atomic } & & \text { Coordinates (Angstroms) } \\ \text { Symbol Number } & X & Y & Z\end{array}$

$\begin{array}{lllll}\text { O } & 8.0 & -0.0000008419 & -1.3301934182 & 0.0000000000\end{array}$

$\begin{array}{lllll}\text { C } & 6.0 & 0.0000009552 & -0.1207464045 & 0.0000000000\end{array}$

$\begin{array}{llllll}\text { C } & 6.0 & 1.3246986943 & 0.6984549050 & 0.0000000000\end{array}$

$\begin{array}{llllll}\mathrm{H} & 1.0 & 2.1656149075 & 0.0008006543 & 0.0000000000\end{array}$

H $\quad 1.0 \quad 1.3817551194 \quad 1.3359620340 \quad-0.8881073435$

H $\quad$\begin{tabular}{lllll}
\hline .0 & 1.3817551194 & 1.3359620340 & 0.8881073435
\end{tabular}

C $\quad 6.0-1.3246985812 \quad 0.6984546356 \quad 0.0000000000$

H $\quad \begin{array}{lllll}\mathrm{C} & 1.0 & -2.1656152692 & 0.0008011465 & 0.0000000000\end{array}$

$\begin{array}{lllll}\text { H } & 1.0 & -1.3817550518 & 1.3359613377 & 0.8881076565\end{array}$

H $\quad \begin{array}{lllll}1.0 & -1.3817550518 & 1.3359613377 & -0.8881076565\end{array}$

$E=-193.0307431604$ a.u.

ZPE correction $=0.0848296$ a.u.

$\mathrm{H}=-192.9411973$ a.u.

0 imaginary frequencies 
Table S85. Delocalized $\mathrm{CH}_{3} \mathrm{COCH}_{2}{ }^{-}$

\begin{tabular}{|c|c|c|c|c|c|}
\hline \multirow{2}{*}{$\begin{array}{l}\text { Atomic } \\
\text { Symbol }\end{array}$} & \multirow{2}{*}{\multicolumn{2}{|c|}{$\begin{array}{l}\text { Atomic } \\
\text { Number }\end{array}$}} & \multicolumn{3}{|c|}{ Coordinates (Angstroms) } \\
\hline & & & $\mathrm{x}$ & $\mathrm{Y} \quad \mathrm{Z}$ & \\
\hline $\mathrm{O}$ & 8.0 & -0.17358 & 68961 & 1.3290267932 & 0.0000000000 \\
\hline C & 6.0 & -0.10474 & 68437 & 0.0525114275 & 0.0000000000 \\
\hline C & 6.0 & 1.328 & 62943 & -0.5324324341 & 0.0000000000 \\
\hline $\mathrm{H}$ & 1.0 & 1.35452 & 65225 & -1.6324078703 & 0.0000000000 \\
\hline $\mathrm{H}$ & 1.0 & 1.87253 & 63248 & -0.1657415266 & -0.8831780222 \\
\hline $\mathrm{H}$ & 1.0 & 1.87253 & & -0.1657415266 & 0.8831780222 \\
\hline C & 6.0 & -1.15795 & 73180 & -0.8559098830 & 0.0000000000 \\
\hline $\mathrm{H}$ & 1.0 & -2.18935 & 501199 & -0.5041773903 & 0.0000000000 \\
\hline $\mathrm{H}$ & 1.0 & -0.98079 & 999231 & -1.9298796785 & 0.0000000000 \\
\hline
\end{tabular}

$E=-192.4506291738$ a.u.

ZPE correction $=0.07028777$ a.u.

$\mathrm{H}=-192.3753367$ a.u.

0 imaginary frequencies

Table S86. Localized $\mathrm{CH}_{3} \mathrm{COCH}_{2}{ }^{-}$

\begin{tabular}{|c|c|c|c|c|c|}
\hline \multirow{2}{*}{$\begin{array}{l}\text { Atomic } \\
\text { Symbol }\end{array}$} & \multirow{2}{*}{\multicolumn{2}{|c|}{$\begin{array}{l}\text { Atomic } \\
\text { Number }\end{array}$}} & \multicolumn{3}{|c|}{ Coordinates (Angstroms) } \\
\hline & & & $x$ & $Y \quad Z$ & \\
\hline $\mathrm{O}$ & 8.0 & -0.16053 & 08542 & 1.3099805667 & 0.0000000000 \\
\hline C & 6.0 & -0.07602 & 243431 & 0.0926281467 & 0.0000000000 \\
\hline C & 6.0 & 1.37609 & 89940 & -0.5168621122 & 0.0000000000 \\
\hline $\mathrm{H}$ & 1.0 & 1.36653 & 317097 & -1.6121980551 & 0.0000000000 \\
\hline $\mathrm{H}$ & 1.0 & 1.90655 & 534100 & -0.1599461457 & -0.8897361873 \\
\hline $\mathrm{H}$ & 1.0 & 1.90655 & 53100 & -0.1599461457 & 0.8897361873 \\
\hline C & 6.0 & -1.23010 & 70117 & -0.8963490043 & 0.0000000000 \\
\hline $\mathrm{H}$ & 1.0 & -2.23953 & 337108 & -0.5011923606 & 0.0000000000 \\
\hline $\mathrm{H}$ & 1.0 & -1.02833 & 372381 & -1.9608669786 & 0.0000000000 \\
\hline
\end{tabular}

$E=-192.3680199341$ a.u.

ZPE correction $=0.0748781$ a.u.

$\mathrm{H}=-192.2885617$ a.u.

0 imaginary frequencies 
Table S87. Delocalized $\mathrm{NH}_{2} \mathrm{COCH}_{3}$

\begin{tabular}{|c|c|c|c|c|c|}
\hline \multirow{2}{*}{$\begin{array}{l}\text { Atomic } \\
\text { Symbol }\end{array}$} & \multirow{2}{*}{\multicolumn{2}{|c|}{$\begin{array}{l}\text { Atomic } \\
\text { Number }\end{array}$}} & \multicolumn{3}{|c|}{ Coordinates (Angstroms) } \\
\hline & & & $\mathrm{x}$ & $\mathrm{Y} \quad \mathrm{Z}$ & \\
\hline $\mathrm{O}$ & 8.0 & -0.20245 & 99512 & 1.3072186708 & 0.0000000000 \\
\hline C & 6.0 & -0.02021 & 30691 & 0.0953589828 & 0.0000000000 \\
\hline C & 6.0 & 1.37410 & 63747 & -0.5101491495 & 0.0000000000 \\
\hline $\mathrm{H}$ & 1.0 & 1.38539 & 03207 & -1.6054278635 & 0.0000000000 \\
\hline $\mathrm{H}$ & 1.0 & 1.91288 & 392226 & -0.1492151160 & -0.8824959671 \\
\hline $\mathrm{H}$ & 1.0 & 1.91288 & 392226 & -0.1492151160 & 0.8824959671 \\
\hline N & 7.0 & -1.06296 & 543933 & -0.7928432655 & 0.0000000000 \\
\hline $\mathrm{H}$ & 1.0 & -2.00583 & 356153 & -0.4262727180 & 0.0000000000 \\
\hline $\mathrm{H}$ & 1.0 & -0.92964 & 114533 & -1.7928999780 & 0.0000000000 \\
\hline
\end{tabular}

$E=-209.1089281413$ a.u.

ZPE correction $=0.0739212$ a.u.

$\mathrm{H}=-209.0296596$ a.u.

0 imaginary frequencies

Table S88. Localized $\mathrm{NH}_{2} \mathrm{COCH}_{3}$

\begin{tabular}{|c|c|c|c|c|c|}
\hline \multirow{2}{*}{$\begin{array}{l}\text { Atomic } \\
\text { Symbol }\end{array}$} & \multirow{2}{*}{\multicolumn{2}{|c|}{$\begin{array}{l}\text { Atomic } \\
\text { Number }\end{array}$}} & \multicolumn{3}{|c|}{ Coordinates (Angstroms) } \\
\hline & & & $x$ & $\mathrm{Y} \quad \mathrm{Z}$ & \\
\hline $\mathrm{O}$ & 8.0 & -0.19842 & 284921 & 1.3134499007 & 0.0000000000 \\
\hline $\mathrm{C}$ & 6.0 & -0.00349 & 950813 & 0.1307579819 & 0.0000000000 \\
\hline C & 6.0 & 1.41307 & 780499 & -0.4966619319 & 0.0000000000 \\
\hline $\mathrm{H}$ & 1.0 & 01.38895 & 520490 & -1.5900082468 & 0.0000000000 \\
\hline $\mathrm{H}$ & 1.0 & $0 \quad 1.94799$ & 920625 & -0.1536380866 & -0.8887284611 \\
\hline $\mathrm{H}$ & 1.0 & $0 \quad 1.94799$ & 920625 & -0.1536380866 & 0.8887284611 \\
\hline $\mathrm{N}$ & 7.0 & $0-1.11361$ & 168060 & -0.8176232614 & 0.0000000000 \\
\hline $\mathrm{H}$ & 1.0 & $0-2.04883$ & 317451 & -0.4437212802 & 0.0000000000 \\
\hline $\mathrm{H}$ & 1.0 & $0-0.96948$ & 814411 & -1.8123625421 & 0.0000000000 \\
\hline \multicolumn{6}{|c|}{$\begin{array}{l}E=-209.0588795619 \text { a.u. } \\
\text { ZPE correction }=0.079381 \text { a.u. } \\
H=-208.9749926 \text { a.u. } \\
0 \text { imaginary frequencies }\end{array}$} \\
\hline
\end{tabular}


Table S89. Delocalized $\mathrm{NH}_{2} \mathrm{COCH}_{2}{ }^{-}$

\begin{tabular}{|c|c|c|c|c|c|}
\hline \multirow{2}{*}{$\begin{array}{l}\text { Atomic } \\
\text { Symbol }\end{array}$} & \multirow{2}{*}{\multicolumn{2}{|c|}{$\begin{array}{l}\text { Atomic } \\
\text { Number }\end{array}$}} & \multicolumn{3}{|c|}{ Coordinates (Angstroms) } \\
\hline & & & $x$ & $\mathrm{Y} \quad \mathrm{Z}$ & \\
\hline 0 & 8.0 & -0.12524 & 21666 & 1.3261363714 & 0.0000000000 \\
\hline C & 6.0 & 0.09892 & 263382 & 0.0750340688 & 0.0000000000 \\
\hline C & 6.0 & 1.32888 & 330390 & -0.5895920370 & 0.0000000000 \\
\hline $\mathrm{H}$ & 1.0 & 2.24958 & 808732 & -0.0137778769 & 0.0000000000 \\
\hline $\mathrm{H}$ & 1.0 & 1.39864 & 455847 & -1.6769274715 & 0.0000000000 \\
\hline $\mathrm{N}$ & 7.0 & -1.07645 & 580811 & -0.7450727239 & 0.0000000000 \\
\hline $\mathrm{H}$ & 1.0 & -1.97242 & 292135 & -0.2838493499 & 0.0000000000 \\
\hline $\mathrm{H}$ & 1.0 & -1.03534 & 434149 & -1.7536209996 & 0.0000000000 \\
\hline
\end{tabular}

$E=-208.4919017363$ a.u.

ZPE correction $=0.0581998$ a.u.

$\mathrm{H}=-208.4282587$ a.u.

0 imaginary frequencies

Table S90. Localized $\mathrm{NH}_{2} \mathrm{COCH}_{2}{ }^{-}$

\begin{tabular}{|c|c|c|c|c|c|}
\hline \multirow{2}{*}{$\begin{array}{l}\text { Atomic } \\
\text { Symbol }\end{array}$} & \multirow{2}{*}{\multicolumn{2}{|c|}{$\begin{array}{l}\text { Atomic } \\
\text { Number }\end{array}$}} & \multicolumn{3}{|c|}{ Coordinates (Angstroms) } \\
\hline & & & $x$ & $\mathrm{Y} \quad \mathrm{Z}$ & \\
\hline $\mathrm{O}$ & 8.0 & -0.12044 & 81132 & 1.3289919415 & 0.0000000000 \\
\hline C & 6.0 & 0.07123 & 70538 & 0.1337227785 & 0.0000000000 \\
\hline C & 6.0 & 1.39066 & 24438 & -0.6117498192 & 0.0000000000 \\
\hline $\mathrm{H}$ & 1.0 & 2.29748 & 336365 & -0.0210003473 & 0.0000000000 \\
\hline $\mathrm{H}$ & 1.0 & 1.40969 & 973881 & -1.6949383954 & 0.0000000000 \\
\hline $\mathrm{N}$ & 7.0 & -1.12589 & 998592 & -0.7408433582 & 0.0000000000 \\
\hline $\mathrm{H}$ & 1.0 & -2.03811 & 125410 & -0.3154924816 & 0.0000000000 \\
\hline $\mathrm{H}$ & 1.0 & -1.01805 & 570496 & -1.7403603369 & 0.0000000000 \\
\hline
\end{tabular}

$E=-208.4001697985$ a.u.

ZPE correction $=0.0681611$ a.u.

$\mathrm{H}=-208.3274893$ a.u.

0 imaginary frequencies

Table S91. Delocalized $\mathrm{CH}_{3} \mathrm{COOH}$

$\begin{array}{llll}\text { Atomic } & \text { Atomic } & & \text { Coordinates (Angstroms) } \\ \text { Symbol Number } & \text { X } & \text { Y } & \text { Z }\end{array}$

$\begin{array}{llllll}\text { O } & 8.0 & -0.2552745401 & 1.3150770461 & 0.0000000000\end{array}$

$\begin{array}{llllll}\text { C } & 6.0 & 0.0159775747 & 0.1330442565 & 0.0000000000\end{array}$

$\begin{array}{llllll}\text { C } & 6.0 & 1.3990174644 & -0.4638993984 & 0.0000000000\end{array}$

$\begin{array}{lllll}\mathrm{H} & 1.0 & 2.1463919862 & 0.3312207724 & 0.0000000000\end{array}$

$\begin{array}{llllll}\text { H } & 1.0 & 1.5313474846 & -1.0999725098 & -0.8825382396\end{array}$

$\begin{array}{llllll}\text { H } & 1.0 & 1.5313474846 & -1.0999725098 & 0.8825382396\end{array}$

$\begin{array}{llllll}0 & 8.0 & -0.9447125233 & -0.8308595388 & 0.0000000000\end{array}$

H $\quad \begin{array}{lllll}1.0 & -1.8064120186 & -0.3721677582 & 0.0000000000\end{array}$

$E=-228.9774346276$ a.u.

ZPE correction $=0.0617808$ a.u.

$\mathrm{H}=-228.9109629$ a.u.

0 imaginary frequencies 
Table S92. Localized $\mathrm{CH}_{3} \mathrm{COOH}$

\begin{tabular}{|c|c|c|c|c|c|}
\hline \multirow{2}{*}{$\begin{array}{l}\text { Atomic } \\
\text { Symbol }\end{array}$} & \multirow{2}{*}{\multicolumn{2}{|c|}{$\begin{array}{l}\text { Atomic } \\
\text { Number }\end{array}$}} & \multicolumn{3}{|c|}{ Coordinates (Angstroms) } \\
\hline & & & $x$ & $\mathrm{Y} \quad \mathrm{Z}$ & \\
\hline 0 & 8.0 & -0.24814 & 26995 & 1.3343312710 & 0.0000000000 \\
\hline C & 6.0 & 0.02686 & 07627 & 0.1752071034 & 0.0000000000 \\
\hline C & 6.0 & 1.42262 & 93904 & -0.4697052294 & 0.0000000000 \\
\hline $\mathrm{H}$ & 1.0 & 2.17956 & 537743 & 0.3174890906 & 0.0000000000 \\
\hline $\mathrm{H}$ & 1.0 & 1.53630 & 990359 & -1.0949052780 & -0.8881005303 \\
\hline $\mathrm{H}$ & 1.0 & 1.53630 & 990359 & -1.0949052780 & 0.8881005303 \\
\hline $\mathrm{O}$ & 8.0 & -0.98904 & 423164 & -0.8567347360 & 0.0000000000 \\
\hline $\mathrm{H}$ & 1.0 & -1.84680 & 040708 & -0.3983065837 & 0.0000000000 \\
\hline
\end{tabular}

$E=-228.9368684139$ a.u.

ZPE correction $=0.06502528$ a.u.

$\mathrm{H}=-228.8673545$ a.u.

0 imaginary frequencies

Table S93. Delocalized ${ }^{-} \mathrm{CH}_{2} \mathrm{COOH}$

\begin{tabular}{|c|c|c|c|c|c|}
\hline \multirow{2}{*}{$\begin{array}{l}\text { Atomic } \\
\text { Symbol }\end{array}$} & \multirow{2}{*}{\multicolumn{2}{|c|}{$\begin{array}{l}\text { Atomic } \\
\text { Number }\end{array}$}} & \multicolumn{3}{|c|}{ Coordinates (Angstroms) } \\
\hline & & & $\mathrm{x}$ & $\mathrm{Y} \quad \mathrm{Z}$ & \\
\hline 0 & 8.0 & -0.17425 & 26183 & 1.3232442217 & 0.0000000000 \\
\hline C & 6.0 & 0.08613 & 58220 & 0.0858495819 & 0.0000000000 \\
\hline C & 6.0 & 1.28907 & 13025 & -0.5943981871 & 0.0000000000 \\
\hline $\mathrm{H}$ & 1.0 & 2.21918 & 397598 & -0.0338801337 & 0.0000000000 \\
\hline $\mathrm{H}$ & 1.0 & 1.32530 & 664489 & -1.6797019597 & 0.0000000000 \\
\hline $\mathrm{O}$ & 8.0 & -1.07659 & 914373 & -0.7613876522 & 0.0000000000 \\
\hline $\mathrm{H}$ & 1.0 & -1.79007 & 738537 & -0.1011503053 & 0.0000000000 \\
\hline
\end{tabular}

$E=-228.3771789232$ a.u.

ZPE correction $=0.0475105$ a.u.

$\mathrm{H}=-228.3245128$ a.u.

0 imaginary frequencies

Table S94. Localized ${ }^{-} \mathrm{CH}_{2} \mathrm{COOH}$

$\begin{array}{llll}\text { Atomic } & \text { Atomic } & & \text { Coordinates (Angstroms) } \\ \text { Symbol } & \text { Number } & \text { X } & \text { Y }\end{array}$

$\begin{array}{llllll}0 & 8.0 & -0.1672816718 & 1.3198349910 & 0.0000000000\end{array}$

$\begin{array}{llllll}\text { C } & \quad 6.0 & 0.0608844053 & 0.1390365284 & 0.0000000000\end{array}$

$\begin{array}{llllll}\text { C } & 6.0 & 1.3613473284 & -0.6179165238 & 0.0000000000\end{array}$

$\begin{array}{llllll}\mathrm{H} & 1.0 & 2.2706733267 & -0.0321183778 & 0.0000000000\end{array}$

$\begin{array}{llllll}\text { H } & 1.0 & 1.3454073827 & -1.6975232427 & 0.0000000000\end{array}$

$\begin{array}{llllll}0 & 8.0 & -1.1272187235 & -0.7523807037 & 0.0000000000\end{array}$

H $\quad 1.0 \quad-1.8650266239-0.12035710590 .0000000000$

$E=-228.2818329505$ a.u.

ZPE correction $=0.0538732$ a.u.

$\mathrm{H}=-228.2234313$ a.u.

0 imaginary frequencies 
Table S95. Delocalized $\mathrm{CH}_{3} \mathrm{COF}$

\begin{tabular}{llllll} 
Atomic & Atomic & \multicolumn{4}{c}{ Coordinates (Angstroms) } \\
Symbol & Number & X & Y & Z & \\
--------------- & \\
O & 8.0 & -0.0932064900 & 1.3738432516 & 0.0001233650 \\
C & 6.0 & 0.0001269161 & 0.1872410742 & 0.0000076618 \\
C & 6.0 & -1.0756776915 & -0.8559676739 & -0.0000100733 \\
H & 1.0 & -2.0532944858 & -0.3722245499 & -0.0000920380 \\
H & 1.0 & -0.9700357180 & -1.4956843086 & 0.8832601487 \\
H & 1.0 & -0.9699355065 & -1.4958205770 & -0.8831645864 \\
F & 9.0 & 1.2445929756 & -0.4013612163 & -0.0001244778
\end{tabular}

$E=-252.9905148632$ a.u.

ZPE correction $=0.04863376$ a.u.

$\mathrm{H}=-252.9372814$ a.u.

0 imaginary frequencies

Table S96. Localized $\mathrm{CH}_{3} \mathrm{COF}$

\begin{tabular}{llll} 
Atomic & Atomic & & \multicolumn{3}{c}{ Coordinates (Angstroms) } \\
Symbol Number & X & Y & Z
\end{tabular}

$\begin{array}{llllll}0 & 8.0 & 0.0840608994 & 1.3536887290 & 0.0001847457\end{array}$

$\begin{array}{llllll}\text { C } & 6.0 & 0.1156857807 & 0.1784996829 & 0.0000420780\end{array}$

$\begin{array}{llllll}\text { C } & 6.0 & 1.2905217972 & -0.8069391304 & 0.0000973967\end{array}$

$\begin{array}{llllll}\text { H } & 1.0 & 2.2259757457 & -0.2430090867 & -0.0002831174\end{array}$

H $\quad \begin{array}{llllll}1.0 & 1.2364039771 & -1.4375560777 & -0.8892604789\end{array}$

$\begin{array}{llllll}\text { H } & 1.0 & 1.2365383399 & -1.4372409711 & 0.8896022405\end{array}$

F $\quad 9.0-1.1252534911-0.5631304572-0.0003828646$

$E=-252.9603672697$ a.u.

ZPE correction $=0.05013458$ a.u.

$\mathrm{H}=-252.9057266$ a.u.

0 imaginary frequencies

Table S97. Delocalized ${ }^{-} \mathrm{CH}_{2} \mathrm{COF}$

\begin{tabular}{|c|c|c|c|}
\hline \multirow{2}{*}{$\begin{array}{l}\text { Atomic } \\
\text { Symbol }\end{array}$} & \multirow{2}{*}{$\begin{array}{l}\text { Atomic } \\
\text { Number }\end{array}$} & \multicolumn{2}{|c|}{ Coordinates (Angstroms) } \\
\hline & & $\mathrm{Y} \quad \mathrm{Z}$ & \\
\hline $\mathrm{O}$ & $8.0-0.8310486129$ & 1.0929075841 & 0.0000000000 \\
\hline $\mathrm{C}$ & $6.0-0.0008721058$ & 0.1815599815 & 0.0000000000 \\
\hline C & 6.01 .3732260049 & 0.0958996424 & 0.0000000000 \\
\hline $\mathrm{H}$ & 1.01 .9611252791 & 1.0089470193 & 0.0000000000 \\
\hline $\mathrm{H}$ & 1.01 .8766797202 & -0.8640916184 & 0.0000000000 \\
\hline $\mathrm{F}$ & $9.0-0.6024132855$ & -1.1722716090 & 0.0000000000 \\
\hline \multicolumn{4}{|c|}{ FINAL R-B3LYP ENERGY IS $\quad-252.4088499539$} \\
\hline \multicolumn{4}{|c|}{ ZPE Correction: 21.845188 KCAL/MOL } \\
\hline \multicolumn{4}{|c|}{ Enthalpy: add $24.953 \mathrm{kcal} / \mathrm{mol}$ to the above electronic energy } \\
\hline \multicolumn{4}{|c|}{$E=-252.4088499539$ a.u. } \\
\hline \multicolumn{4}{|c|}{ ZPE correction $=0.0348130 \mathrm{a} . \mathrm{u}}$. \\
\hline \multicolumn{4}{|c|}{$H=-252.3691338$ a.u. } \\
\hline \multicolumn{4}{|c|}{0 imaginary frequencies } \\
\hline
\end{tabular}


Table S98. Localized ${ }^{-} \mathrm{CH}_{2} \mathrm{COF}$

$\begin{array}{llll}\text { Atomic } & \text { Atomic } & & \text { Coordinates (Angstroms) } \\ \text { Symbol } & \text { Number } & \text { X } & \text { Y } \\ \text { Z }\end{array}$

$\begin{array}{llllll}\text { O } & 8.0 & -0.8464677955 & 1.0703100630 & 0.0000000000\end{array}$

$\begin{array}{llllll}\text { C } & 6.0 & -0.0547320398 & 0.1925941851 & 0.0000000000\end{array}$

$\begin{array}{llllll}\text { C } & 6.0 & 1.4436295563 & 0.1021959881 & 0.0000000000\end{array}$

H $\quad \begin{array}{lllll}1.0 & 1.9937556735 & 1.0331841023 & 0.0000000000\end{array}$

H $\quad \begin{array}{lllll}\text { H } & 1.0 & 1.9012107979 & -0.8738152402 & 0.0000000000\end{array}$

F $\quad 9.0 \quad-0.6606991924-1.18151809840 .0000000000$

FINAL R-B3LYP ENERGY IS $\quad-252.3179971121$

ZPE Correction: $24.570474 \mathrm{KCAL} / \mathrm{MOL}$

Enthalpy: add $27.418 \mathrm{kcal} / \mathrm{mol}$ to the above electronic energy

$E=-252.3179971121$ a.u.

ZPE correction $=0.03915613$ a.u.

$\mathrm{H}=-252.2743031$ a.u.

0 imaginary frequencies

Table S99. Delocalized $\mathrm{H}_{3} \mathrm{CCH}\left(\mathrm{CH}_{3}\right)_{2}$

$\begin{array}{llll}\text { Atomic } & \text { Atomic } & & \text { Coordinates (Angstroms) } \\ \text { Symbol Number } & \text { X } & \text { Y Z Z }\end{array}$

C $\quad \begin{array}{lllll}6.0 & -0.0000005015 & 1.4605407530 & -0.0930658400\end{array}$

H $\quad \begin{array}{llllll}\text { H } & 1.0 & -0.0000056793 & 1.5076191211 & -1.1918334818\end{array}$

$\begin{array}{llllll}H & 1.0 & 0.8868745102 & 2.0024542806 & 0.2603932996\end{array}$

$\begin{array}{lllll}\text { H } & 1.0 & -0.8868691456 & 2.0024579352 & 0.2604032593\end{array}$

$\begin{array}{lllll}\text { C } & 6.0 & -0.0000000992 & 0.0000004547 & 0.3827315966\end{array}$

$\begin{array}{llllll}\text { H } & 1.0 & 0.0000000364 & 0.0000035212 & 1.4847949946\end{array}$

C $\quad 6.0-1.2648632038-0.7302729549-0.0930673474$

H $\quad 1.0-1.3056344125-0.7538104301-1.1918360211$

H $\quad \begin{array}{lllll}1.0 & -2.1776101279 & -0.2331727209 & 0.2603977102\end{array}$

H $\quad \begin{array}{lllll}1.0 & -1.2907394229 & -1.7692842675 & 0.2603990079\end{array}$

$\begin{array}{llllll}\text { C } & 6.0 & 1.2648637492 & -0.7302731351 & -0.0930668965\end{array}$

H $\quad \begin{array}{lllll}\text { H } & 1.0 & 1.3056374076 & -0.7538063050 & -1.1918351577\end{array}$

H $\quad \begin{array}{llllll}1.0 & 1.2907380437 & -1.7692843116 & 0.2603991862\end{array}$

$\begin{array}{lllll}H & 1.0 & 2.1776088452 & -0.2331729407 & 0.2604026902\end{array}$

$E=-158.3406036466$ a.u.

ZPE correction $=0.13256790$ a.u.

$H=-158.2015438$ a.u.

0 imaginary frequencies 
Table S100. Delocalized ${ }^{-} \mathrm{H}_{2} \mathrm{CCH}\left(\mathrm{CH}_{3}\right)_{2}$

\begin{tabular}{lrrrrr} 
Atomic & Atomic & \multicolumn{3}{c}{ Coordinates (Angstroms) } \\
Symbol & Number & X & Y & Z & \\
- & & & & \\
C & 6.0 & 0.0277209921 & 0.0879315817 & -0.3697774001 \\
H & 1.0 & 0.0412799804 & 0.0293056939 & -1.4770296173 \\
C & 6.0 & 0.8897386195 & -1.1251830186 & 0.1189038528 \\
H & 1.0 & 0.9036275421 & -1.1573365797 & 1.2203013008 \\
H & 1.0 & 1.9284269541 & -1.0073308274 & -0.2131886915 \\
H & 1.0 & 0.5150184107 & -2.1055963884 & -0.2507875236 \\
C & 6.0 & -1.4312115149 & -0.1565284941 & 0.0720140926 \\
H & 1.0 & -1.4985037951 & -0.1075444321 & 1.1709851495 \\
H & 1.0 & -1.8109332776 & -1.1443044436 & -0.2397653054 \\
H & 1.0 & -2.0824656260 & 0.6272165813 & -0.3320942442 \\
C & 6.0 & 0.5112916119 & 1.4650216080 & 0.0476758485 \\
H & 1.0 & 1.5425325999 & 1.6596145001 & -0.2980390958 \\
H & 1.0 & 0.4650325029 & 1.5910212187 & 1.1467656337
\end{tabular}

$E=-157.6663124694$ a.u.

ZPE correction $=0.11584208$ a.u.

$\mathrm{H}=-157.5441308$ a.u.

0 imaginary frequencies 